\title{
Extended ionized gas in elliptical galaxies ${ }^{\star}$
}

\section{Velocity and monochromatic maps of 11 elliptical and lenticular galaxies}

\author{
H. Plana ${ }^{1,2}$, J. Boulesteix ${ }^{1}$, Ph. Amram ${ }^{1}$, C. Carignan ${ }^{3}$, and C. Mendes de Oliveira ${ }^{2}$ \\ ${ }^{1}$ Observatoire de Marseille, 2 Place Le Verrier, F-13248 Marseille Cedex 04, France \\ ${ }^{2}$ Instituto Astronômico e Geofísico (IAG), Av. Miguel Stefano 4200 CEP: 04301-904 São Paulo, Brazil \\ ${ }^{3}$ Dept. de Physique, Université de Montréal CP 6128, Succ. Centre ville Montréal, P.GC Canada, H3C-3J7, Canada
}

Received March 11; accepted June 16, 1997

\begin{abstract}
For the last ten years faint ionized gas detection has been carried out for elliptical galaxies with success. The kinematics is essential to understand galaxy gas origin and fate. Here we present a sample of 11 elliptical and lenticular galaxies observed with the "Cigale" scanning Perot-Fabry instrument. For each galaxy monochromatic and velocity map of ionized gas is presented. Geometrical properties such as viewing angles and axis ratios are also derived from observations. Double gaseous components are found in 3 galaxies of our sample, implying an external origin for at least part of the observed gas.
\end{abstract}

Key words: galaxies: elliptical and lenticular, kinematics, ISM

\section{Introduction}

Elliptical galaxies were usually known to be gas free, but in the past ten years several surveys have been done using CCD subtraction techniques in order to detect extended gaseous disks (Demoulin et al. 1984; Kim 1989; Buson et al. 1993; Goudfrooij et al. 1994; Macchetto et al. 1996).

In the same way multi-phase Interstellar Medium (ISM) has been discovered in early type galaxies:

- A hot component emitting in X-rays (Forman et al. 1985; Fabbiano et al. 1992),

- A cold component atomic gas (Knapp et al. 1985; van Gorkom et al. 1986) and molecular gas (Lees et al. 1991; Braine et al. 1994),

Send offprint requests to: $\mathrm{H}$. Plana

* Based on observations collected with the S.A.O. $6 \mathrm{~m}$ telescope located in Nizhnij Arkhyz (Russia), the $3.6 \mathrm{~m}$ CFH telescope and the $3.6 \mathrm{~m}$ telescope at ESO.
- A cold dust component, frequently detected but his role is not well understood (Goudfrooij et al. 1994; Kim 1989).

The ionized gas is a good tracer of potential perturbation, it responds quickly to gravitational variation. It can be located in two preferred planes, one perpendicular to the shortest axis and the other one perpendicular to the longest axis (Tholine 1982; Heiligman et al. 1979). It has been shown that gaseous disks can be used to constrain the true shape of the ellipsoid (de Zeeuw \& Franx 1989; Bertola et al. 1991). The 2D velocity field of ionized gas is an important tool to determine viewing angles and axis ratios from observational parameters. Table 1 shows us main characteristics of the galaxy sample. It consists in 11 elliptical and lenticular galaxies: there are nearby (the maximum redshift is $4800 \mathrm{~km} \mathrm{~s}^{-1}$ ) in order to have sufficient spatial resolution, there are field and cluster objects in order to see environment influence and all objects have been chosen to have a multiphase ISM (see Table 5).

\section{Observations}

Observations were carried out with the Cigale instrument (Boulesteix et al. 1983; Amram et al. 1991) at the $6 \mathrm{~m}$ telescope (SAO Russia) between 1991 and 1994, the $3.6 \mathrm{~m}$ telescope at ESO in 1990 and 1995 and the $3.6 \mathrm{~m}$ telescope at $\mathrm{CFH}$ in Hawaii in 1995. IPCS detector was used at the SAO $6 \mathrm{~m}$ and ESO $3.6 \mathrm{~m}$ telescopes.

For the $6 \mathrm{~m}$ telescope observations, CIGALE was attached at the F/4 prime focus and mainly consists of a scanning Perot-Fabry interferometer with a focal reducer, followed by a magnetically focussed Image Photon Counting System (IPCS), giving $512 \times 512$ pixels of $30 \mu \mathrm{m}$ on a side (Afanasiev et al. 1987). The final aperture ratio is $\mathrm{f} / 2.43$ and the pixel sizes are given in Table 3 . The IPCS, with a time resolution of $1 / 50 \mathrm{~s}$ and zero readout noise enables one to scan the interferometer rapidly (typically 10 to 20 s per channel), avoiding sky transparency and seeing 
Table 1. Galaxies main characteristics

\begin{tabular}{|c|c|c|c|c|c|c|}
\hline \multirow[t]{2}{*}{ Name } & \multicolumn{2}{|c|}{ Coordinates (1950) } & \multirow{2}{*}{$\begin{array}{c}\text { Morphological } \\
\text { Type (RSA/RC3) }\end{array}$} & \multirow{2}{*}{$\begin{array}{c}\text { Systemic } \\
\text { Velocity }\left(\mathrm{km} \mathrm{s}^{-1}\right) \\
\end{array}$} & \multirow[t]{2}{*}{$B_{\mathrm{T}}$} & \multirow[t]{2}{*}{ Environment } \\
\hline & $\alpha$ & $\delta$ & & & & \\
\hline NGC 404 & $01^{\mathrm{h}} 06^{\mathrm{m}} 39.2^{\mathrm{s}}$ & $+35^{\circ} 27^{\prime} 06^{\prime \prime}$ & $\mathrm{SO} / \mathrm{SO}$ & -36 & 10.96 & Isolated \\
\hline NGC 708 & $01^{\mathrm{h}} 49^{\mathrm{m}} 50.0^{\mathrm{s}}$ & $+35^{\circ} 54^{\prime} 20^{\prime \prime}$ & $\mathrm{E} 2 / \mathrm{E}$ & 4827 & 13.8 & Cluster \\
\hline NGC 1052 & $02^{\mathrm{h}} 38^{\mathrm{m}} 37.4^{\mathrm{s}}$ & $-08^{\circ} 28^{\prime} 10^{\prime \prime}$ & $\mathrm{E} 3 / \mathrm{E} 4$ & 1470 & 11.53 & Companions \\
\hline NGC 2974 & $09^{\mathrm{h}} 40^{\mathrm{m}} 01.9^{\mathrm{s}}$ & $-03^{\circ} 28^{\prime} 15^{\prime \prime}$ & $\mathrm{E} 4 / \mathrm{E} 4$ & 1924 & 11.68 & Isolated \\
\hline NGC 4546 & $12^{\mathrm{h}} 32^{\mathrm{m}} 55.0^{\mathrm{s}}$ & $-03^{\circ} 31^{\prime} 06^{\prime \prime}$ & SB0 & 1050 & 11.3 & Isolated \\
\hline NGC 5846 & $15^{\mathrm{h}} 03^{\mathrm{m}} 57.0^{\mathrm{s}}$ & $+01^{\circ} 47^{\prime} 57^{\prime \prime}$ & $\mathrm{SO} / \mathrm{E} 0$ & 1709 & 11.1 & Cluster \\
\hline NGC 5866 & $15^{\mathrm{h}} 05^{\mathrm{m}} 07.0^{\mathrm{s}}$ & $+55^{\circ} 57^{\prime} 16^{\prime \prime}$ & $\mathrm{SO}$ & 769 & 10.8 & Companions \\
\hline NGC 5898 & $15^{\mathrm{h}} 15^{\mathrm{m}} 17.0^{\mathrm{s}}$ & $-23^{\circ} 54^{\prime} 54^{\prime \prime}$ & $\mathrm{SO} / \mathrm{E} 0$ & 2103 & 10.8 & Companions \\
\hline NGC 6868 & $20^{\mathrm{h}} 06^{\mathrm{m}} 16.0^{\mathrm{s}}$ & $-48^{\circ} 31^{\prime} 36^{\prime \prime}$ & E3/E2 & 2876 & 11.6 & Cluster \\
\hline NGC 7014 & $21^{\mathrm{h}} 04^{\mathrm{m}} 29.0^{\mathrm{s}}$ & $-47^{\circ} 22^{\prime} 48^{\prime \prime}$ & $\mathrm{SO}$ & 4750 & 13.38 & Companions \\
\hline NGC 7332 & $22^{\mathrm{h}} 35^{\mathrm{m}} 00.1^{\mathrm{s}}$ & $+23^{\circ} 32^{\prime} 16^{\prime \prime}$ & $\mathrm{SO} / \mathrm{L}$ & 1300 & 11.58 & Companions \\
\hline
\end{tabular}

variation problems during long exposures.

Calibrations before and after the observation were obtained by scanning the narrow Ne $6599 \AA$ line under the same conditions as the observation, i.e. through the telescope.

For observations at ESO $3.6 \mathrm{~m}$ telescope the instrument is slightly the same but the aperture ratio is $\mathrm{F} / 2.5$ and the pixel size is $0.91^{\prime \prime}$ on the sky.

For CFHT observations we used SIS focal reducer in FP mode attached at F/8 Cassegrain focus. The CCD detector used was Loral 3 with $7.5 \mathrm{e}^{-}$of read out noise and pixel size on the sky is $0.68^{\prime \prime}$. Due to the read out noise it is impossible to have short exposures like IPCS, so typical time exposure is basically 5 min for each step and we scan only one cycle.

Different Perot-Fabry etalons were used, depending on the observed wavelength and on the velocity range in the galaxy (Table 2).

Different interference filters were also used to select redshifted emission lines, main characteristics are given in Table 3 .

\section{Data reduction}

The Perot-Fabry reducing software package ADHOC, developed at Marseille's Observatory (Boulesteix 1993) was used.

\subsection{IPCS observations}

In the first step individual channel images were added in order to build a data cube and observation cubes were the reduced into a wavelength calibrated cube $(x, y, \lambda)$. In the second step, the analyze of the detailed profile of the observed line within the free spectral range of the interferometer was done for each pixel. Then night sky and continuum subtractions are computed and velocity, monochromatic and continuum images were extracted.

\section{2. $C C D$ observations}

First step is to correct each image of the data cube from dark and flat field. It was done using images obtained observing the dome. After the flat field and dark corrections it is necessary to remove cosmic rays and correct the cube from transparency fluctuations and seeing variations. We had considerable changes in air mass during our exposure times often longer than 2 hours. This correction was made using field stars fluxes. To minimize seeing variations during one observation, we have smoothed each scan with a gaussian function with a FWHM equal to the worse seeing that we have in the data cube in order to have the same seeing in each channel. Then this clean cube is proceeded in the same manner as for IPCS observations. 
Table 2. Interferometers used for the observations

\begin{tabular}{ccccccc}
\hline Identification & $\begin{array}{c}\text { reference } \\
\text { wavelength }\end{array}$ & $\begin{array}{c}\text { interference } \\
\text { order }\end{array}$ & Finesse & $\begin{array}{c}\text { scanned } \\
\text { channels }\end{array}$ & $\begin{array}{c}\text { free spectral } \\
\text { range }\left(\mathrm{km} \mathrm{s}^{-1}\right)\end{array}$ & $\begin{array}{c}\text { Spectral } \\
\text { resolution }\end{array}$ \\
\hline$\# 1$ & $6563 \AA$ & 795 & 25 & 24 & 377.4 & 20000 \\
$\# 2$ & $6563 \AA$ & 501 & 21 & 32 & 598.8 & 10000 \\
\hline
\end{tabular}

Table 3. Journal of observations. SAO: $6 \mathrm{~m}$ telescope (Russia), ESO: $3.6 \mathrm{~m}$ telescope La Silla (Chile), CFHT: $3.6 \mathrm{~m}$ telescope Hawaii (USA)

\begin{tabular}{|c|c|c|c|c|c|c|c|c|}
\hline Name & $\begin{array}{l}\text { Coordinat } \\
\alpha \\
\end{array}$ & $\begin{array}{c}(\mathbf{1 9 5 0}) \\
\delta \\
\end{array}$ & $\begin{array}{c}\text { Date and place of } \\
\text { observations }\end{array}$ & $\begin{array}{c}\text { Time } \\
\text { Exposure (s) }\end{array}$ & $\begin{array}{c}\text { Interference filter } \\
\lambda \text { central,FWHM }\end{array}$ & $\begin{array}{l}\text { PF } \\
\text { Used }\end{array}$ & $\begin{array}{c}\text { Line } \\
\text { observed }\end{array}$ & $\begin{array}{c}\text { Pixel } \\
\text { size }\end{array}$ \\
\hline NGC 404 & $01^{\mathrm{h}} 06^{\mathrm{m}} 39.2^{\mathrm{s}}$ & $+35^{\mathrm{O}} 27^{\prime} 06^{\prime \prime}$ & $\begin{array}{l}\text { Oct.1993 (SAO) } \\
\text { Oct.1992 (SAO) } \\
\text { Aug.1991 (SAO) }\end{array}$ & $\begin{array}{l}8000 \\
9120 \\
7200\end{array}$ & $\begin{array}{l}6559 \AA, 13 \AA \\
6585 \AA, 14 \AA \\
5009 \AA, 58 \AA\end{array}$ & $\begin{array}{l}\# 2 \\
\# 1 \\
\# 1\end{array}$ & $\begin{array}{c}\mathrm{H}_{\alpha} \\
{[\mathrm{NII}] 6584 \AA} \\
{[\mathrm{OIII}] 5007 \AA}\end{array}$ & $\begin{array}{l}0.75^{\prime \prime} \\
0.67^{\prime \prime} \\
0.67^{\prime \prime}\end{array}$ \\
\hline NGC 708 & $01^{\mathrm{h}} 49^{\mathrm{m}} 50.0^{\mathrm{s}}$ & $+35^{\circ} 54^{\prime} 20^{\prime \prime}$ & $\begin{array}{l}\text { Oct.1994 (SAO) } \\
\text { Oct.1994 (SAO) }\end{array}$ & $\begin{array}{l}10240 \\
10880\end{array}$ & $\begin{array}{l}6668 \AA, 23 \AA \\
6687 \AA, 26 \AA\end{array}$ & $\begin{array}{l}\# 2 \\
\# 2\end{array}$ & $\begin{array}{c}\mathrm{H}_{\alpha} \\
{[\mathrm{NII}] 6584 \AA}\end{array}$ & $\begin{array}{l}0.92^{\prime \prime} \\
0.92^{\prime \prime}\end{array}$ \\
\hline NGC 1052 & $02^{\mathrm{h}} 38^{\mathrm{m}} 37.4^{\mathrm{s}}$ & $-08^{\mathrm{O}} 28^{\prime} 10^{\prime \prime}$ & $\begin{array}{l}\text { Oct.1992 (SAO) } \\
\text { Oct.1994 (SAO) } \\
\text { Oct.1993 (SAO) }\end{array}$ & $\begin{array}{l}17760 \\
13200 \\
13240\end{array}$ & $\begin{array}{l}6617 \AA, 11 \AA \\
6617 \AA, 11 \AA \\
6602 \AA, 11 \AA\end{array}$ & $\begin{array}{l}\# 1 \\
\# 2 \\
\# 2\end{array}$ & $\begin{array}{c}{[\mathrm{NII}] 6584 \AA} \\
{[\mathrm{NII}] 6584 \AA} \\
\mathrm{H}_{\alpha}\end{array}$ & $\begin{array}{l}0.67^{\prime \prime} \\
0.92^{\prime \prime} \\
0.75^{\prime \prime}\end{array}$ \\
\hline NGC 2974 & $09^{\mathrm{h}} 40^{\mathrm{m}} 01.9^{\mathrm{s}}$ & $-03^{\circ} 28^{\prime} 15^{\prime \prime}$ & $\begin{array}{l}\text { Fev.1995 (CFHT) } \\
\text { Fev.1995 (CFHT) }\end{array}$ & $\begin{array}{l}9600 \\
9600\end{array}$ & $\begin{array}{l}6628 \AA, 12 \AA \\
6606 \AA, 25 \AA\end{array}$ & $\begin{array}{l}\# 2 \\
\# 2\end{array}$ & $\begin{array}{c}{[\mathrm{NII}] 6584 \AA} \\
\mathrm{H}_{\alpha}\end{array}$ & $\begin{array}{l}0.68^{\prime \prime} \\
0.68^{\prime \prime}\end{array}$ \\
\hline NGC 5846 & $15^{\mathrm{h}} 03^{\mathrm{m}} 57.0^{\mathrm{s}}$ & $+01^{\circ} 47^{\prime} 57^{\prime \prime}$ & May.1990 (ESO) & 12000 & $6608 \AA ⿻, 31 \AA$ & $\# 1$ & [NII] $6584 \AA$ & $0.91^{\prime \prime}$ \\
\hline NGC 5866 & $15^{\mathrm{h}} 05^{\mathrm{m}} 07.0^{\mathrm{s}}$ & $+55^{\circ} 57^{\prime} 16^{\prime \prime}$ & $\begin{array}{l}\text { Oct.1992 (SAO) } \\
\text { Oct.1992 (SAO) }\end{array}$ & $\begin{array}{l}5760 \\
7200\end{array}$ & $\begin{array}{l}6586 \AA, 14 \AA \\
6603 \AA, 13 \AA\end{array}$ & $\begin{array}{l}\# 1 \\
\# 1\end{array}$ & $\begin{array}{c}\mathrm{H}_{\alpha} \\
{[\mathrm{NII}] 6584 \AA}\end{array}$ & $\begin{array}{l}0.67^{\prime \prime} \\
0.67^{\prime \prime}\end{array}$ \\
\hline NGC 5898 & $15^{\mathrm{h}} 15^{\mathrm{m}} 17.0^{\mathrm{s}}$ & $-23^{\circ} 54^{\prime} 54^{\prime \prime}$ & Fev.1995 (CFHT) & 7040 & $6611 \AA ⿻, 10 \AA$ & $\# 2$ & $\mathrm{H}_{\alpha}$ & $0.68^{\prime \prime}$ \\
\hline NGC 6868 & $20^{\mathrm{h}} 06^{\mathrm{m}} 16.0^{\mathrm{s}}$ & $-48^{\circ} 31^{\prime} 36^{\prime \prime}$ & Aug.1995 (ESO) & 9600 & $6646 \AA, 24 \AA$ & $\# 2$ & [NII] $6584 \AA$ & $0.91^{\prime \prime}$ \\
\hline NGC 7014 & $21^{\mathrm{h}} 04^{\mathrm{m}} 29.0^{\mathrm{s}}$ & $-47^{\mathrm{O}} 22^{\prime} 48^{\prime \prime}$ & Aug.1995 (ESO) & 11400 & $6694 \AA, 27 \AA$ & $\# 2$ & [NII] $6584 \AA$ & $0.91^{\prime \prime}$ \\
\hline NGC 7332 & $22^{\mathrm{h}} 35^{\mathrm{m}} 00.1^{\mathrm{s}}$ & $+23^{\mathrm{O}} 32^{\prime} 16^{\prime \prime}$ & $\begin{array}{l}\text { Oct.1993 (SAO) } \\
\text { Oct.1993 (SAO) } \\
\text { Oct.1994 (SAO) }\end{array}$ & $\begin{array}{c}8320 \\
8320 \\
12160\end{array}$ & $\begin{array}{l}6602 \AA, 11 \AA \\
6587 \AA, 14 \AA \\
6587 \AA, 14 \AA\end{array}$ & $\begin{array}{l}\# 2 \\
\# 2 \\
\# 2\end{array}$ & $\begin{array}{c}{[\mathrm{NII}] 6584 \AA} \\
\mathrm{H}_{\alpha} \\
\mathrm{H}_{\alpha}\end{array}$ & $\begin{array}{l}0.75^{\prime \prime} \\
0.75^{\prime \prime} \\
0.92^{\prime \prime}\end{array}$ \\
\hline
\end{tabular}

\subsection{Phase correction}

The phase map, obtained from the calibration cubes, is used to calibrate in wavelength observation cubes. The accuracy of the zero wavelength is a fraction of a channel width $\left(<3 \mathrm{~km} \mathrm{~s}^{-1}\right)$ over the whole field.

\subsection{Night sky subtraction}

In the red spectra $\left(\mathrm{H}_{\alpha},[\mathrm{NII}] 6584 \AA\right)$, it is necessary to subtract the night sky $\mathrm{OH}$ lines passing through the narrow filter which are often of the same intensity as the gas emission lines we observe. This is done by determining the $\mathrm{OH}$ profile from areas of the field sufficiently far from the center of the galaxy to be supposed free from any galactic emission (Laval et al. 1987).

\subsection{Continuum modulation}

Even if the width of the filter is large compared to the free spectral range a modulation can appear on observed spectra, due mainly to the strong continuum of elliptical galaxies. Observation of a continuum lamp was used to correct from this effect.

\subsection{Velocities, monochromatic and continuum images}

From the individual spectra calibrated in wavelength the continuum map was easily derived and represents for each pixel the value of the mean of the three faintest channels, in order to avoid channel noise effect. To obtain monochromatic images is more delicate because, in the case of multiple components profiles, different maps can be produced for each component. In this case, it is first necessary to decompose multiple profiles in order to build cubes corresponding to each component separately. A gaussian algorithm fitting is used to decompose complex profiles. 

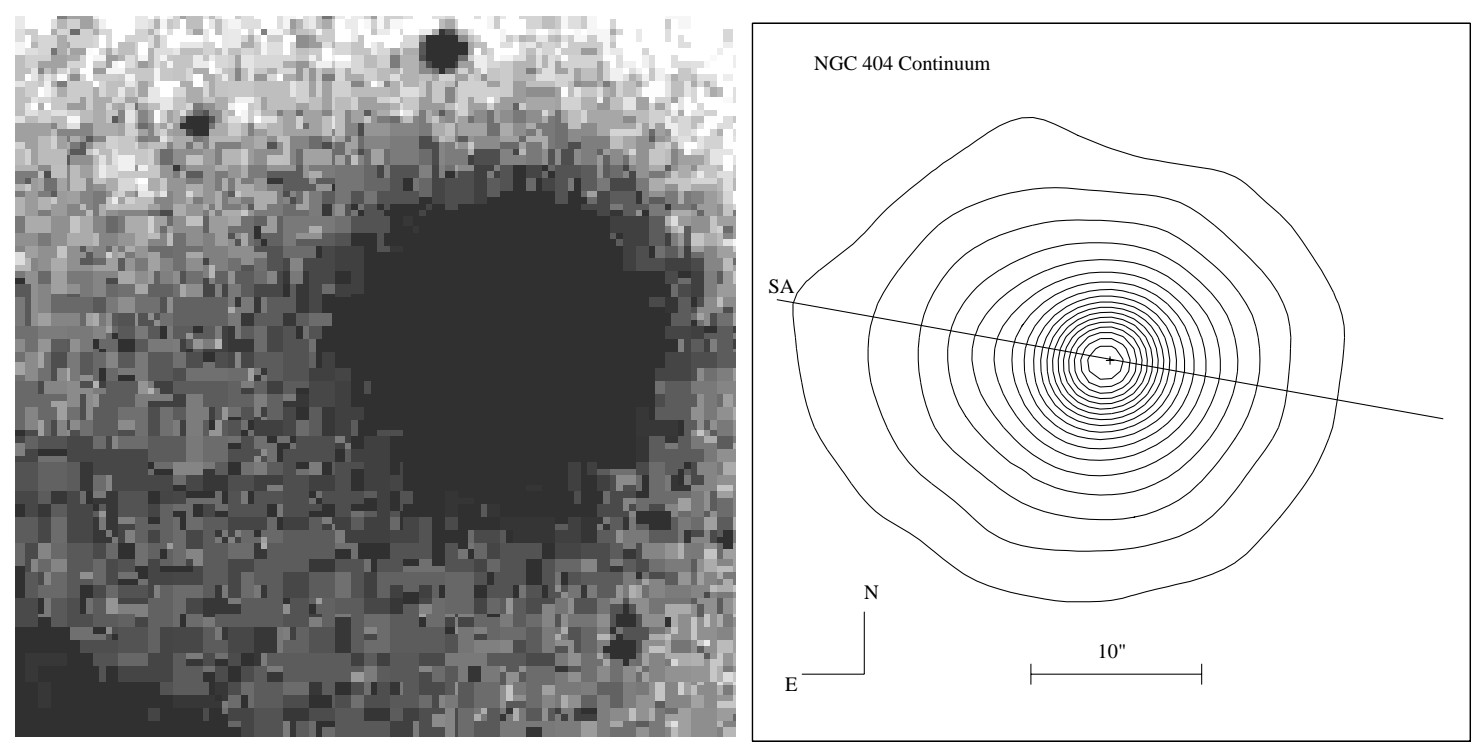

Fig. 1. NGC 404: First is red image from Digitalized Sky Survey with North on the top and East on the left. Image total size is 3 arcmin. Second is continuum image of NGC 404 from our observations, SA represents the stellar axis

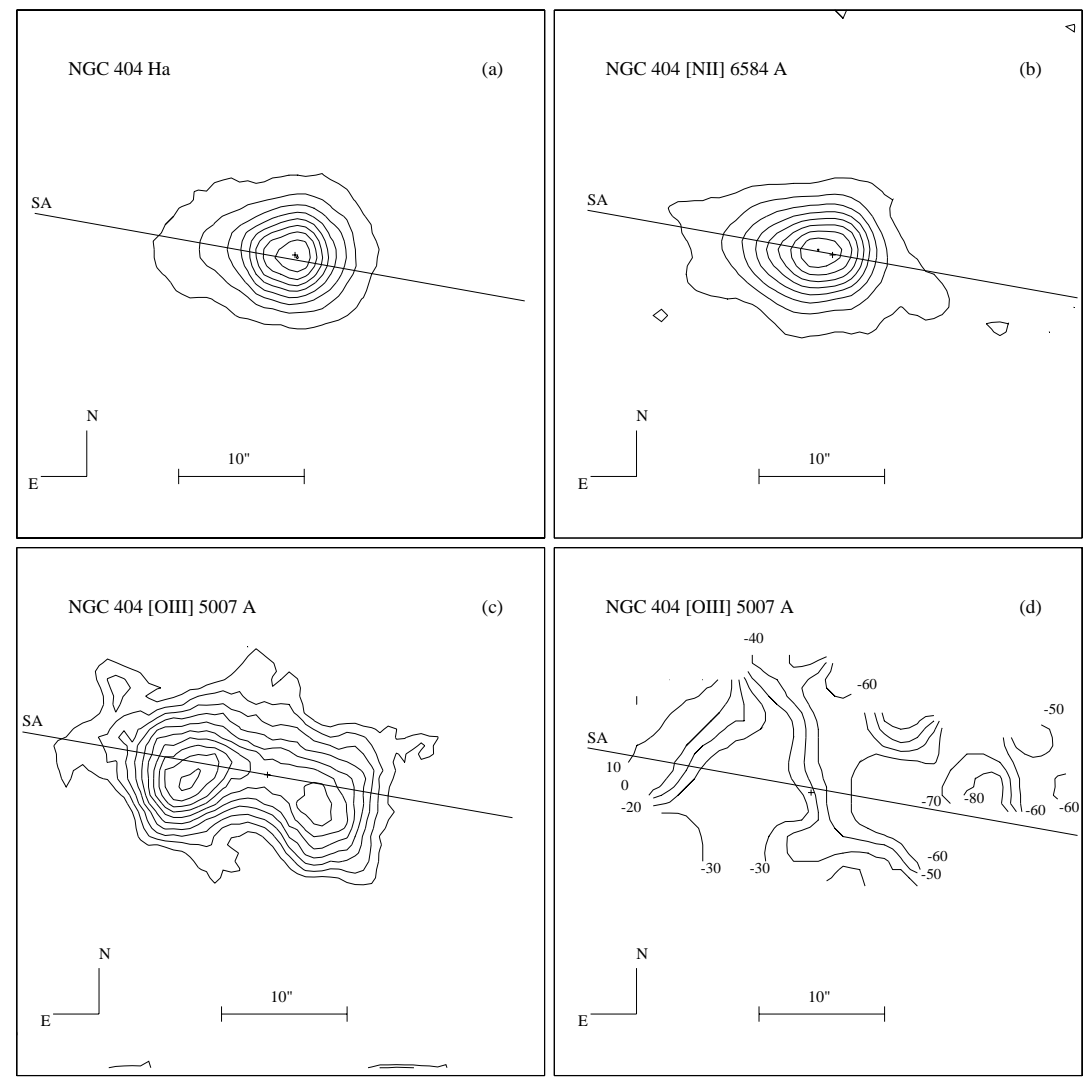

Fig. 2. NGC 404: a) $\mathrm{H}_{\alpha}$ monochromatic map, contours are in units of $10^{-17} \mathrm{erg} \mathrm{s}^{-1} \mathrm{~cm}^{-2} \operatorname{arcsec}^{-2}$. Lowest is 6.6 and step 16.6 . b) $[\mathrm{NII}] 6584 \AA$ map, contours are in same units with lowest level of 7.5 and step 6.6. c) [OIII] $5007 \AA$ map (not calibrated). d) Velocity map derived from [OIII] $5007 \AA$ observation. SA represents the stellar axis 

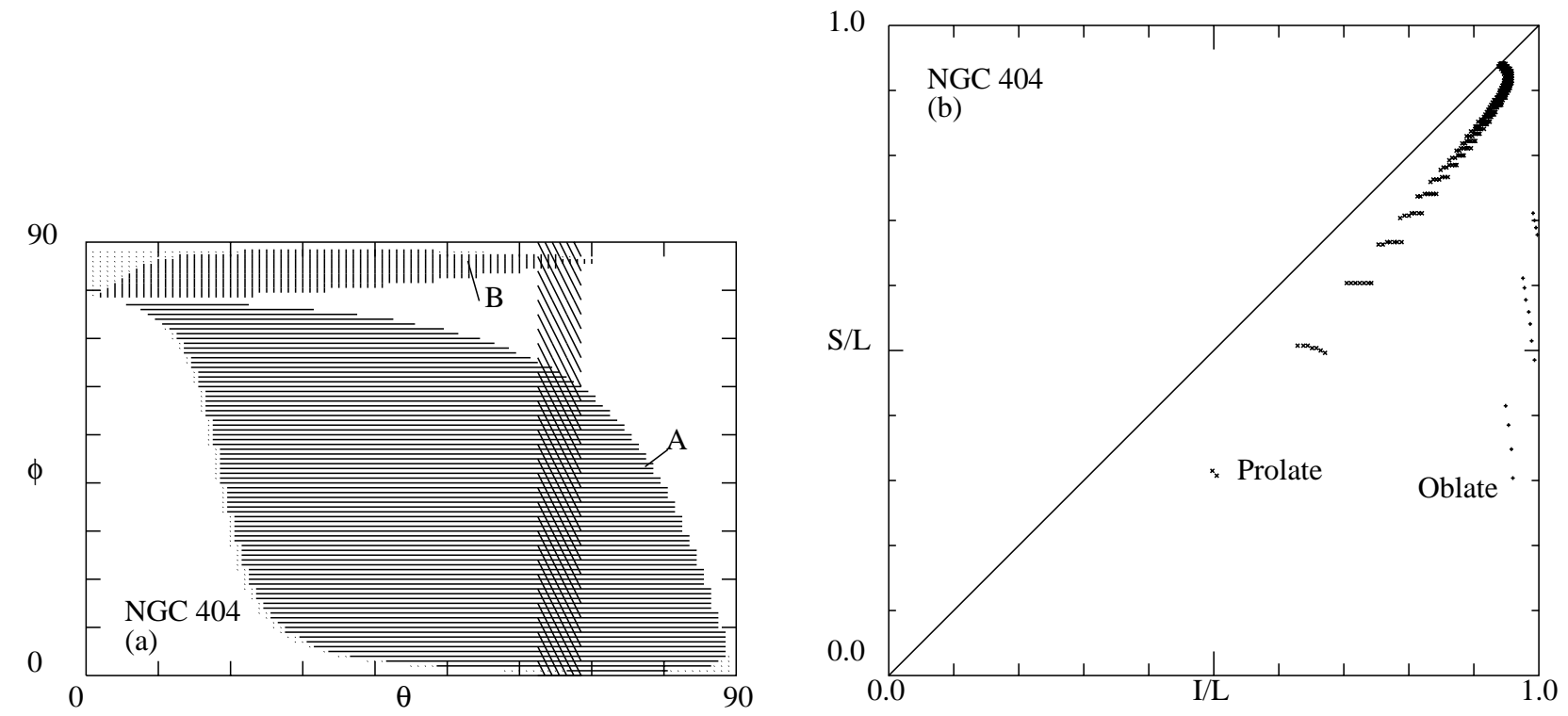

Fig. 3. NGC 404: a) Possible viewing angles in the $\theta-\phi$ plane. Case A: gas $\perp$ short axis. Case B gas $\perp$ long axis. b) Possible axis ratios for the ellipsoid ( $S=$ short, $I=$ intermediate, $L=$ long) in accordance of $\theta$ values of showed in a)
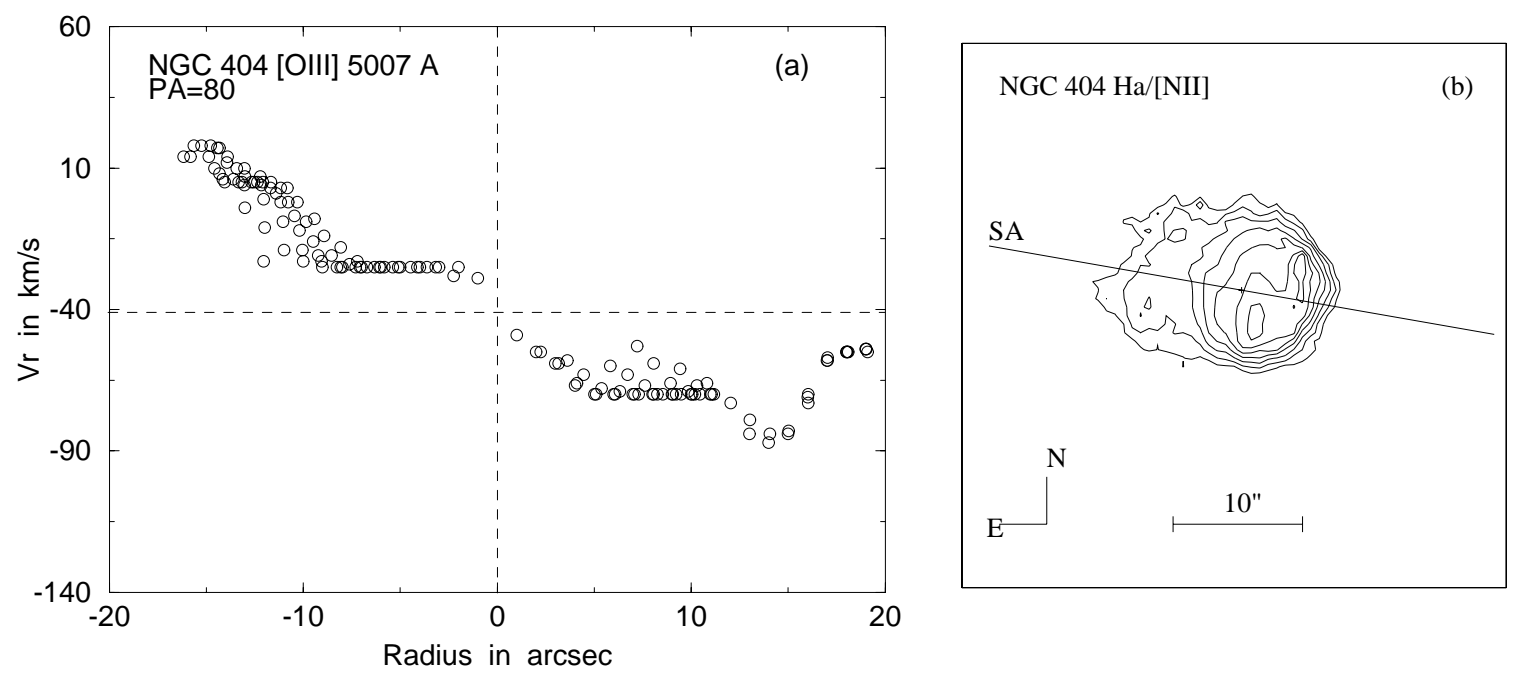

Fig. 4. NGC 404: a) Line of sight velocity diagram for the gas with respect of a PA $=80^{\circ}$. b) $\mathrm{H}_{\alpha} /[\mathrm{NII}]$ map, relative lowest level is 80 and step is 30 

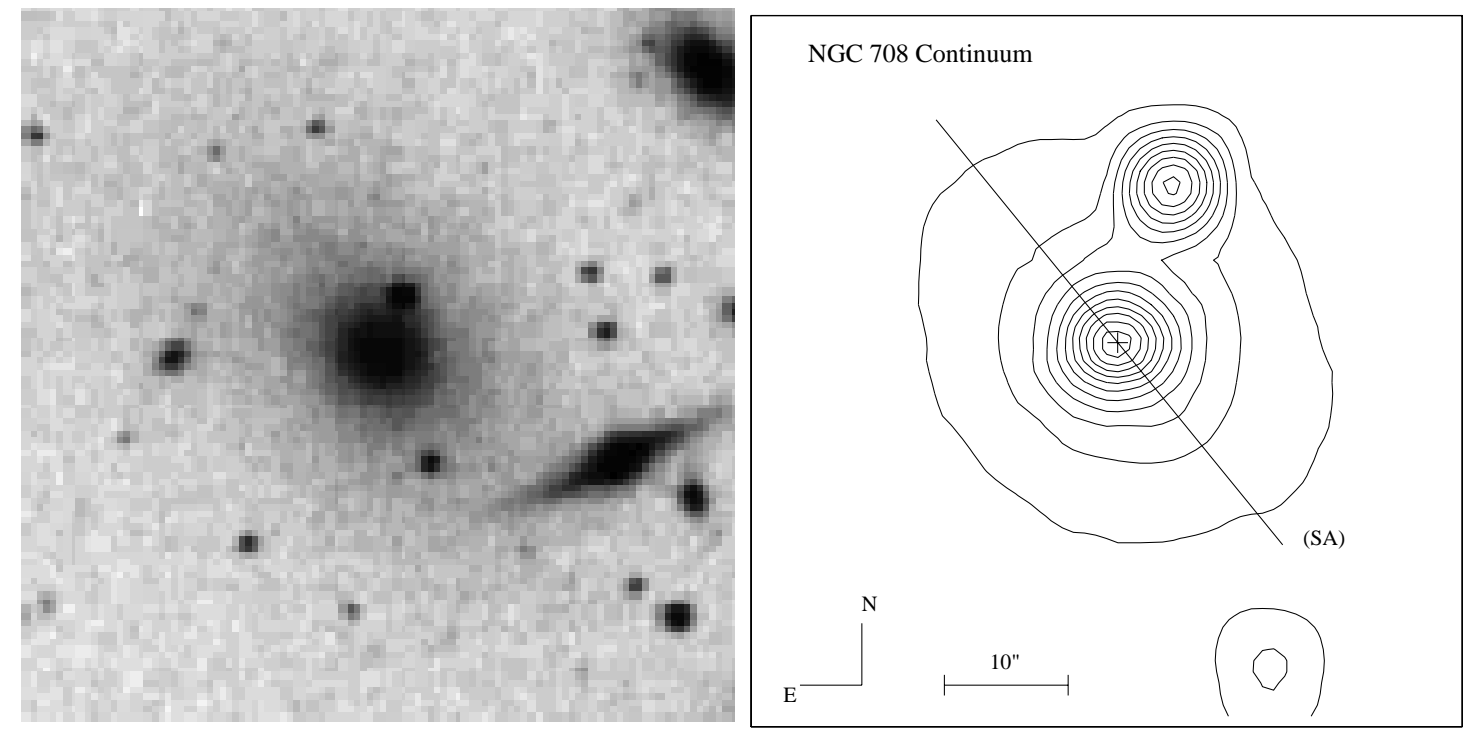

Fig. 5. NGC 708: Same as NGC 404

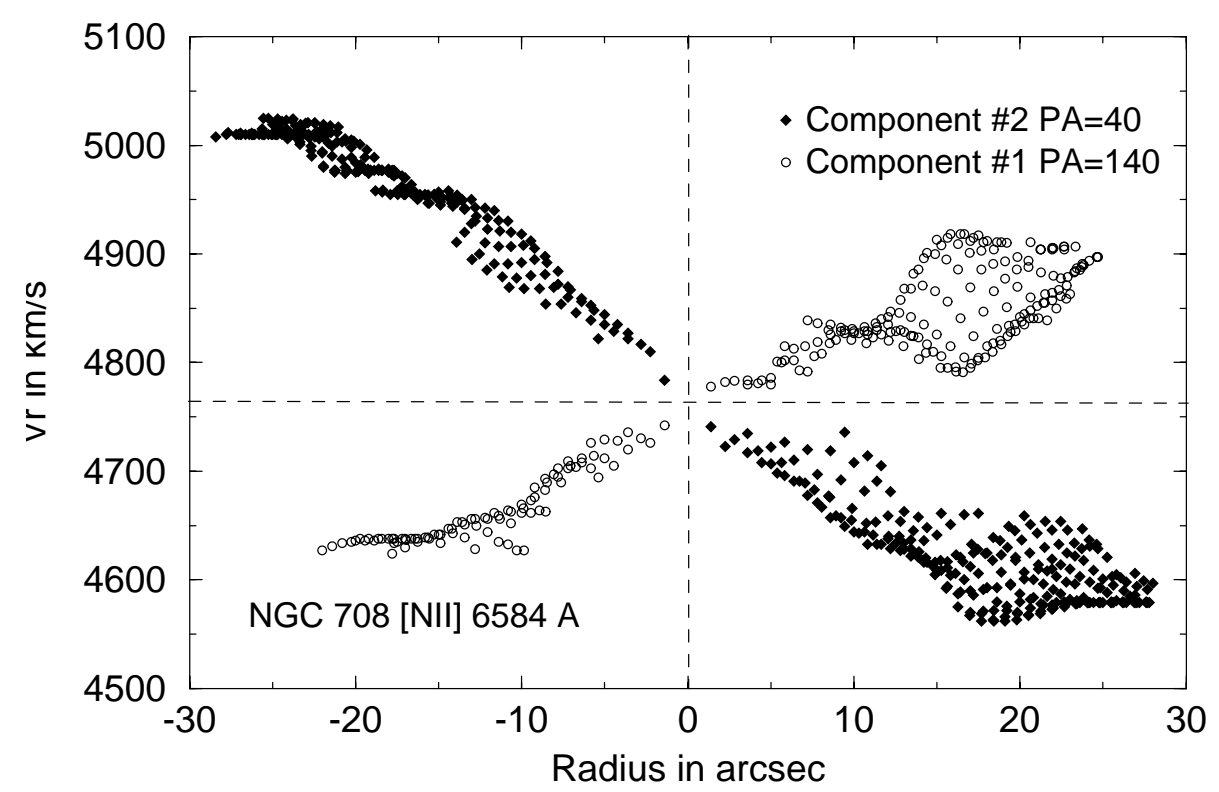

Fig. 6. NGC 708: Line of sight velocity diagram of the two gaseous components 

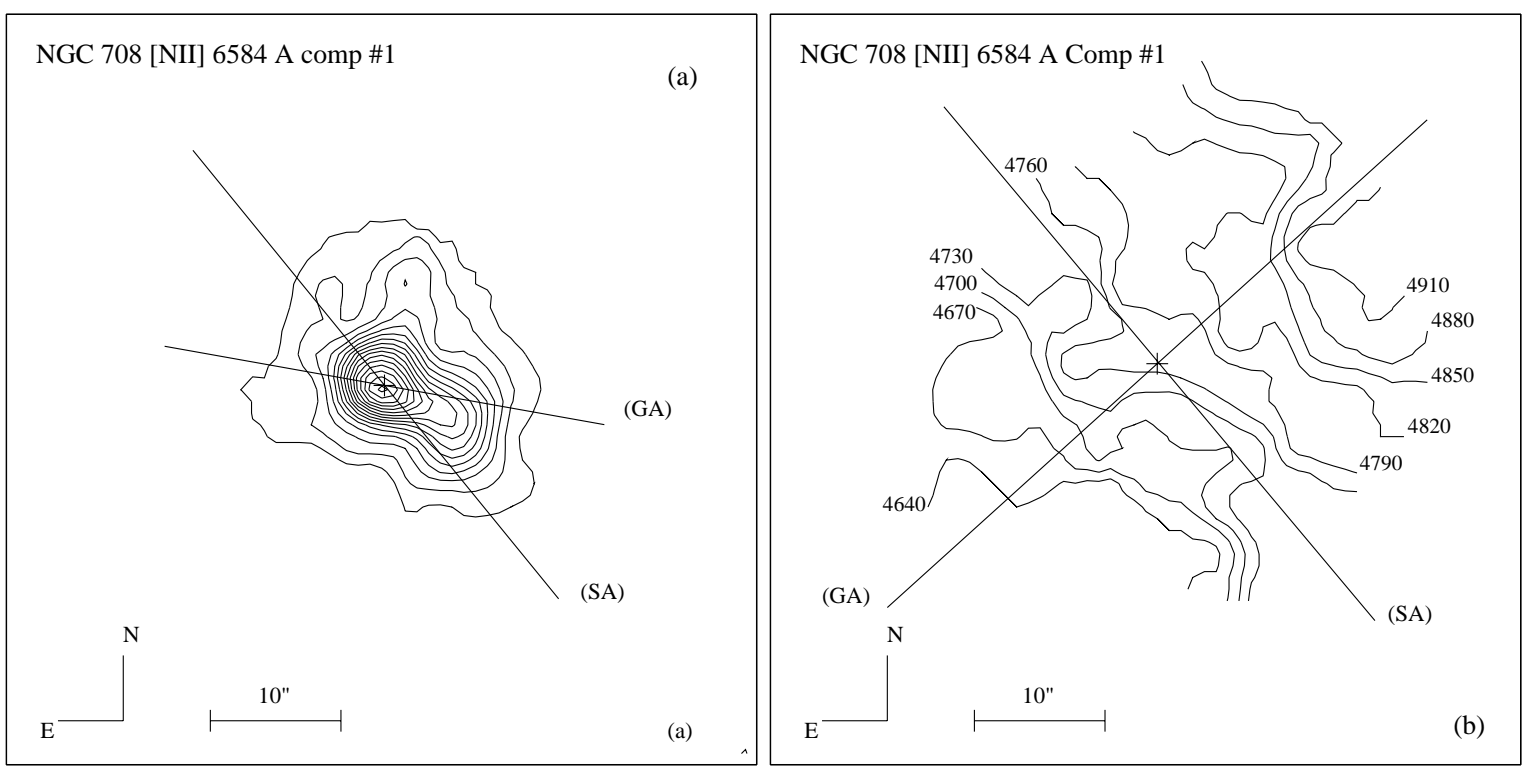

Fig. 7. NGC 708 Component \#1: a) [NII] $6584 \AA$ Monochromatic map. Contours are in units of $10^{-17} \mathrm{erg} \mathrm{s}^{-1} \mathrm{~cm}^{-2} \operatorname{arcsec}^{-2}$. Lowest 4 and step 2.7. b) Velocity map. SA is the stellar axis and GA the gas axis (which is different for velocity and monochromatic maps)
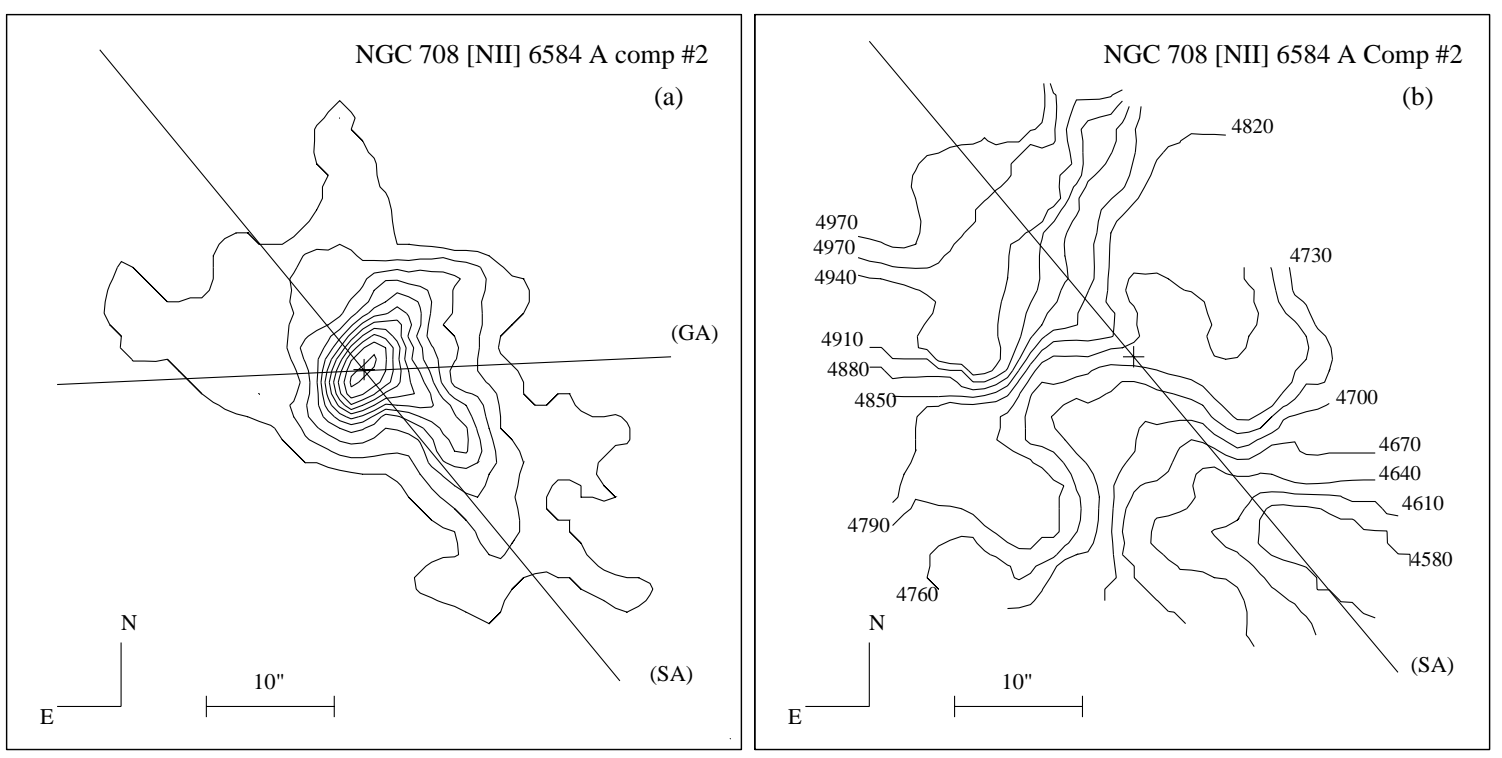

Fig. 8. NGC 708 Component \#2: a) [NII] $6584 \AA$ Monochromatic map. Contours are in units of $10^{-17} \mathrm{erg} \mathrm{s}^{-1} \mathrm{~cm}^{-2} \mathrm{arcsec}^{-2}$. Lowest 1.5 and step $1.35 \mathrm{~b}$ ) Velocity map. SA is the stellar axis and GA the gas axis (which is different for velocity and monochromatic maps) 

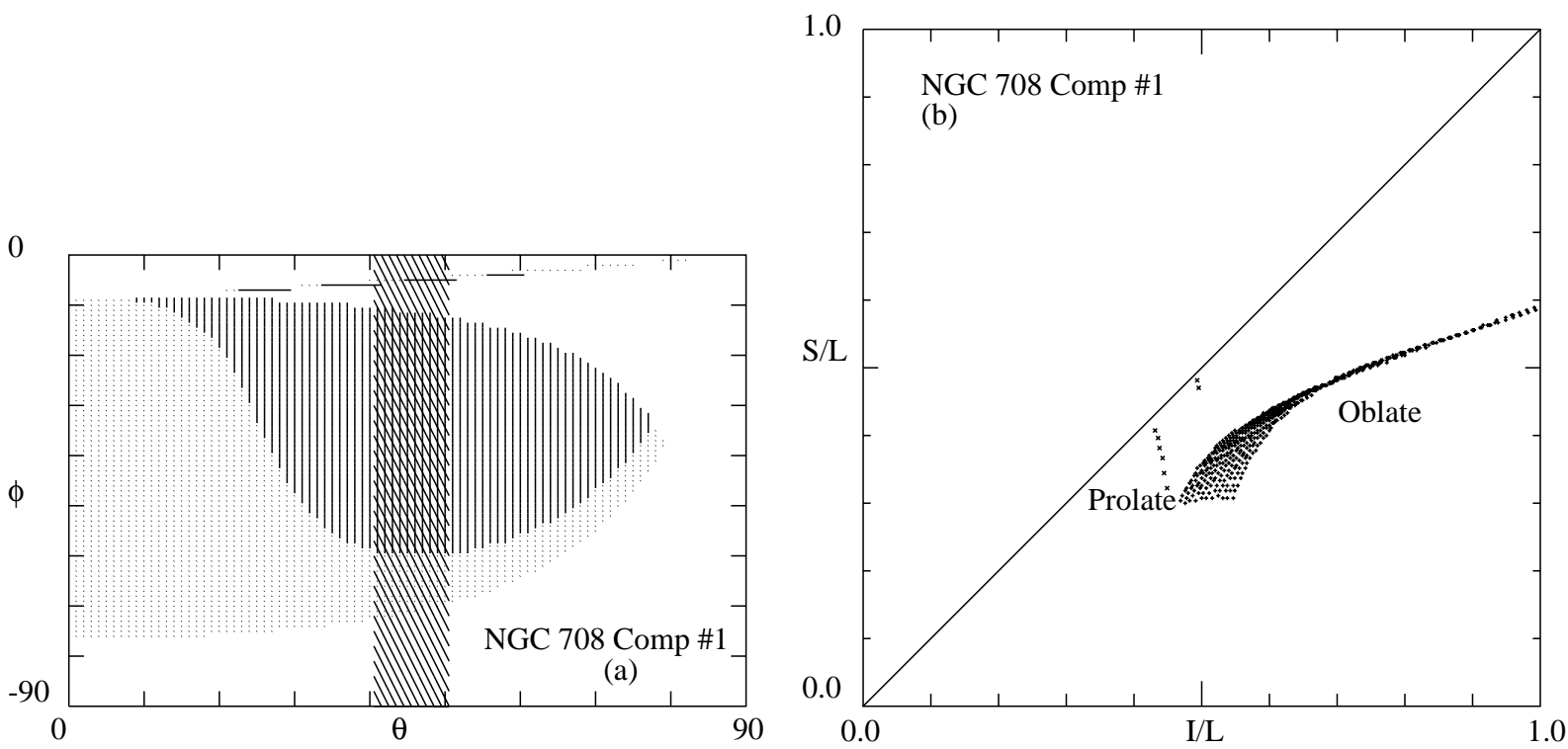

Fig. 9. NGC 708 Component \#1: Possible viewing angles a) and axis ratios b)
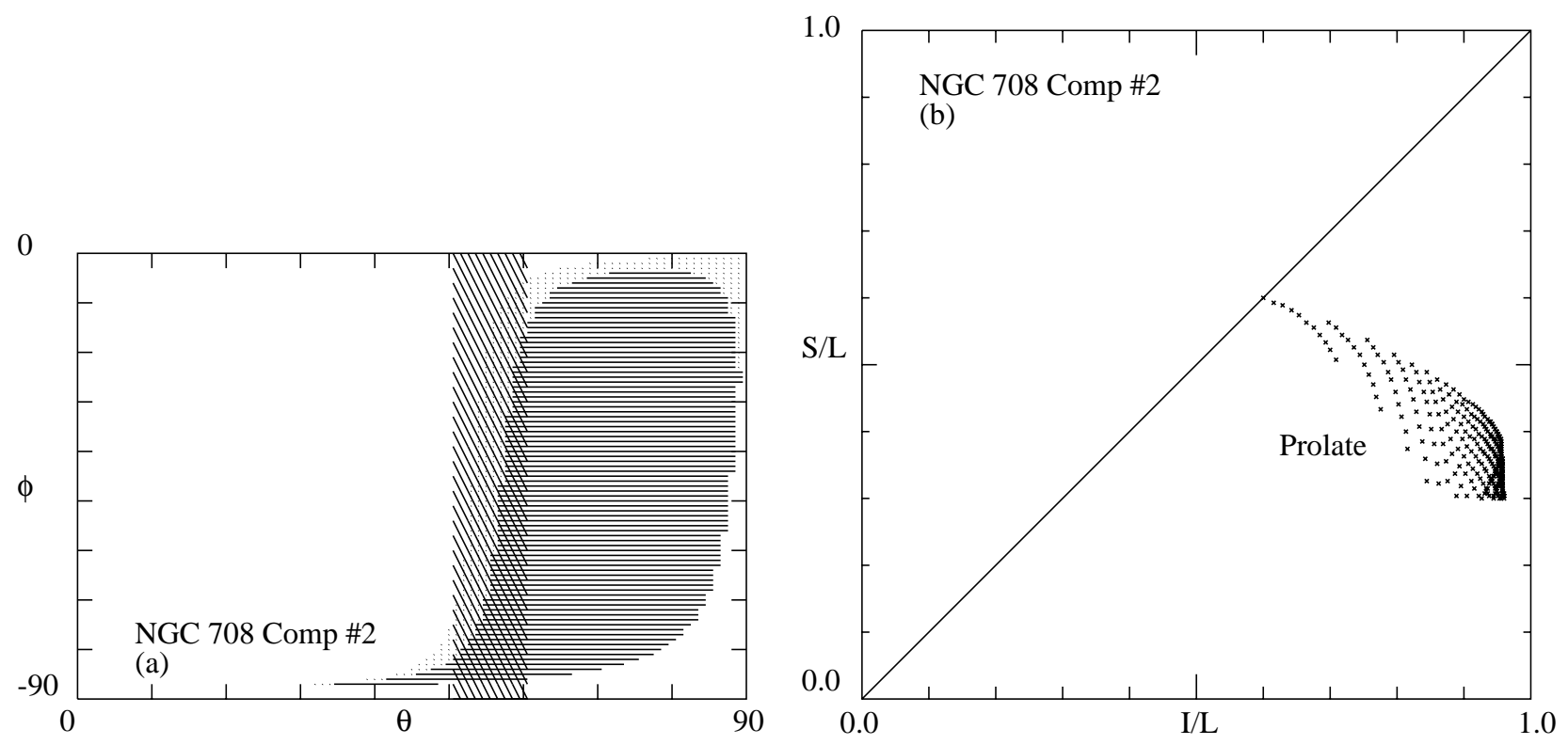

Fig. 10. NGC 708 Component \#2: Possible viewing angles a) and axis ratios b) 

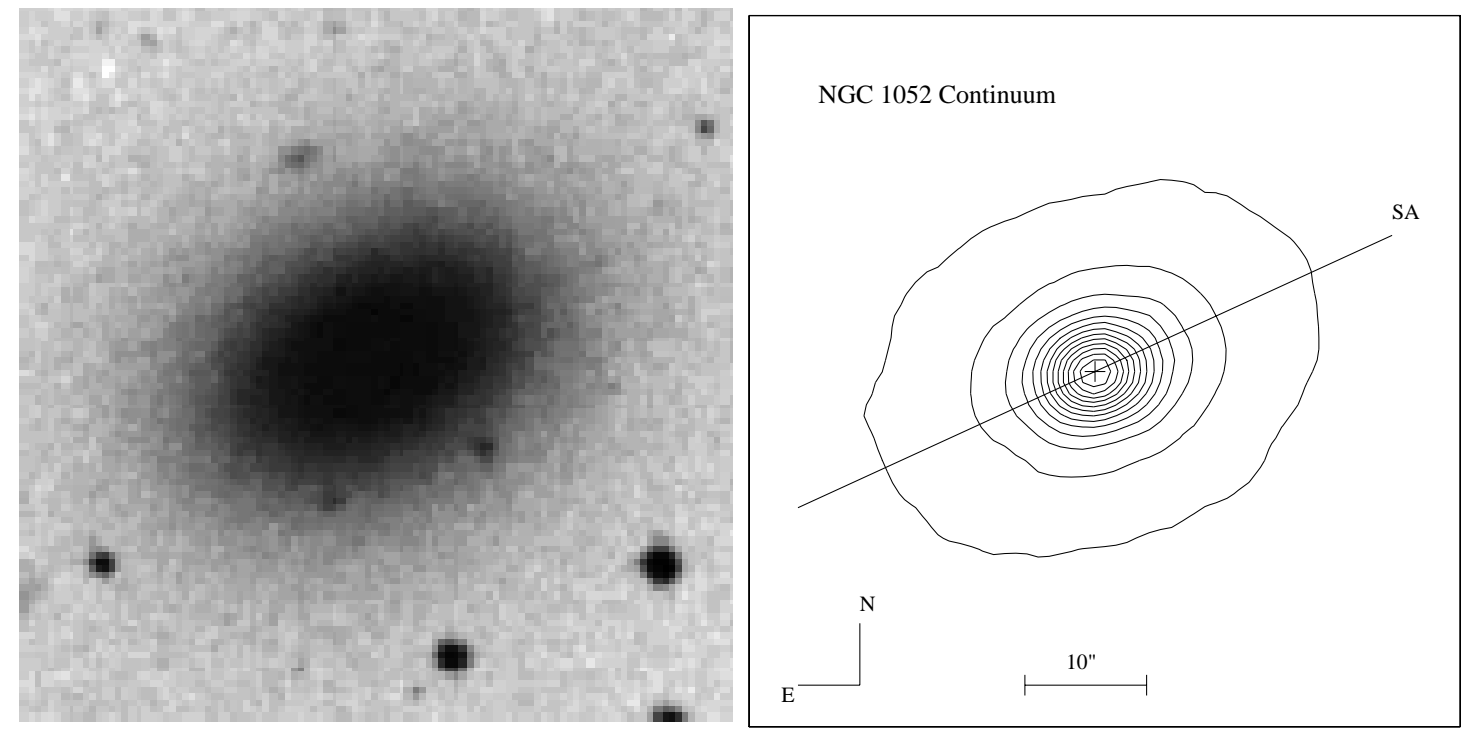

Fig. 11. NGC 1052: Same as NGC 404

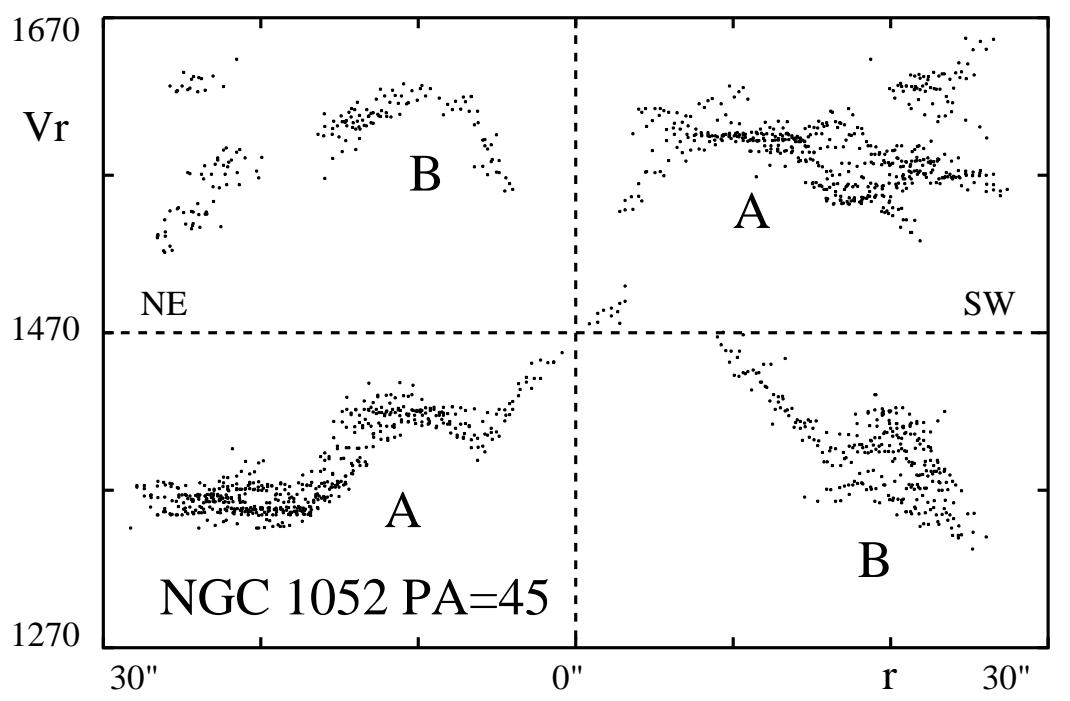

Fig. 12. NGC 1052: Line of sight velocity diagram of the two gaseous components. A: Major component. B: Second component 

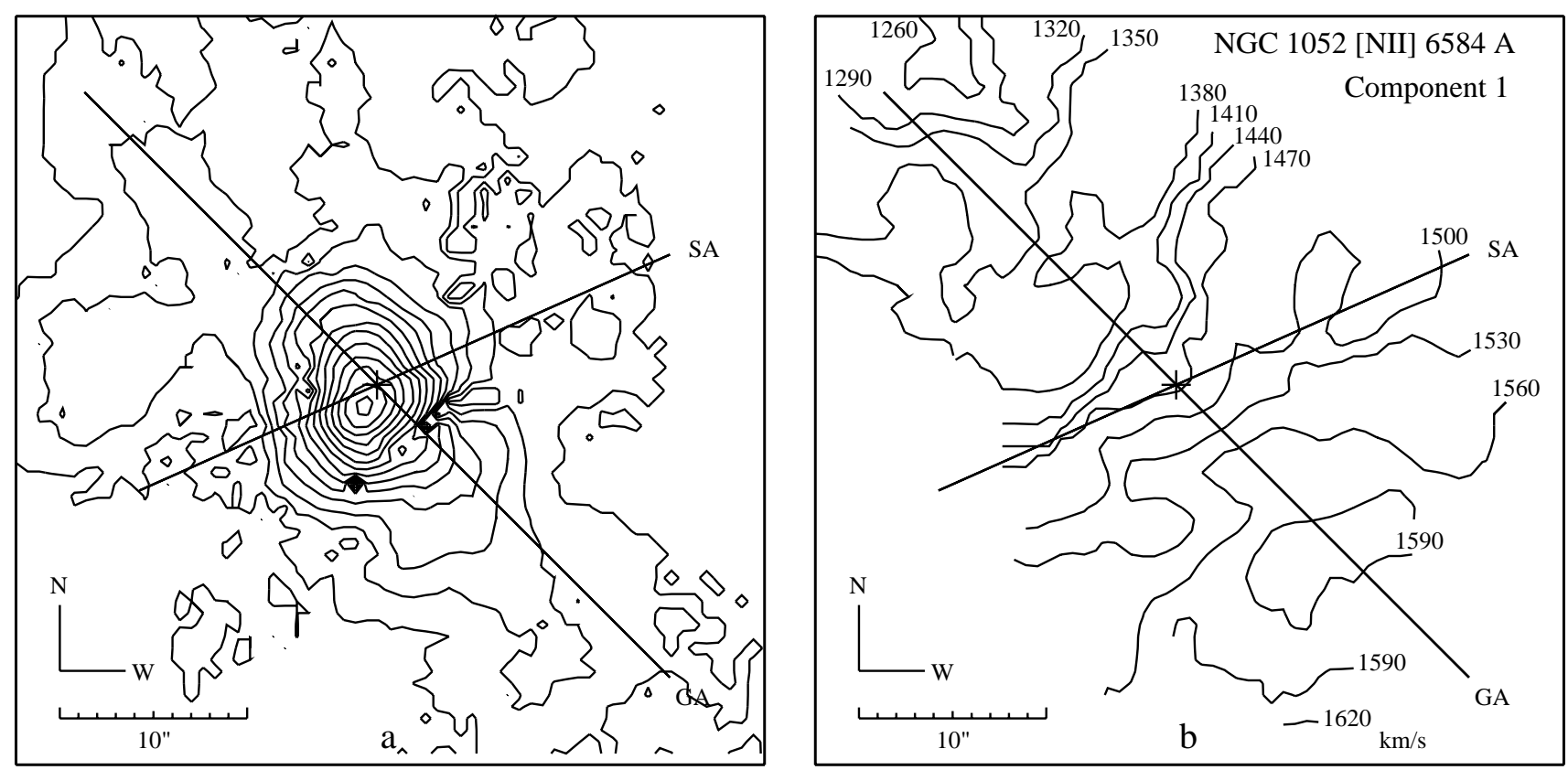

Fig. 13. NGC 1052 Component \#1: a) [NII] $6584 \AA$ Monochromatic map. Contours are in units of $10^{-17} \mathrm{erg} \mathrm{s}^{-1} \mathrm{~cm}^{-2} \mathrm{arcsec}^{-2}$. Lowest 3 and step 7. b) Velocity map. SA is the stellar axis and GA the gas axis (which is different for velocity and monochromatic maps)
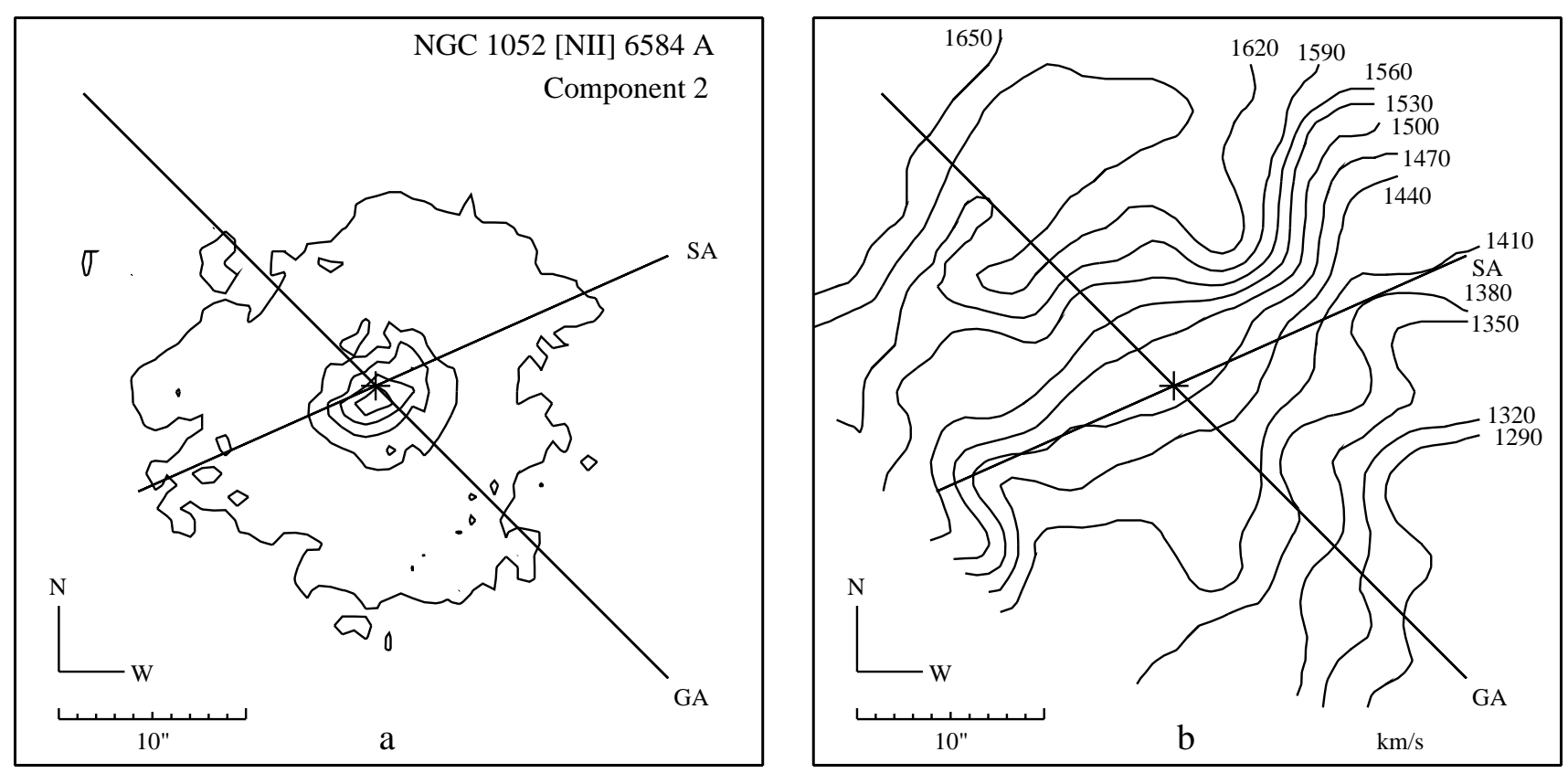

Fig. 14. NGC 1052 Component \#2: a) [NII] $6584 \AA$ Monochromatic map. Contours are in units of $10^{-17} \mathrm{erg} \mathrm{s}^{-1} \mathrm{~cm}^{-2} \mathrm{arcsec}^{-2}$. Lowest 3 and step 7. b) Velocity map. SA is the stellar axis and GA the gas axis (which is different for velocity and monochromatic maps) 


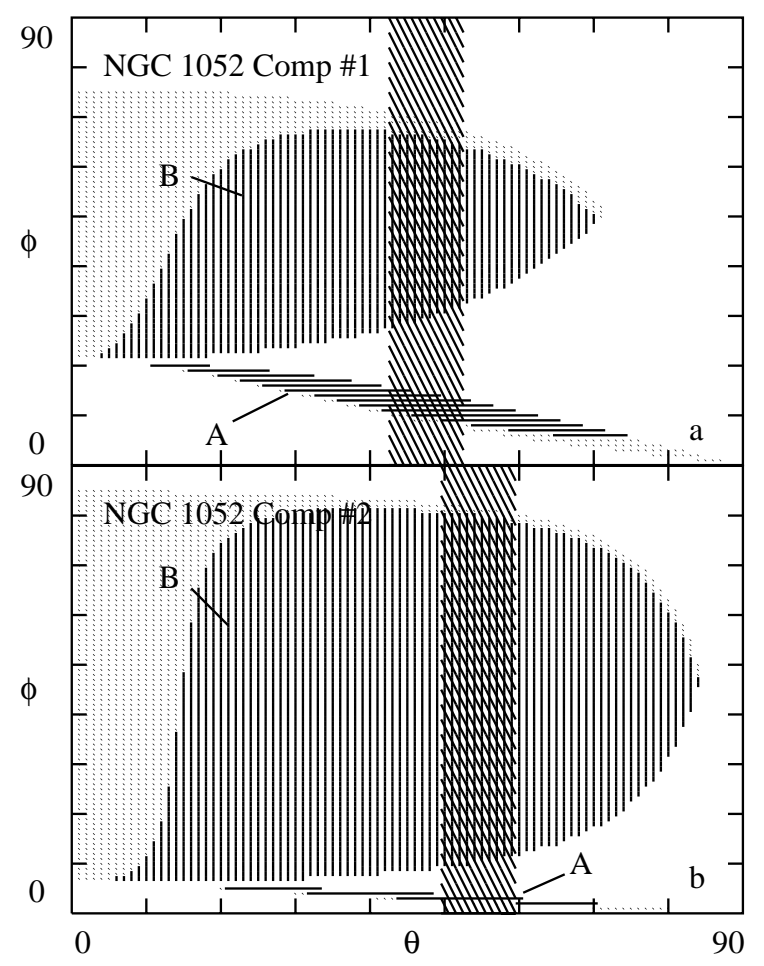

Fig. 15. NGC 1052: Possible viewing angles for component \#1 a) and component \#2 b). Case A: gas $\perp$ short axis. Case B gas $\perp$ long axis
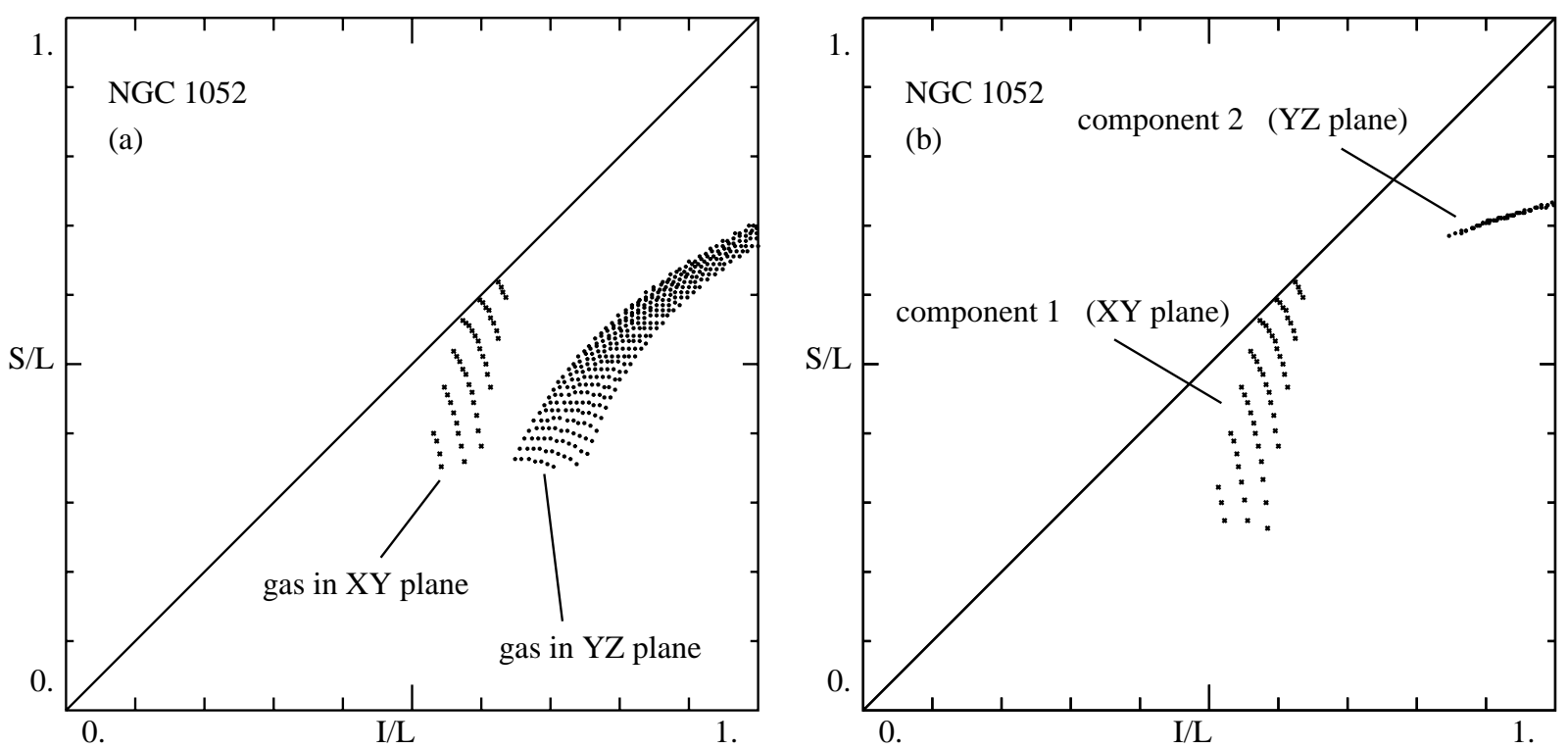

Fig. 16. NGC 1052: Possible axis ratios for component \#1 a) and for the hypothesis of $5^{\circ}<\phi<15^{\circ}$ b) 

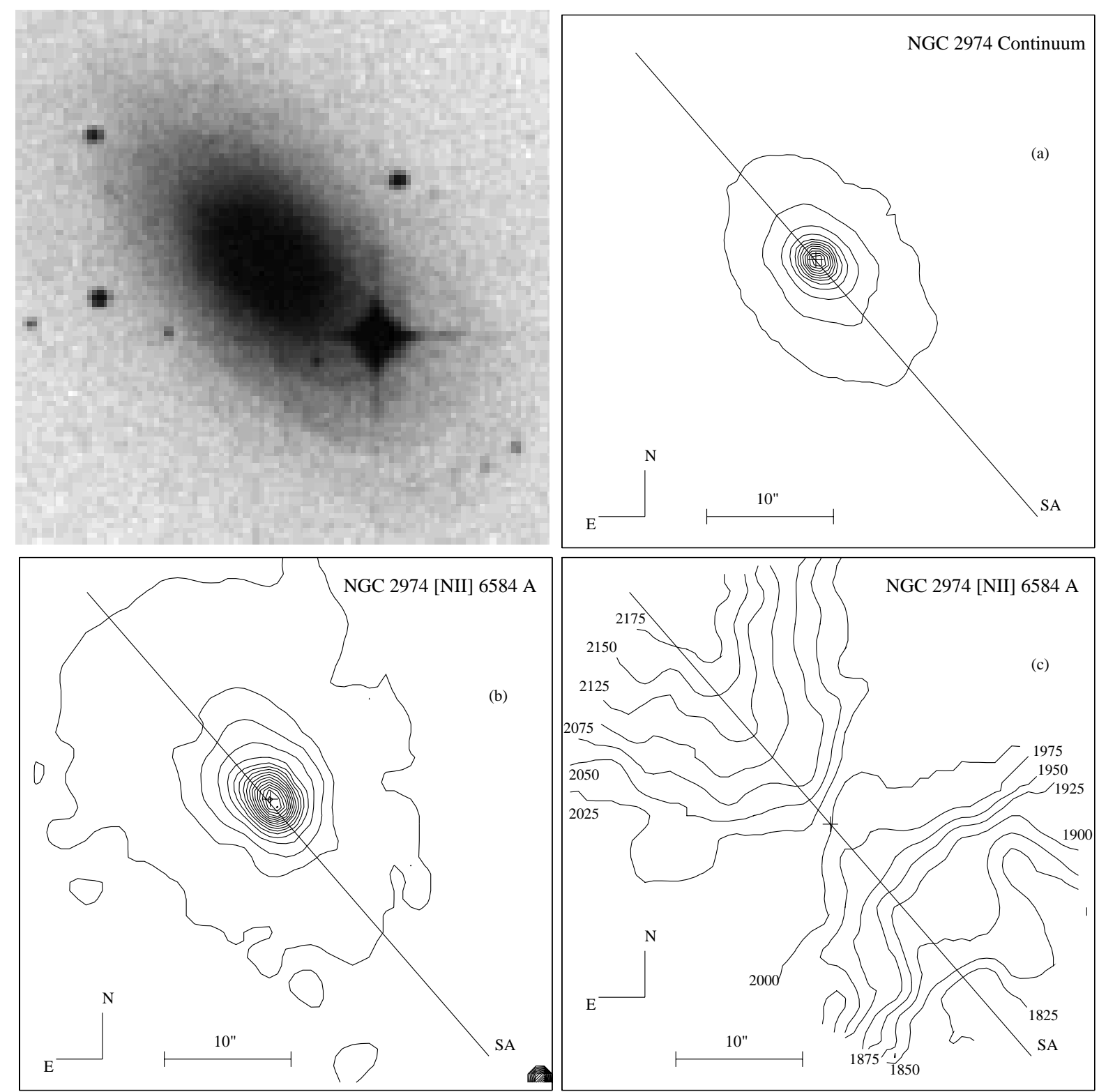

Fig. 17. NGC 2974: Field image from Digitalized Sky Survey, total size is 3 arcmin. a) Continuum image from our observations. b) $[\mathrm{NII}] 6584 \AA$ Å monochromatic map. Contours are in units of $10^{-17} \mathrm{erg} \mathrm{s}^{-1} \mathrm{~cm}^{-2} \operatorname{arcsec}^{-2}$. Lowest 4.1 and step 22.5. c) Velocity field. SA represents the stellar axis 


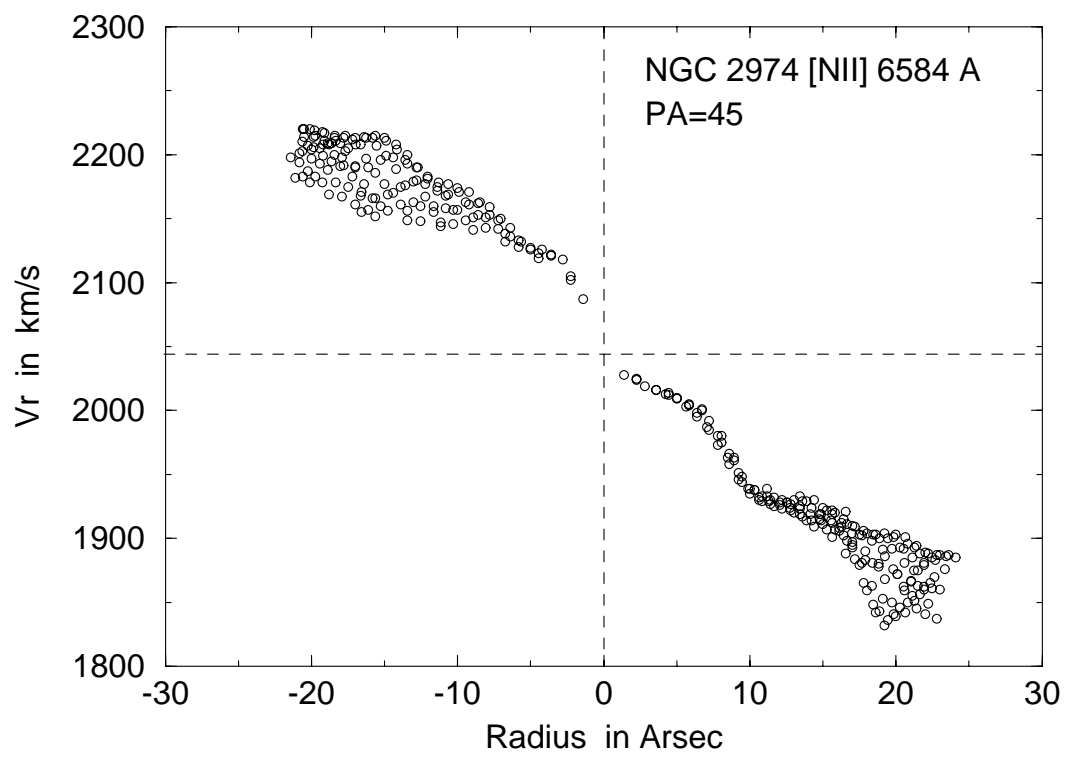

Fig. 18. NGC 2974: Line of sight velocity diagram
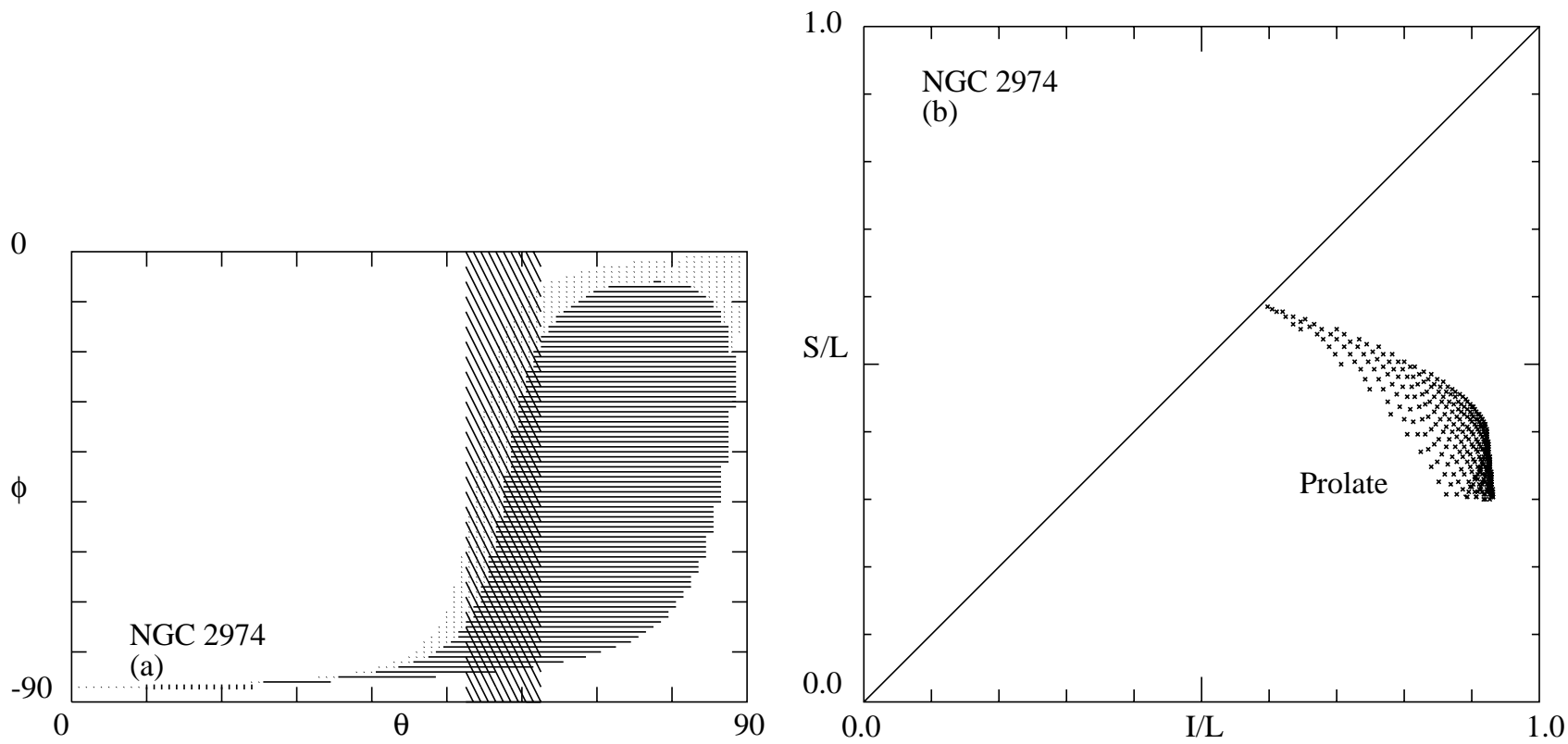

Fig. 19. NGC 2974: a) Possible viewing angles in the $\theta-\phi$ plane. b) Possible axis ratios for the ellipsoid $(S=$ short, $I=$ intermediate, $L=$ long) in accordance of $\theta$ values of showed in a) 

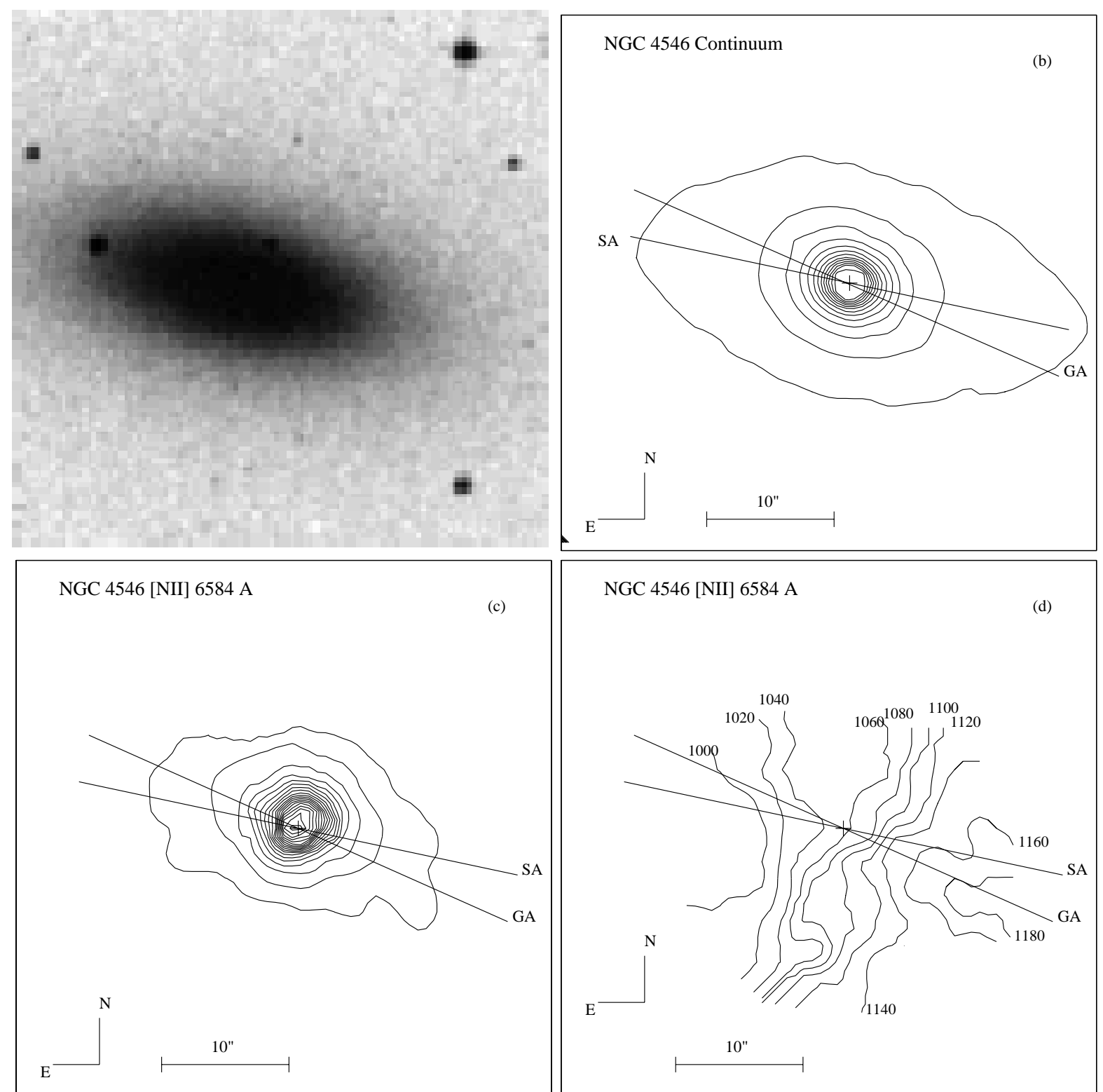

NGC 4546 [NII] 6584 A

(d)

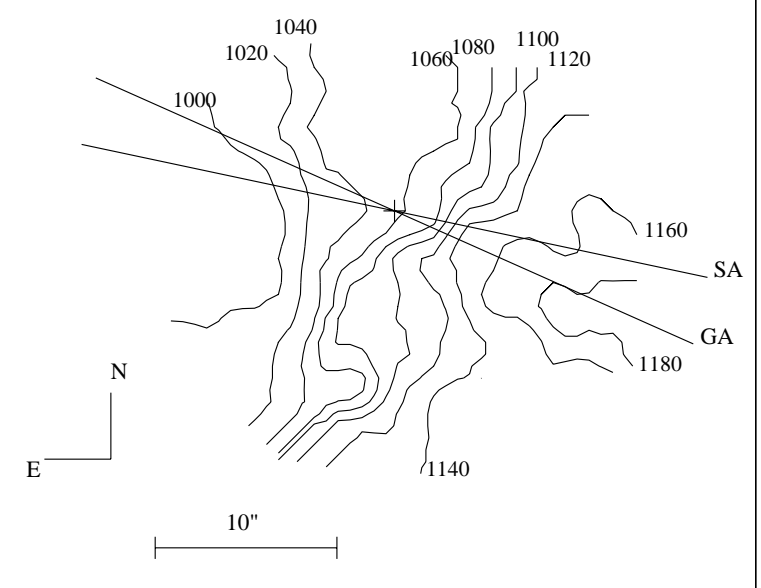

Fig. 20. NGC 4546: Field image from Digitalized Sky Survey, total size is 3 arcmin. a) Continuum image from our observations. b) $[\mathrm{NII}] 6584 \AA$ monochromatic map. Contours are in units of $10^{-17} \mathrm{ergs}^{-1} \mathrm{~cm}^{-2} \operatorname{arcsec}^{-2}$. Lowest 15.6 and step 16.4. c) Velocity field. SA represents the stellar axis, GA represents the gas axis 


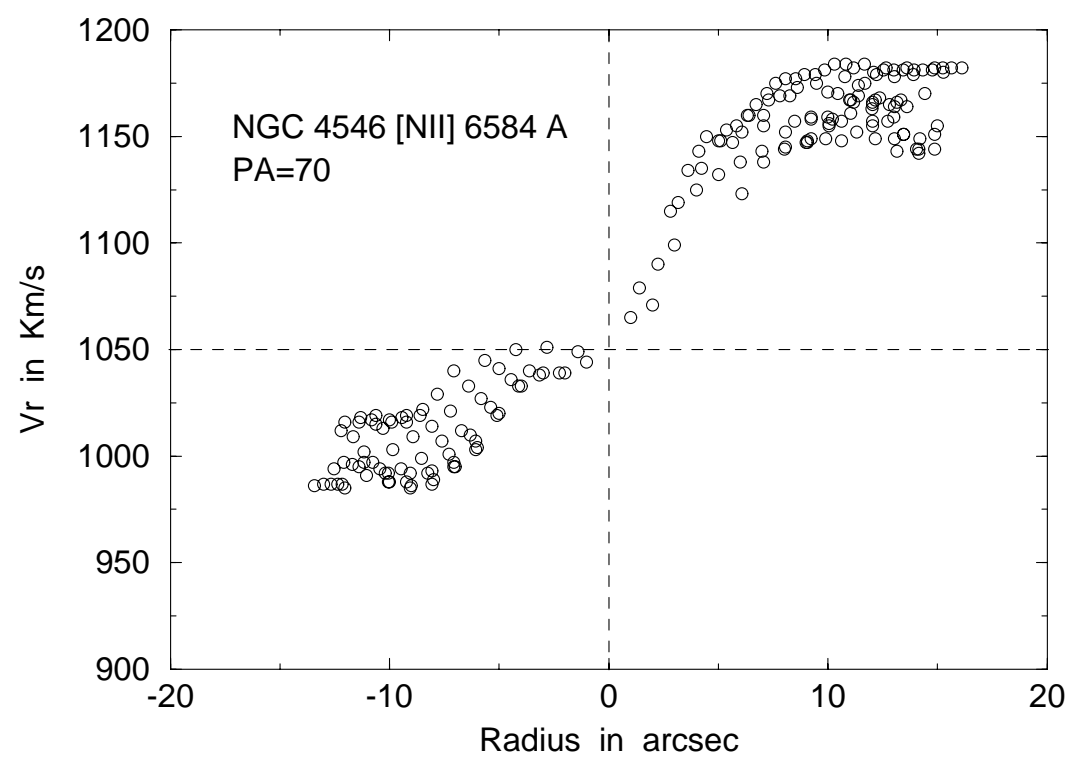

Fig. 21. NGC 4546: Line of sight velocity diagram
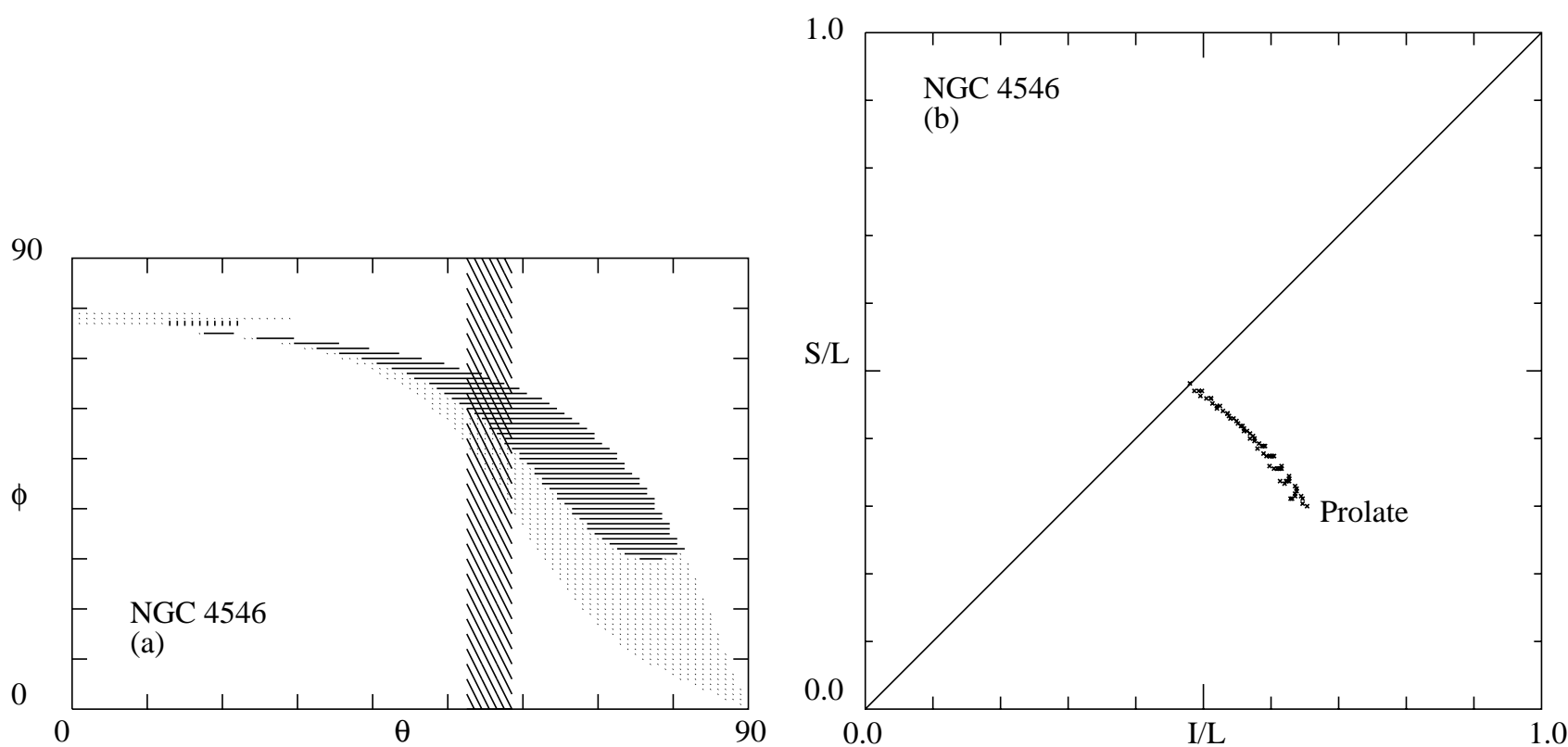

Fig. 22. NGC 4546: a) Possible viewing angles in the $\theta-\phi$ plane. b) Possible axis ratios for the ellipsoid $(S=$ short, $I=$ intermediate, $L=$ long) in accordance of $\theta$ values of showed in a) 

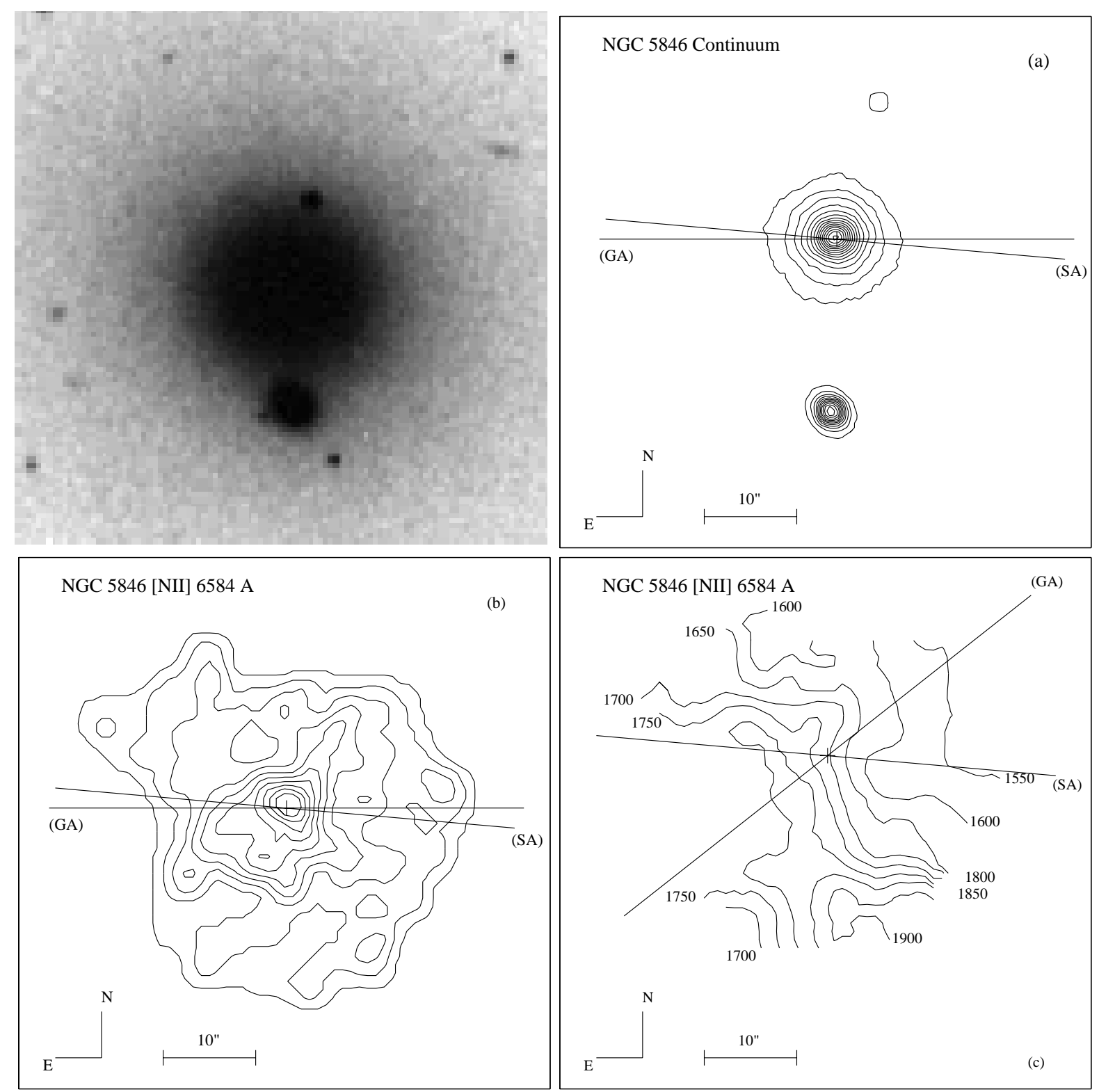

Fig. 23. NGC 5846: Field image from ESO Sky Survey, total size is 3 arcmin. a) Continuum image from our observations. b) [NII] $6584 \AA$ monochromatic map. Contours are in units of $10^{-17} \mathrm{erg} \mathrm{s}^{-1} \mathrm{~cm}^{-2} \operatorname{arcsec}^{-2}$. Lowest 7.2 and step 9.64. c) Velocity field. SA represents the stellar axis, GA represents the gas axis 


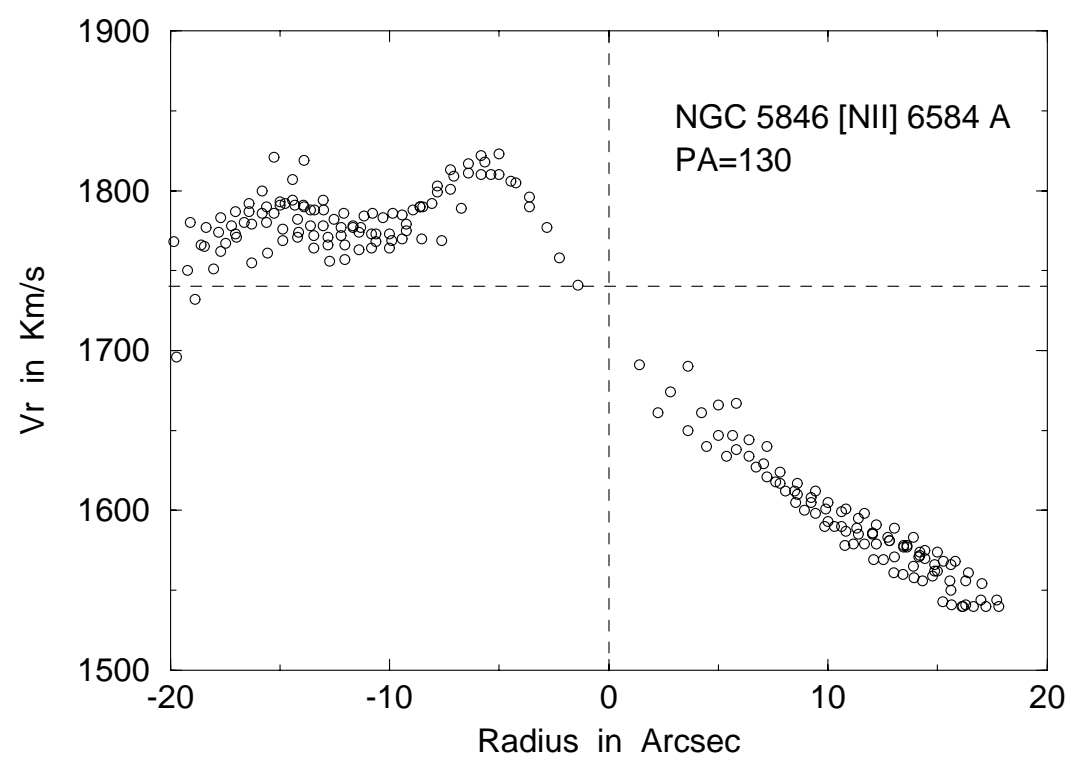

Fig. 24. NGC 5846: Line of sight velocity diagram
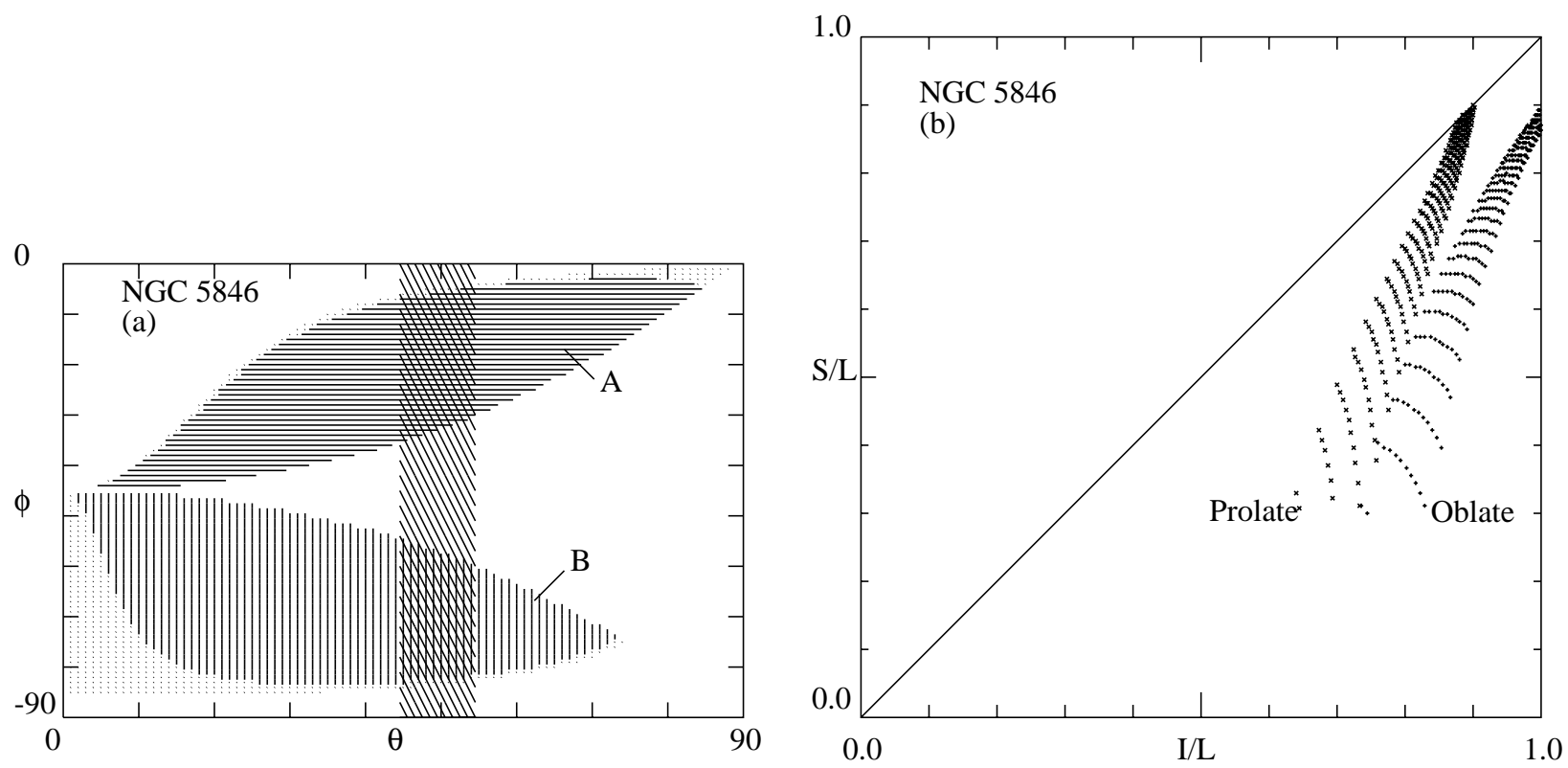

Fig. 25. NGC 5846: a) Possible viewing angles in the $\theta-\phi$ plane. Case A: gas $\perp$ short axis. Case B gas $\perp$ long axis. b) Possible axis ratios for the ellipsoid ( $S=$ short, $I=$ intermediate, $L=$ long) in accordance of $\theta$ values of showed in a) 


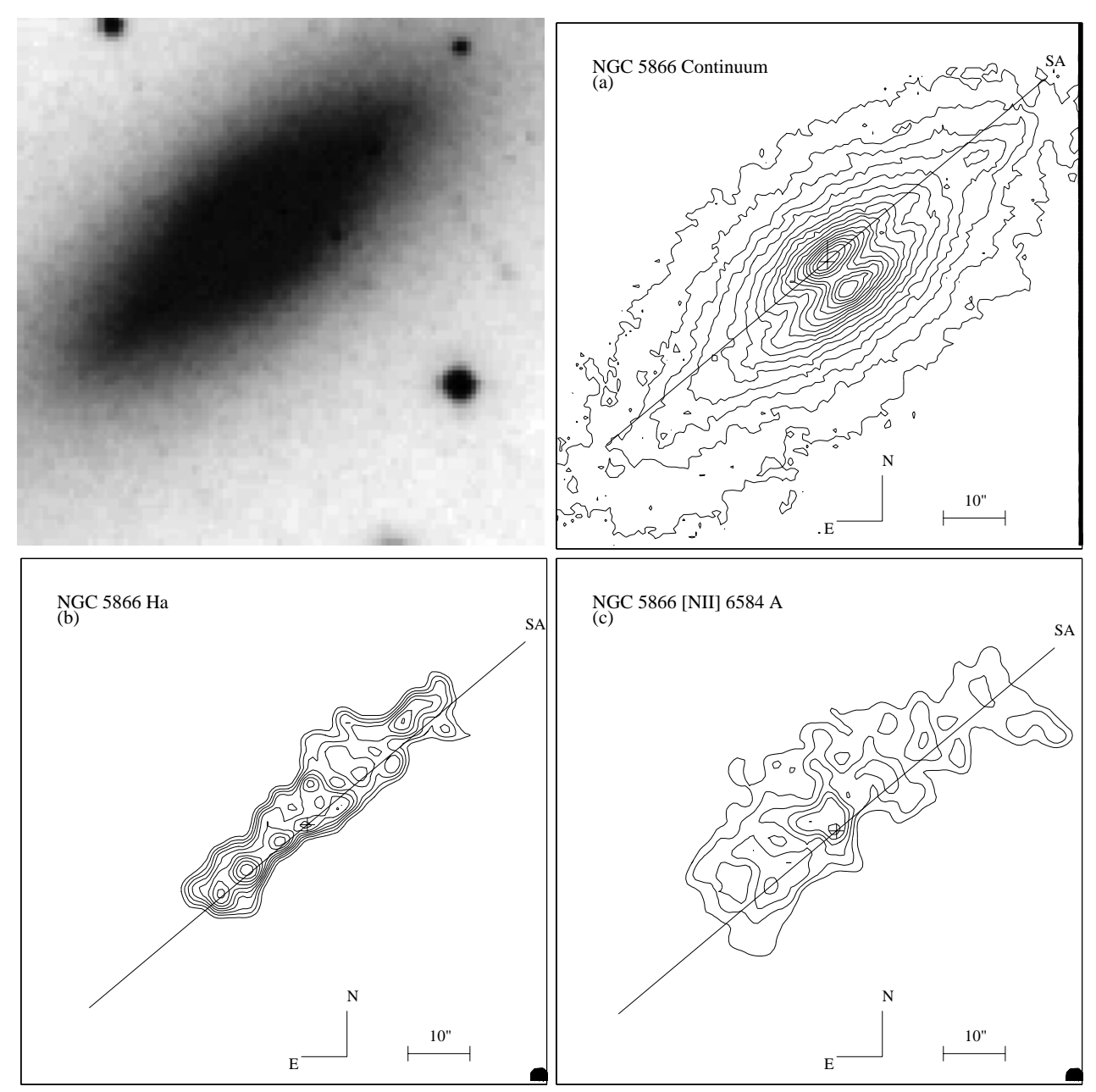

Fig. 26. NGC 5866: Field image from ESO Sky Survey, total size is 3 arcmin. a) Continuum image from our observations. b) $\mathrm{H}_{\alpha}$ monochromatic map. Contours are in units of $10^{-17} \mathrm{erg} \mathrm{s}^{-1} \mathrm{~cm}^{-2} \operatorname{arcsec}^{-2}$. Lowest 7.3 and step 7.3 . (c) [NII] $6584 \AA$ monochromatic map. Contours are in units of $10^{-17} \mathrm{erg} \mathrm{s}^{-1} \mathrm{~cm}^{-2} \operatorname{arcsec}^{-2}$. Lowest 19.5 and step 19.5. SA represents stellar axis

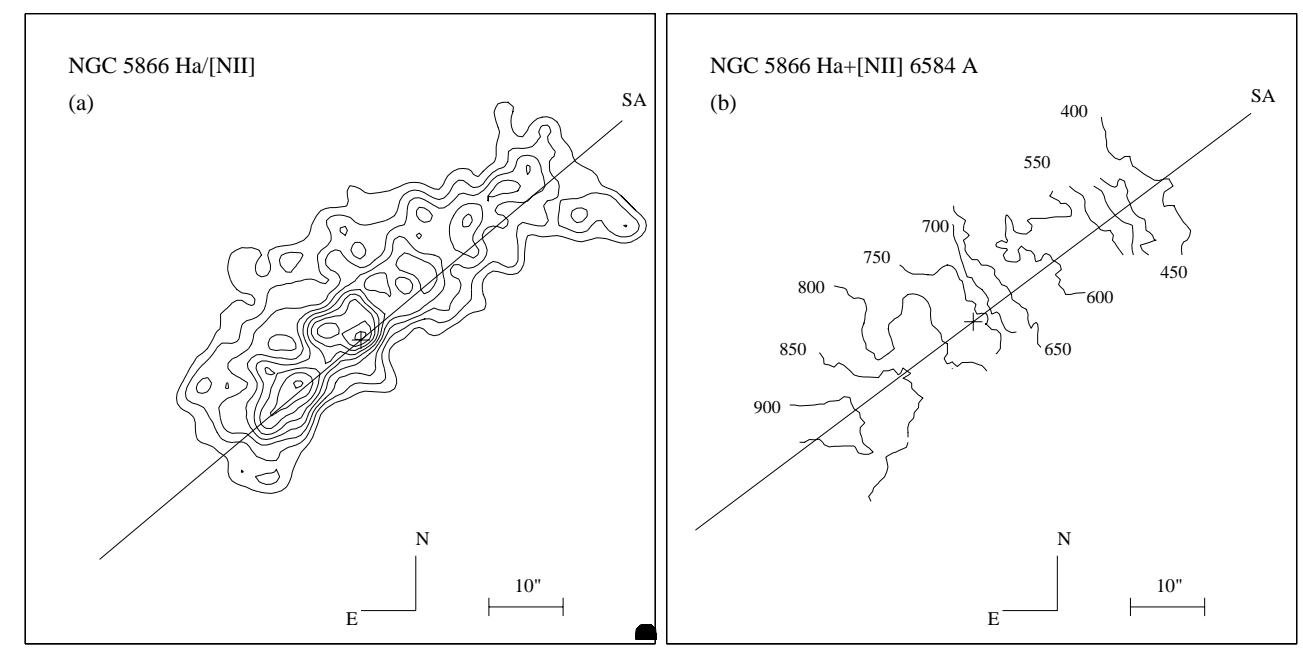

Fig. 27. NGC 5866: a) $\mathrm{H}_{\alpha} /[\mathrm{NII}]$ map, relative lowest level is 25 and the step 40. b) Velocity map of $\mathrm{H}_{\alpha}+[\mathrm{NII}]$ emission. SA is the stellar axis 

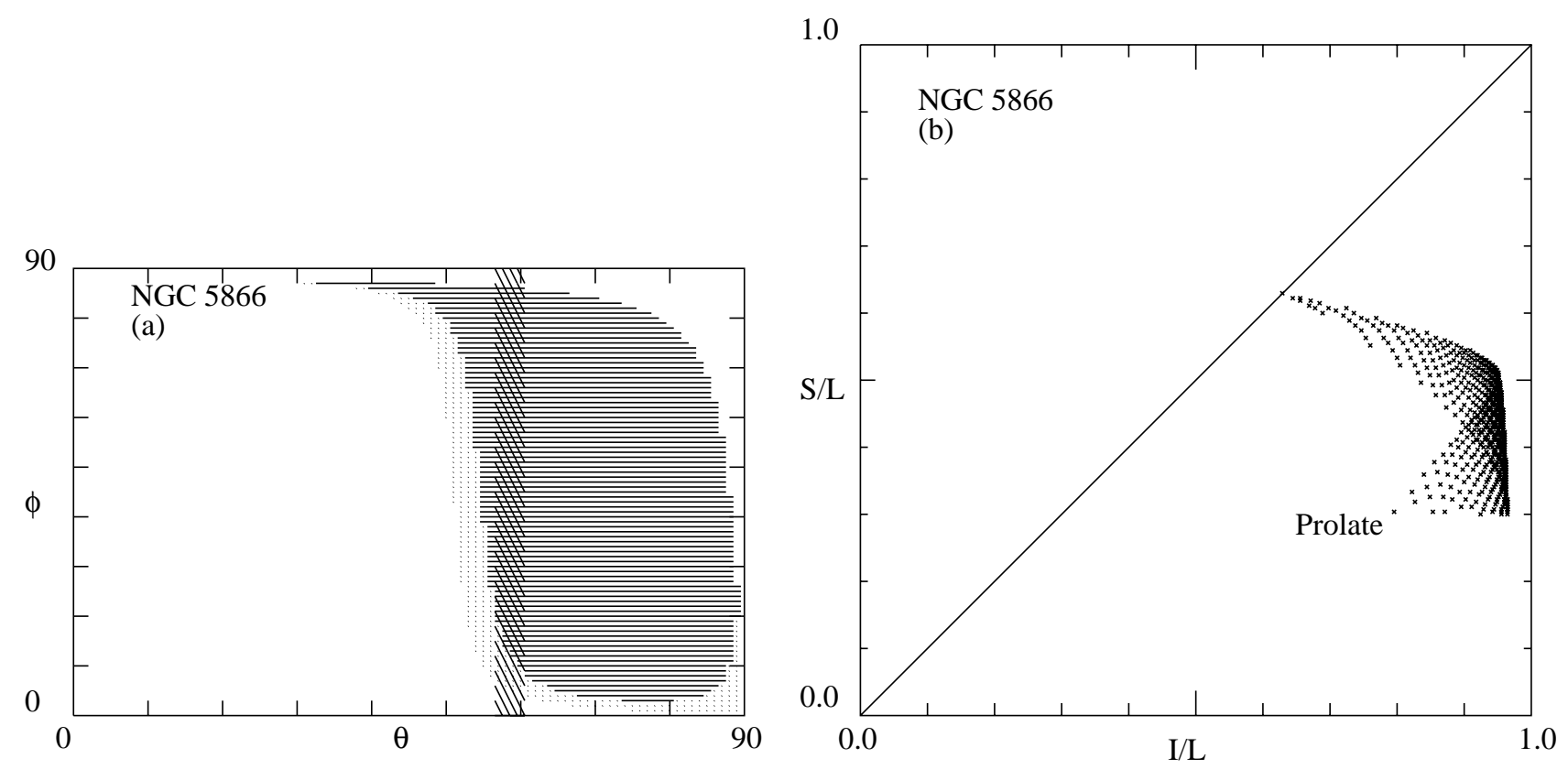

Fig. 28. NGC 5866: a) Possible viewing angles in the $\theta-\phi$ plane. b) Possible axis ratios for the ellipsoid ( $S=$ short, $I=$ intermediate, $L=$ long) in accordance of $\theta$ values of showed in a)

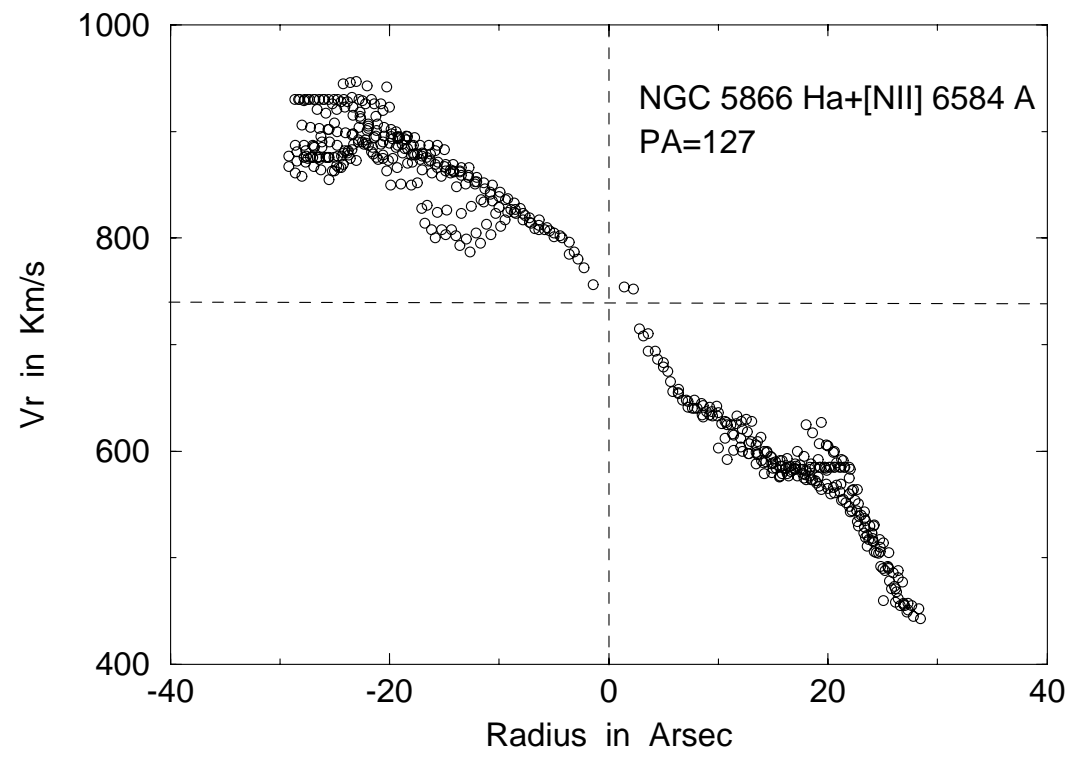

Fig. 29. NGC 5866: Line of sight velocity diagram 

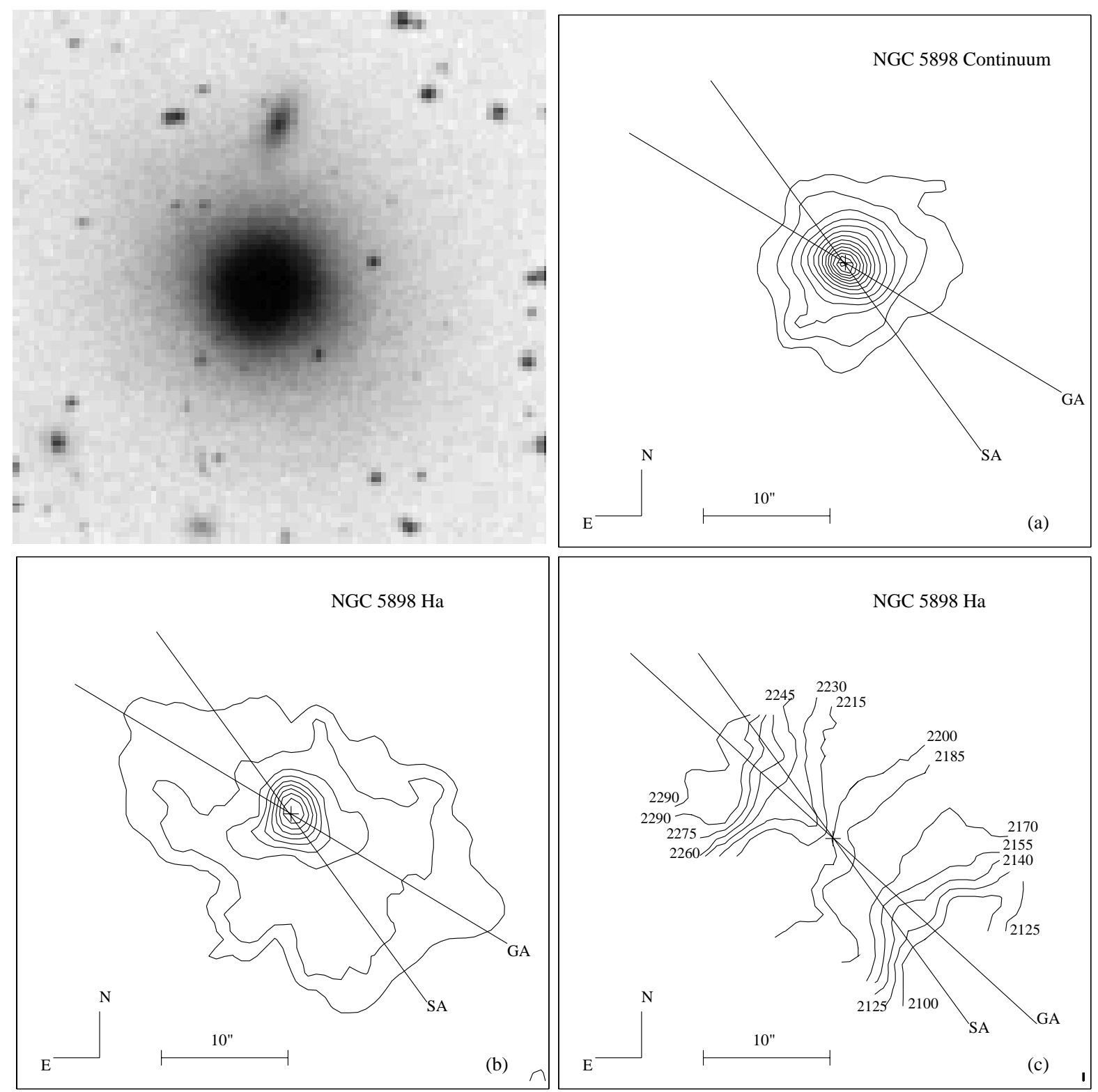

Fig. 30. NGC 5898: Field image from Digitalized Sky Survey, total size is 3 arcmin. a) Continuum image from our observations. b) $\mathrm{H}_{\alpha}$ monochromatic map. Contours are in units of $10^{-17} \mathrm{erg} \mathrm{s}^{-1} \mathrm{~cm}^{-2} \operatorname{arcsec}^{-2}$ Lowest 9.4 and step 16.4. c) Velocity field. SA represents the stellar axis, GA represents the gas axis 


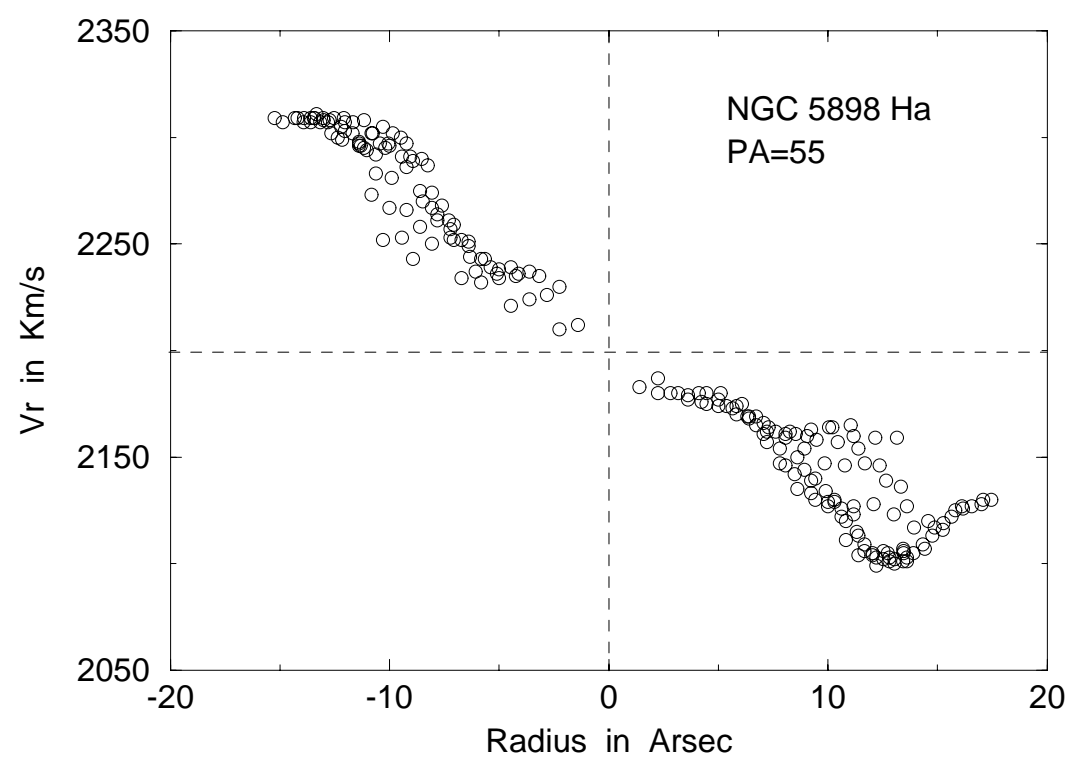

Fig. 31. NGC 5898: Line of sight velocity diagram
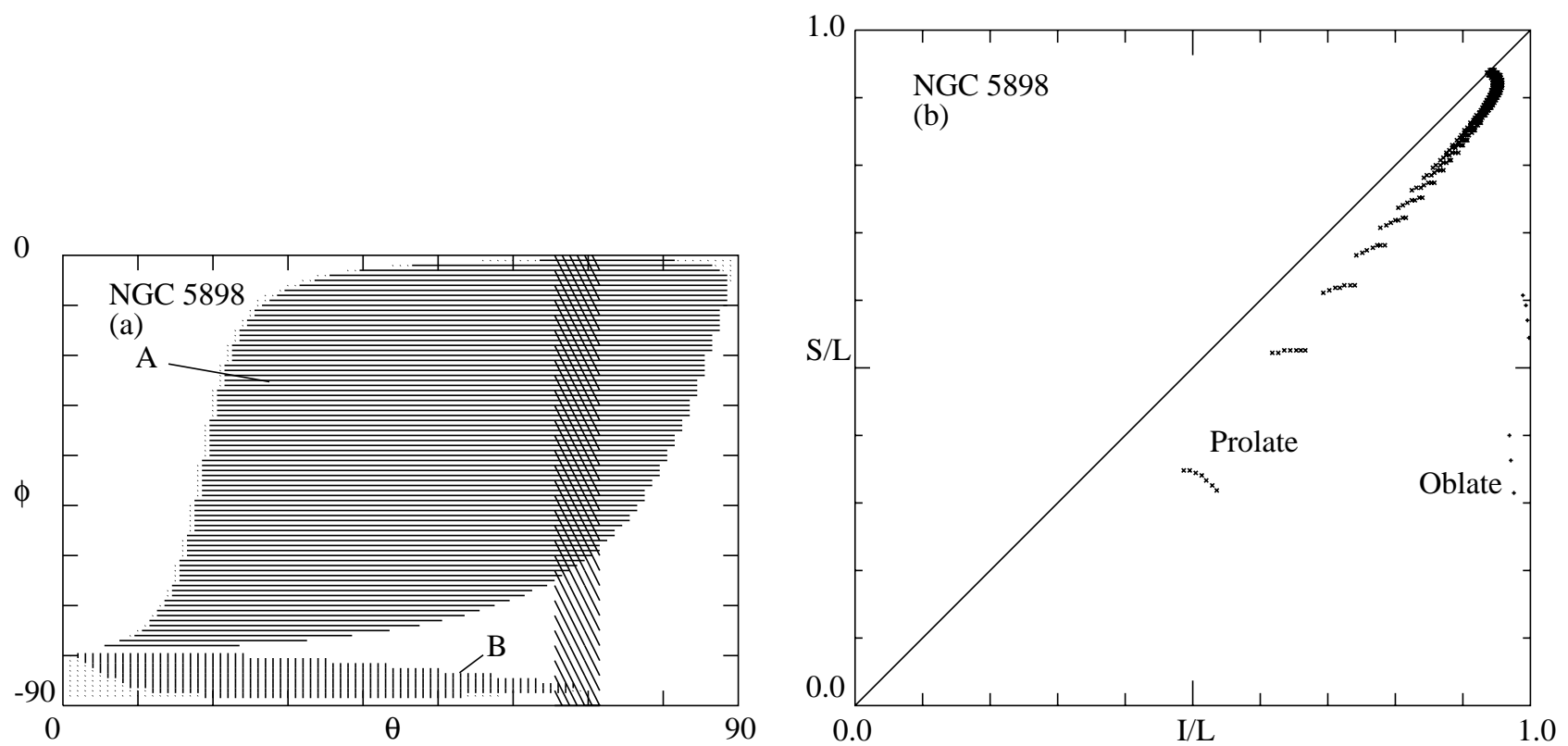

Fig. 32. NGC 5898: a) Possible viewing angles in the $\theta-\phi$ plane. Case A: gas $\perp$ short axis. Case B gas $\perp$ long axis. b) Possible axis ratios for the ellipsoid ( $S=\operatorname{short,~} I=$ intermediate, $L=\operatorname{long}$ ) in accordance of $\theta$ values of showed in a) 

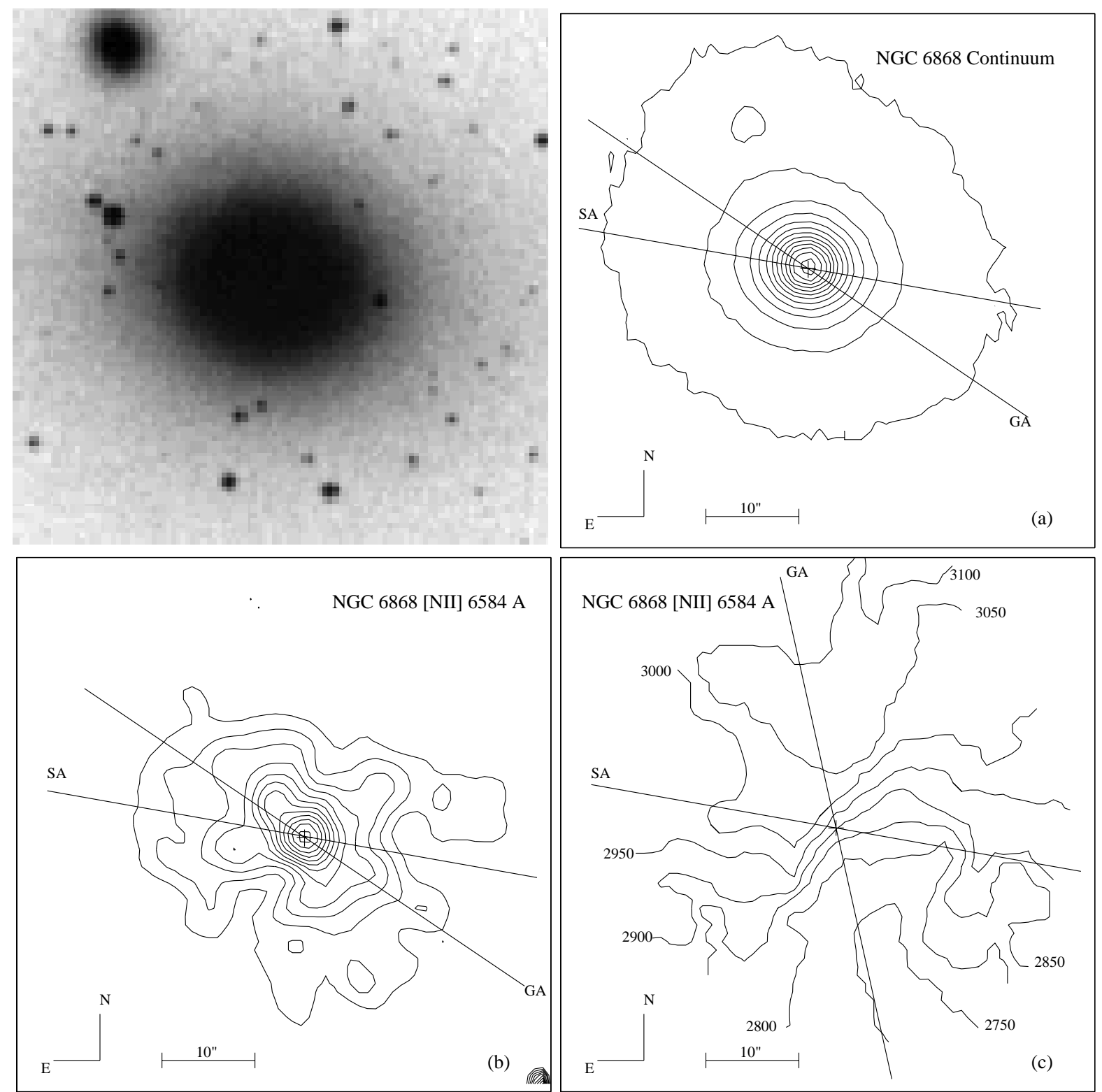

Fig. 33. NGC 6868: Field image from Digitalized Sky Survey, total size is 3 arcmin. a) Continuum image from our observations. b) [NII] $6584 \AA$ monochromatic map. Contours are in units of $10^{-17} \mathrm{erg} \mathrm{s}^{-1} \mathrm{~cm}^{-2} \operatorname{arcsec}^{-2}$. Lowest 10.0 and step 5 . c) Velocity field. SA represents the stellar axis, GA represents the gas axis 


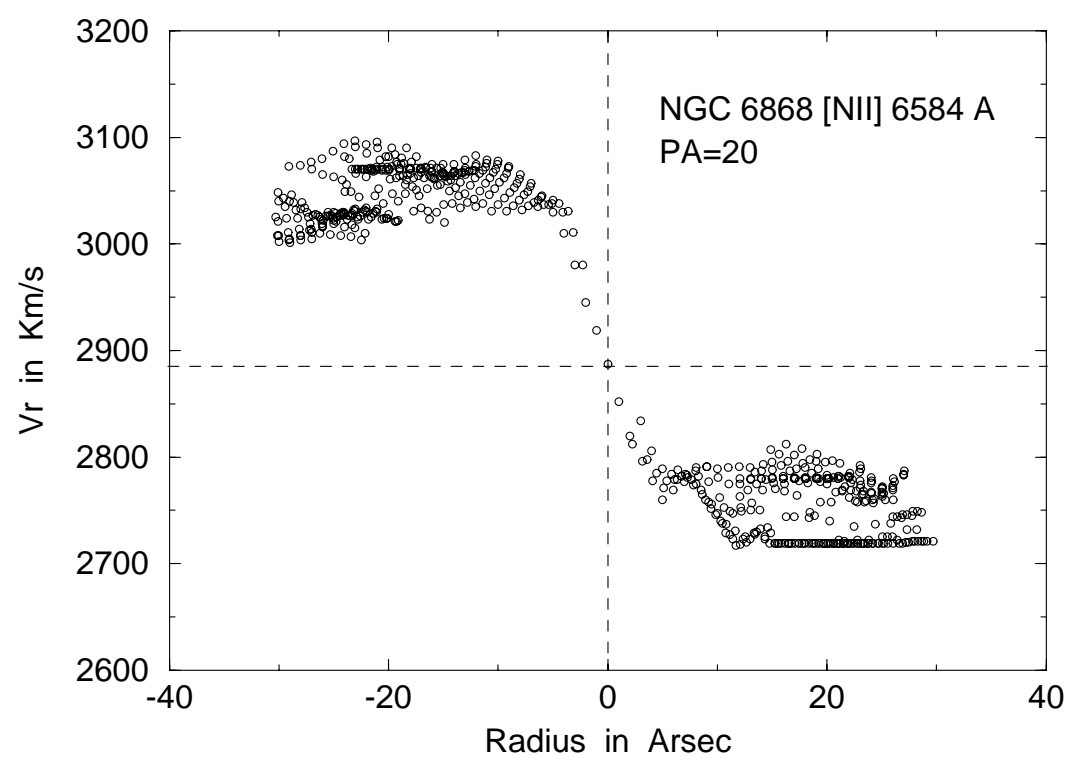

Fig. 34. NGC 6868: Line of sight velocity diagram
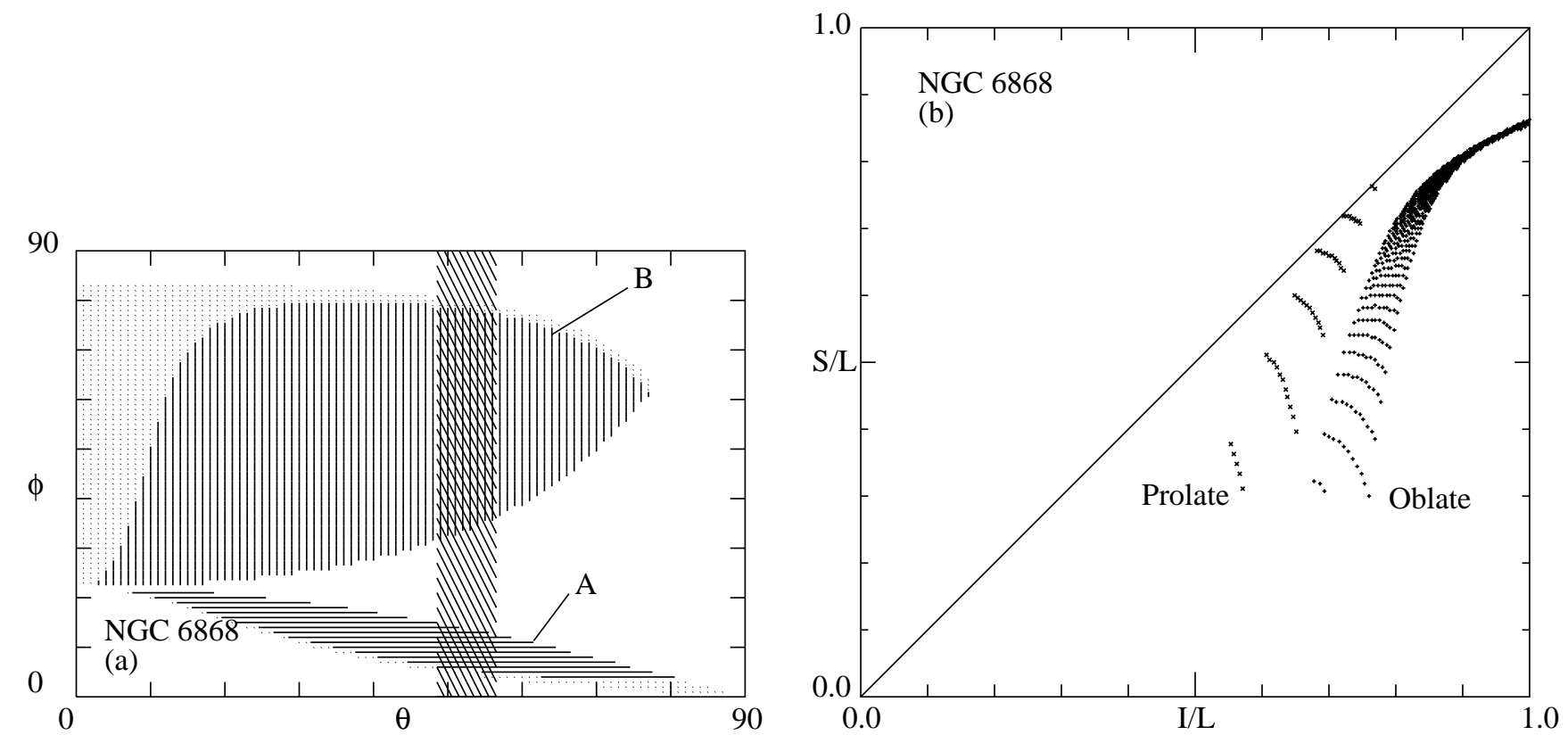

Fig. 35. NGC 6868: a) Possible viewing angles in the $\theta-\phi$ plane. Case A: gas $\perp$ short axis. Case B gas $\perp$ long axis. b) Possible axis ratios for the ellipsoid ( $S=$ short, $I=$ intermediate, $L=$ long) in accordance of $\theta$ values of showed in a) 

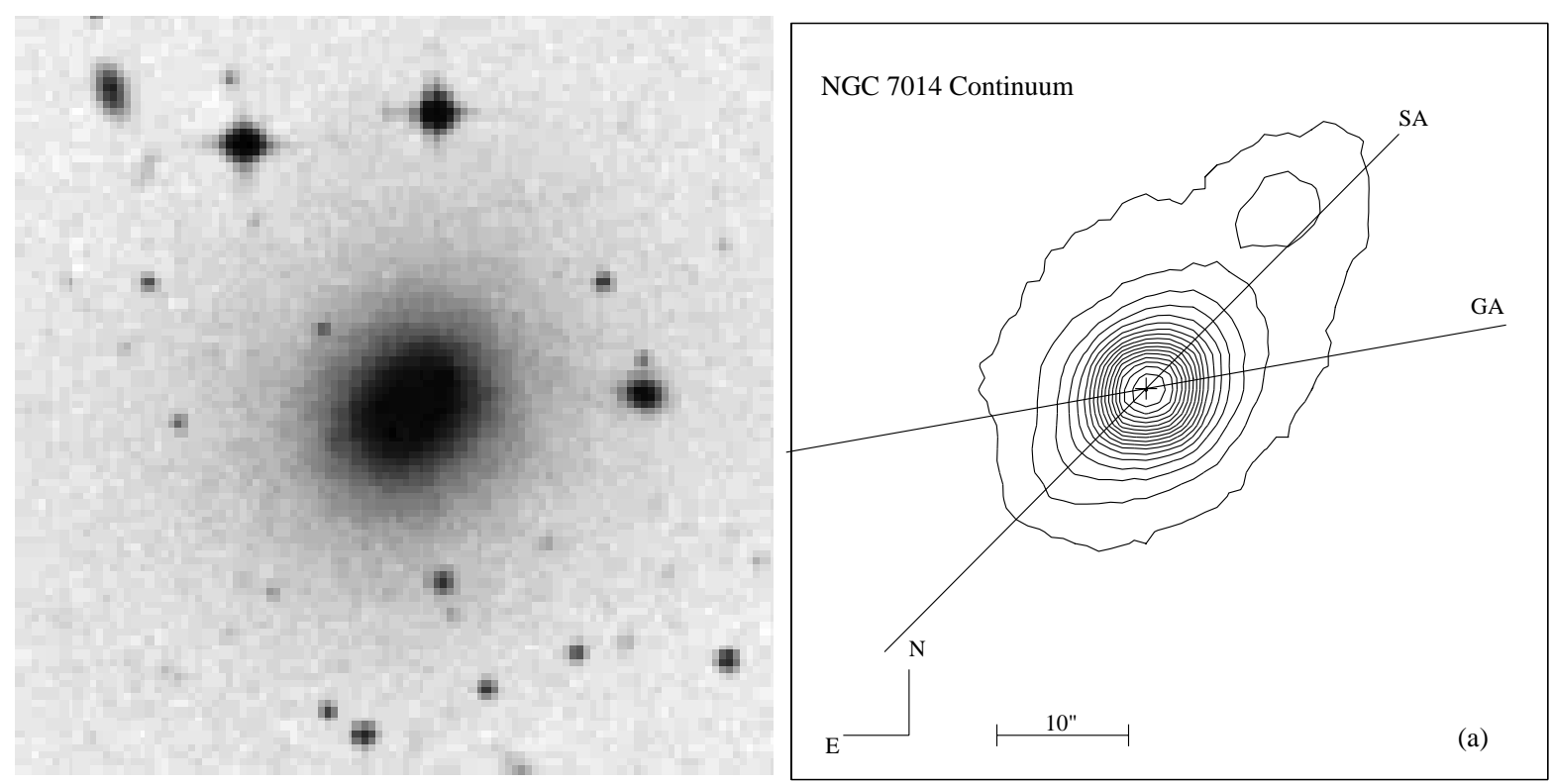

NGC 7014 [NII] 6584 A

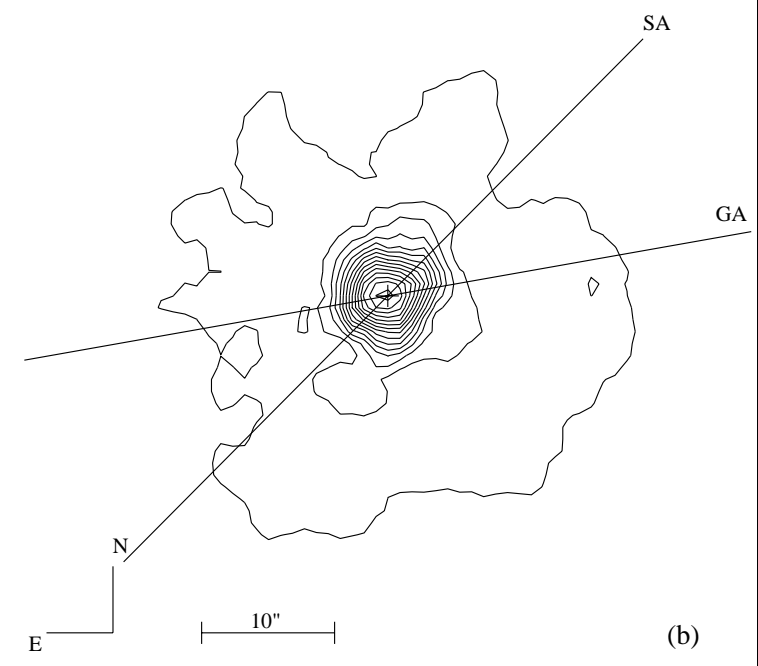

NGC 7014 [NII] 6584 A

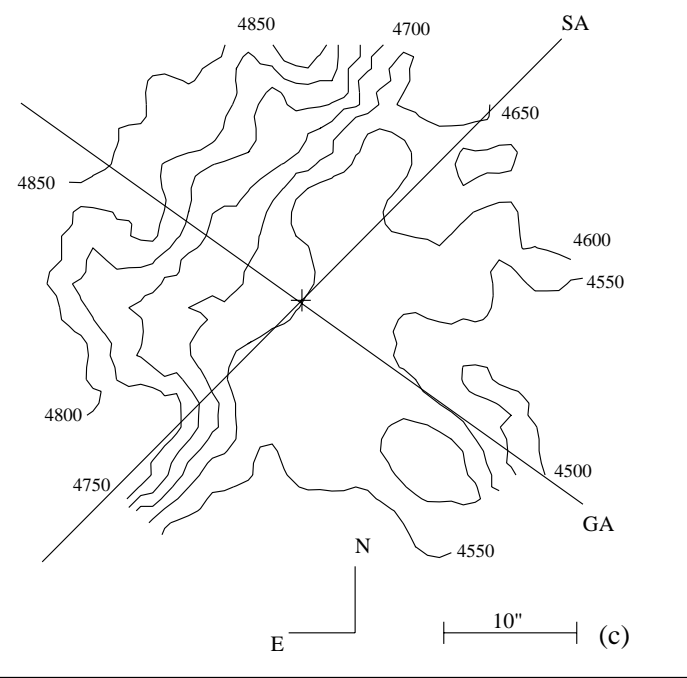

Fig. 36. NGC 7014: Field image from Digitalized Sky Survey, total size is 3 arcmin. a) Continuum image from our observations. b) [NII] $6584 \AA$ monochromatic map. Contours are in units of $10^{-17} \mathrm{erg} \mathrm{s}^{-1} \mathrm{~cm}^{-2} \operatorname{arcsec}^{-2}$. Lowest 7.5 and step 15 . c) Velocity field. SA represents the stellar axis, GA represents the gas axis 


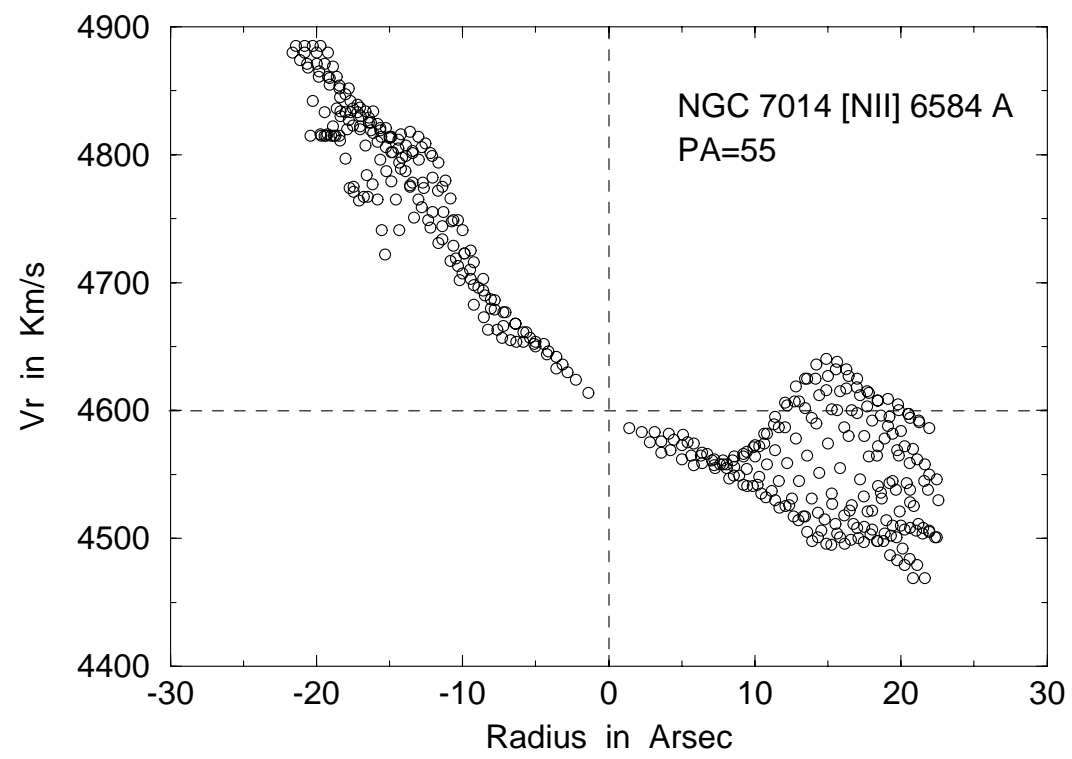

Fig. 37. NGC 7014: Line of sight velocity diagram
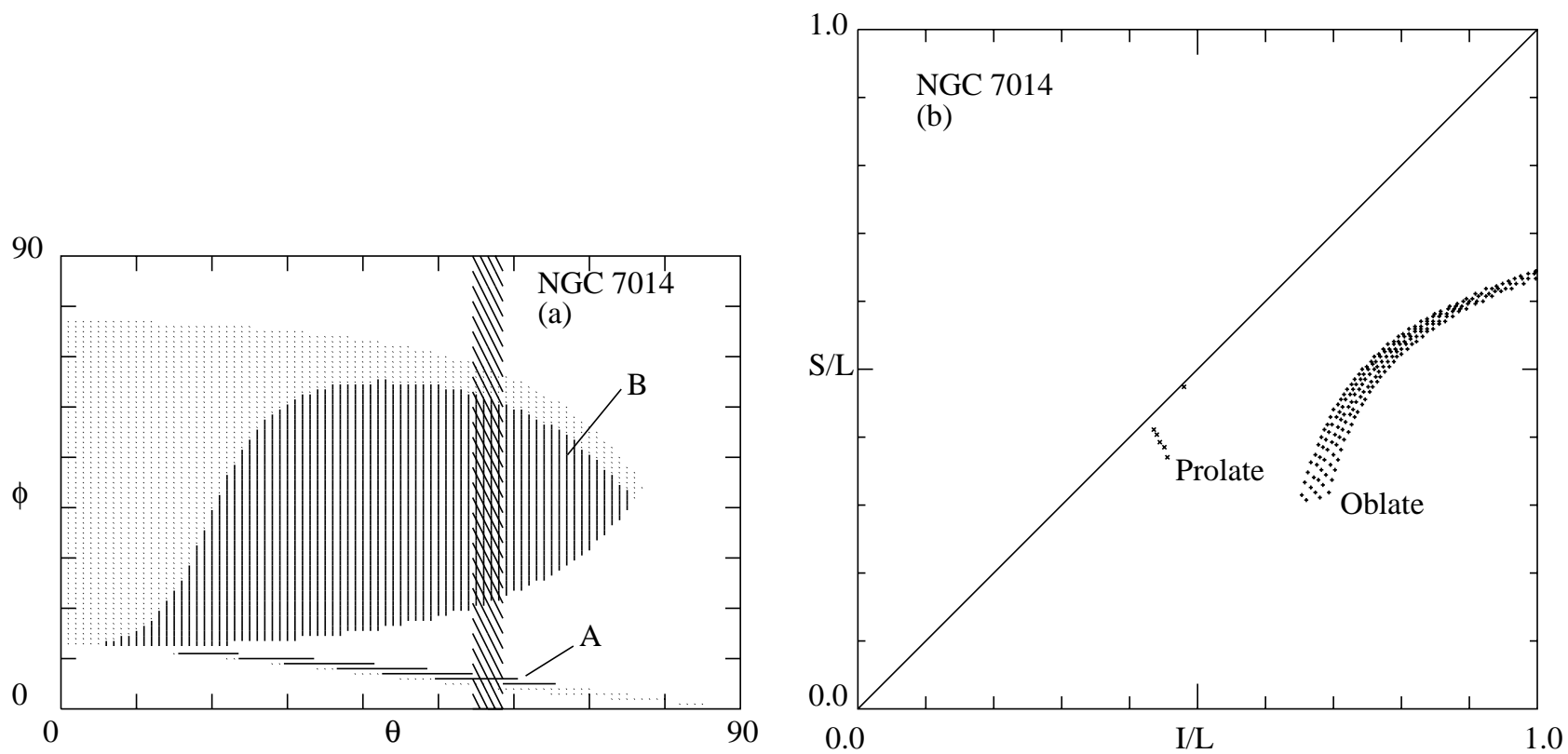

Fig. 38. NGC 7014: a) Possible viewing angles in the $\theta-\phi$ plane. Case A: gas $\perp$ short axis. Case B gas $\perp$ long axis. b) Possible axis ratios for the ellipsoid ( $S=\operatorname{short,~} I=$ intermediate, $L=\operatorname{long}$ ) in accordance of $\theta$ values of showed in a) 

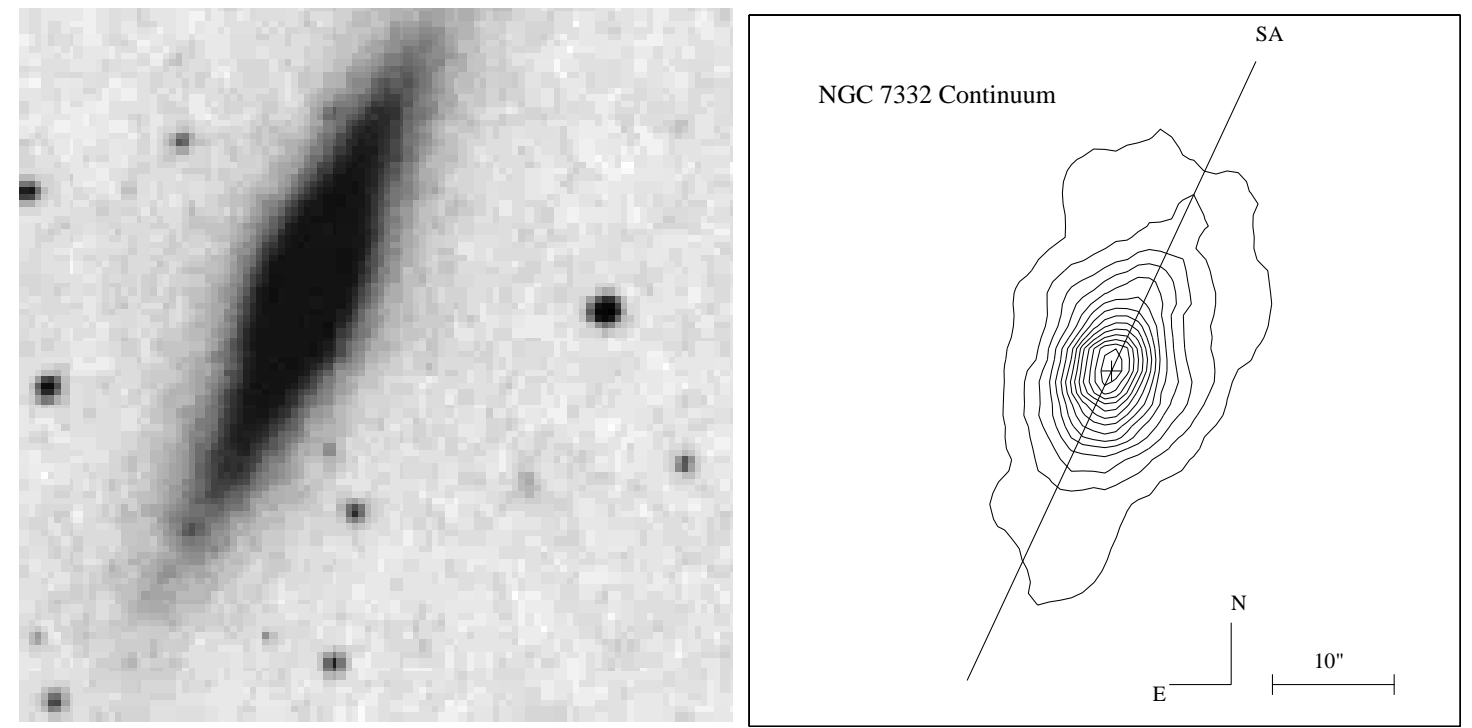

Fig. 39. NGC 7332: Same as NGC 404

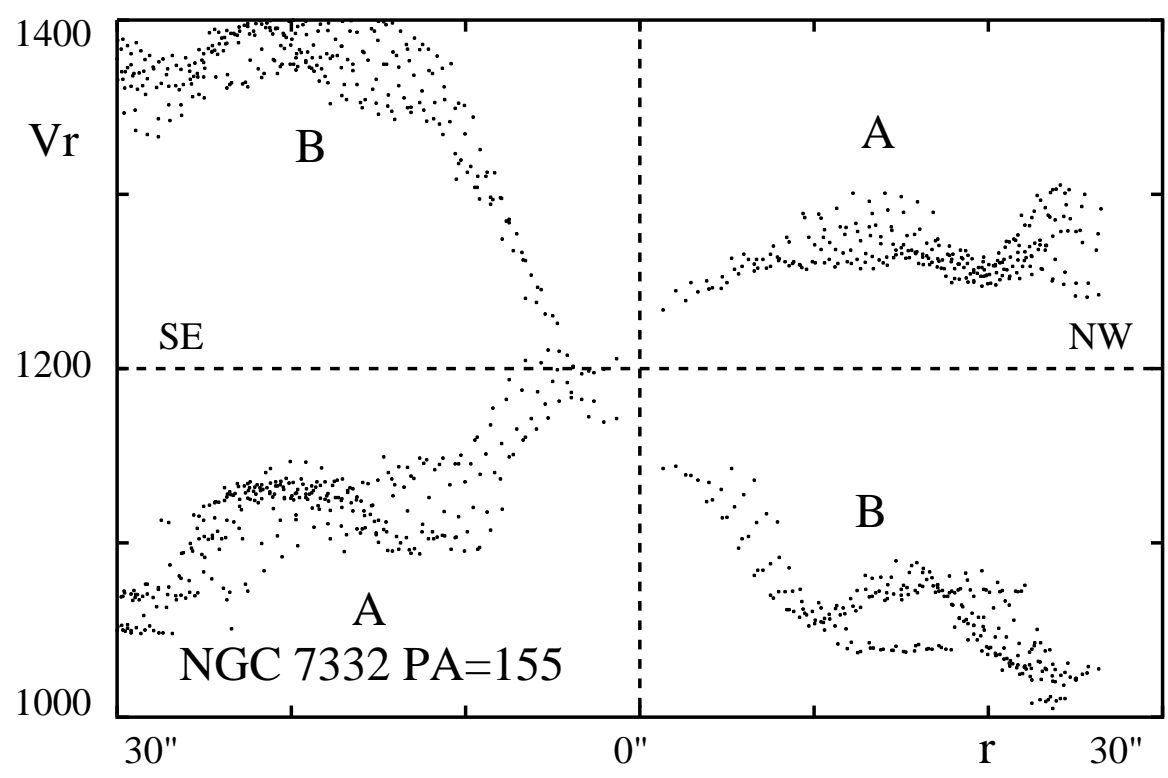

Fig. 40. NGC 7332: Line of sight velocity diagram of the two gaseous components. A: Major component. B: Second component 

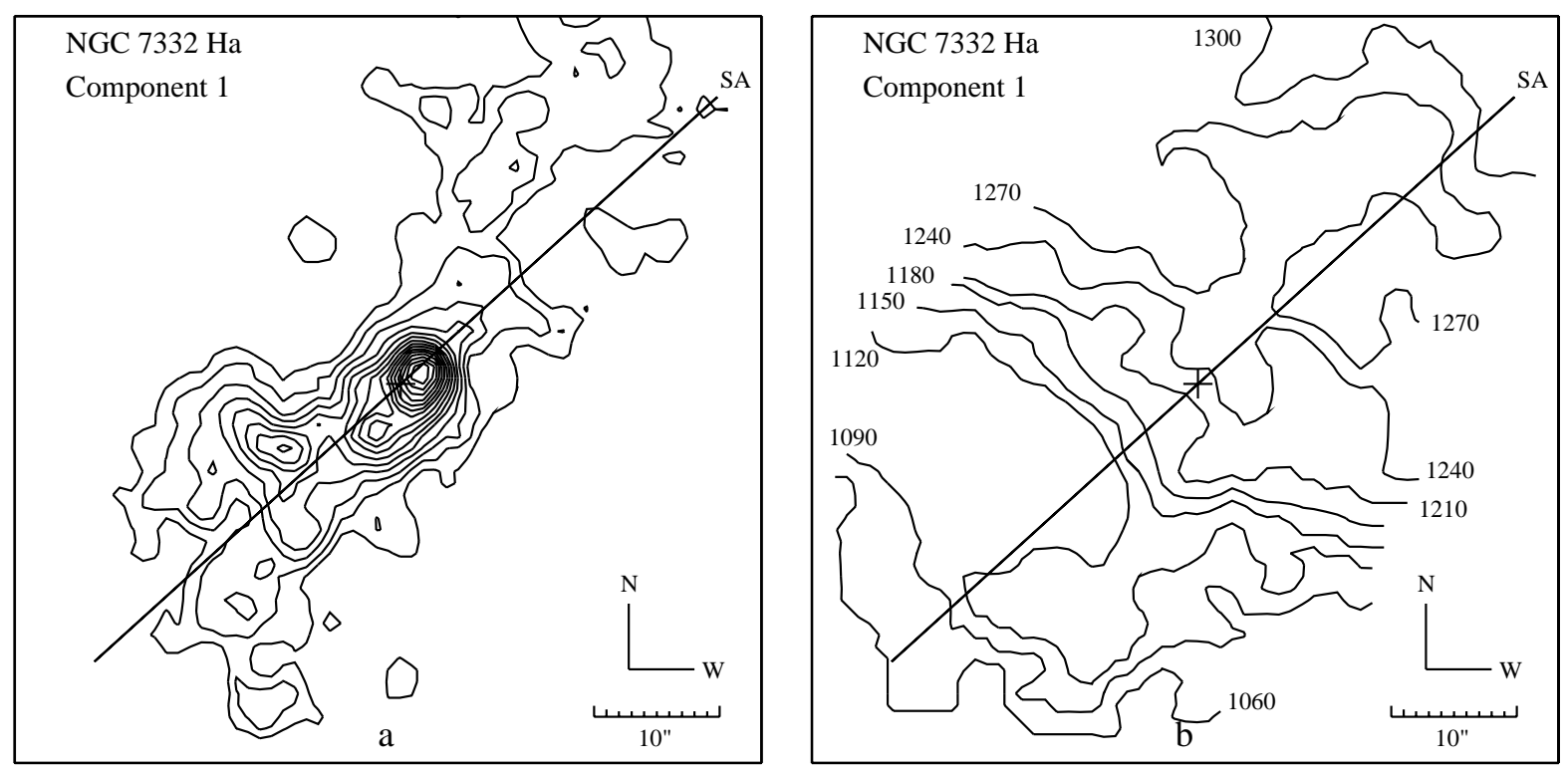

Fig. 41. NGC 7332 Component \#1: a) $\mathrm{H}_{\alpha}$ Monochromatic map. Contours are in units of $10^{-17} \mathrm{erg} \mathrm{s}^{-1} \mathrm{~cm}^{-2} \operatorname{arcsec}^{-2}$. Lowest 3 and step 7. b) Velocity map. SA is the stellar axis and GA the gas axis (which is different for velocity and monochromatic maps)
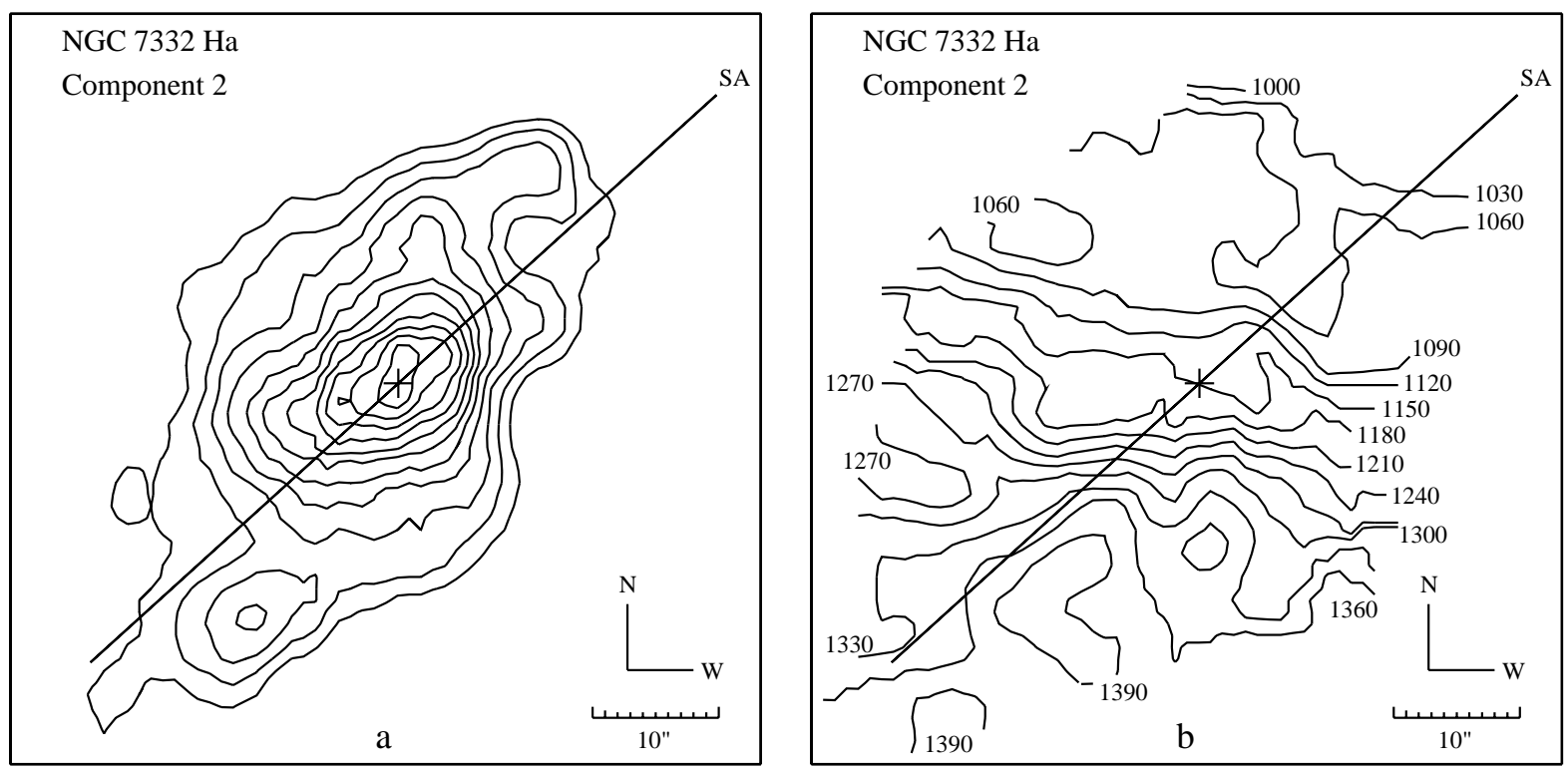

Fig. 42. NGC 7332 Component \#2: a) [NII] $6584 \AA$ Monochromatic map. Contours are in units of $10^{-17} \mathrm{erg} \mathrm{s}^{-1} \mathrm{~cm}^{-2}$ $\operatorname{arcsec}^{-2}$. Lowest 3 and step 7. b) Velocity map. SA is the stellar axis and GA the gas axis (which is different for velocity and monochromatic maps) 


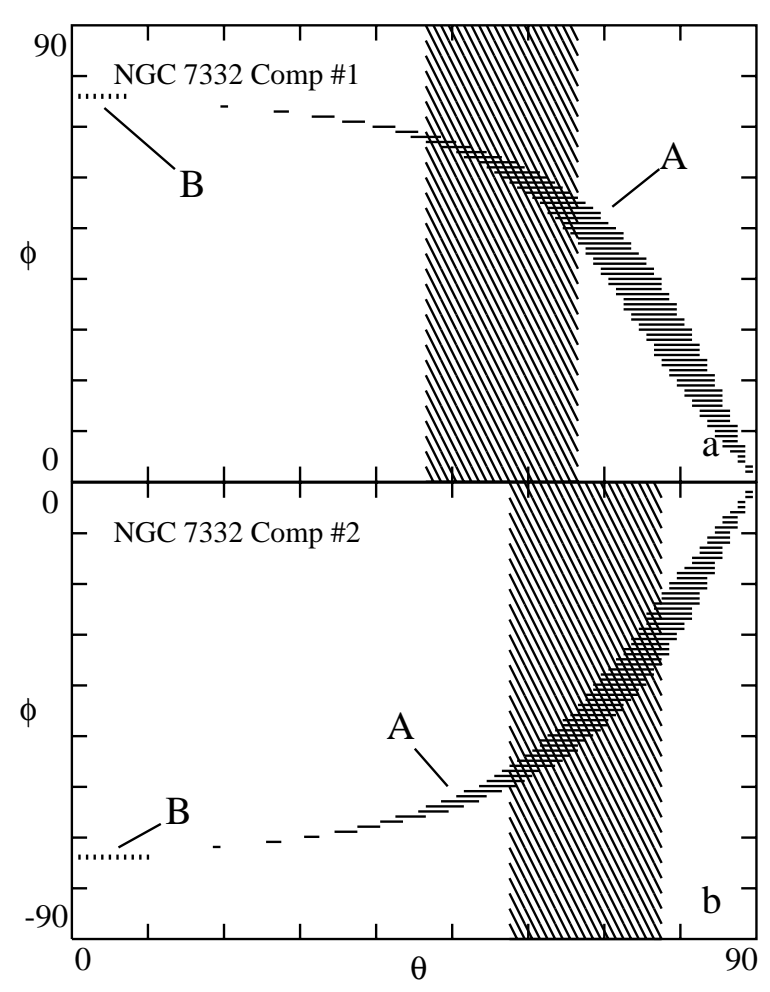

Fig. 43. NGC 7332: Possible viewing angles for component $\# 1$ a) and component \#2 b). Case A: gas $\perp$ short axis. Case B gas $\perp$ long axis

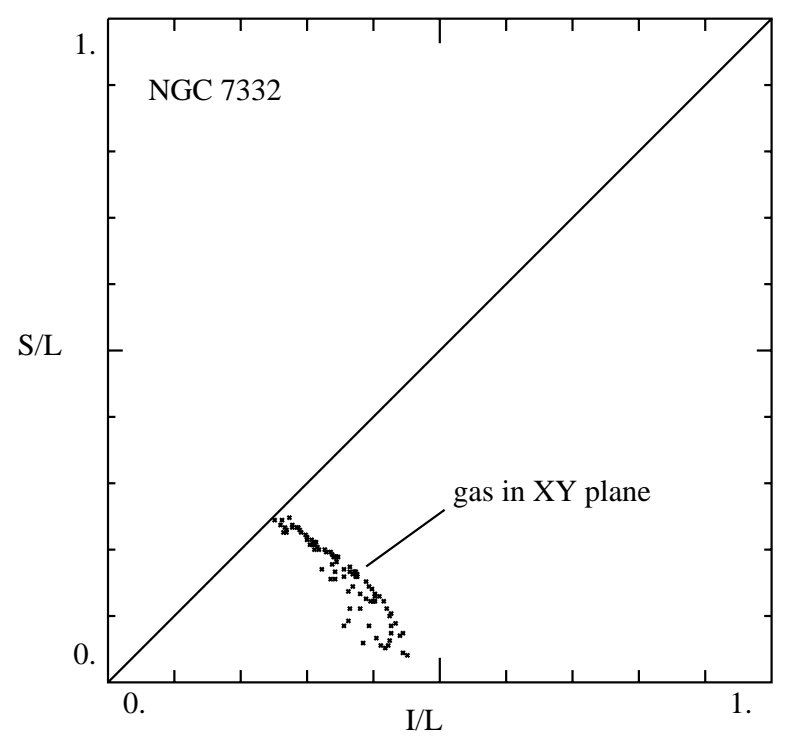

Fig. 44. NGC 7332: Possible axis ratios for component \#1 hypothesis of $5^{\circ}<\phi<15^{\circ}$
Then monochromatic maps are obtained by integrating the monochromatic profile of each component. Velocity maps are computed from the position of the emission line. For interferometer \#1, some uncertainty remains, due to the size of the smallest free spectral range and the possible confusion of the orders. Ambiguity is resolved by using other observations (interferometer \#2, previous long slit or HI observations).

Monochromatic maps have a one pixel resolution in the center of the galaxies. Spectral profiles have been binned in outer parts (to $5 \times 5$ pixels) in order to get a sufficient signal to noise ratio to measure and decompose components.

\section{Presentation of the work}

From Fig. 1 to Fig. 44, we present results of observations. For each galaxy we give a DSS image, a continuum image, a monochromatic map, a velocity field, a diagram of possible viewing angles, a graph of derived ellipsoid axis ratios and a line of sight velocity curve.

For NGC 404, we have 3 monochromatic maps $\left(\mathrm{H}_{\alpha},[\mathrm{NII}]\right.$ $6584 \AA$ and [OIII] $5007 \AA$ ). $\mathrm{A} \mathrm{H}_{\alpha} /[\mathrm{NII}]$ map is also available.

For NGC $5866 \mathrm{H}_{\alpha}$ and [NII] $6584 \AA$ maps are given. $\mathrm{H}_{\alpha} /[\mathrm{NII}]$ map was also derived.

Table 3 shows main properties of galaxy sample.

\subsection{Geometrical and dynamical aspects}

The two dimensional velocity field allows us to derive the true shape of the ellipsoid. Gas disks can be located in 2 preferred planes $X Y$ and $Y Z$ (Heiligman \& Scharzschild 1979; Tholine et al. 1982). Using De Zeeuw \& Franx (1989) model it has been possible to derive viewing angles $\theta$ and $\phi$ and possible axis ratios $I / L$ and $S / L$ with $S$ for Short axis, $I$ for Intermediate axis and $L$ for Long axis (Bertola et al. 1991; Plana \& Boulesteix 1996). Possible viewing angles and axis ratios are presented on $\theta-\phi$ plane and $I / L-S / L$ plane with hatched regions. To summarize, $\theta$ angle represents the inclination angle of the gas disk with respect to the plane of the sky. It is derived from velocity map, fitting a circular motion model to deduce the $\theta$ angle. And $\phi$ angle is the orientation of the gas disk in the triaxial galaxy referential. Inclination value and uncertainties are visualized by a vertical hatched band on $\phi-\theta$ diagrams.

Table 4 summarizes geometrical informations coming from monochromatic and velocity map. Inclination and MA angles can be different for monochromatic and velocity maps. Gas ellipticities were estimated from isophotes where signal to noise ratio is at least 10 and distance to the center is sufficent to avoid any seeing effect. More precisely:

NGC $4044^{\prime \prime}$ to $10^{\prime \prime}$ i.e. 0.14 to 0.36 effective radius $\left(r_{\mathrm{e}}\right)$; NGC $7087^{\prime \prime}$ to $14^{\prime \prime}$ i.e. 0.1 to $0.2 r_{\mathrm{e}}$ 
NGC $105210^{\prime \prime}$ to $20^{\prime \prime}$ i.e. 0.28 to $0.56 r_{\mathrm{e}}$ NGC $297416^{\prime \prime}$ to $20^{\prime \prime}$ i.e. 0.66 to $0.8 r_{\mathrm{e}}$ NGC $45467^{\prime \prime}$ to $12^{\prime \prime}$ i.e. 0.26 to $0.44 r_{\mathrm{e}}$ NGC $58467^{\prime \prime}$ to $12^{\prime \prime}$ i.e. 0.26 to $0.44 r_{\mathrm{e}}$ NGC $589814^{\prime \prime}$ to $18^{\prime \prime}$ i.e. 0.7 to $0.9 r_{\mathrm{e}}$ NGC $686812^{\prime \prime}$ to $17^{\prime \prime}$ i.e. 0.5 to $0.51 r_{\mathrm{e}}$ NGC $701418^{\prime \prime}$ to $22^{\prime \prime}$ i.e. 1.2 to $1.46 r_{\mathrm{e}}$ NGC $733217^{\prime \prime}$ to $22^{\prime \prime}$ i.e. 1.13 to $1.46 r_{\mathrm{e}}$.

\subsection{Gas masses}

Monochromatic maps were calibrated using the literature. We also take $\mathrm{H}_{\alpha} /[\mathrm{NII}]$ ratios from articles in order to deduce $\mathrm{H}_{\alpha}$ luminosity when we have [NII] $6584 \AA$ observations. To calculate gas masses we use Case B recombination in Osterbrock 1974.

$M_{\mathrm{HII}}=2 \cdot 810^{-3} \cdot D^{2} \cdot F\left(\mathrm{H}_{\alpha}\right) \cdot n_{\mathrm{e}}$

where $M_{\mathrm{HII}}$ is expressed in $M_{\odot}, F\left(\mathrm{H}_{\alpha}\right)$ in $10^{-14} \mathrm{erg} \mathrm{cm}^{-2}$ $\mathrm{s}^{-1}, D$ in $\mathrm{Mpc}$ and $n_{\mathrm{e}}$ in $\mathrm{cm}^{-3}$.

It only has been possible to have electron density for a few objects, for the majority of the object we took the value $1000 \mathrm{~cm}^{-3}$.

Table 5 gives the different gas masses for hot, warm and cool components. $\mathrm{X}$ and radio data are from Roberts et al. (1991) except for NGC 708 for which radio luminosity is from Fanti et al. (1977). Gas masses for HI cold gas and X-ray hot gas are also from Roberts et al. (1991). Far InfraRed (FIR) luminosity has been calculated using IRAS flux at $60 \mu$ and $100 \mu$ and with the formula given in the "Cataloged galaxies and QSO observed in the IRAS survey" from Londsdale et al. (1989).

$\log (\mathrm{FIR})=\log [1.26(F 60)+F(100)]$

where $F(60)=2.58 .10^{-14} f_{\nu(60)}$ and $F(100)=10^{-14}$ $f_{\nu(100)} \cdot f_{\nu(60)}$ and $f_{\nu(100)}$ are IRAS flux density in Jansky.

In Table 5 we give the total mass of the galaxy deduced from the maximum velocity of the line of sight velocity curve (Lequeux 1983). The last column gives the ratio of the ionized gas with respect to the total mass. As we can see ionized gas represents less than $310^{-6} M_{\odot}$ for most of the galaxy sample. Exception of NGC 5898 where ionized gas represents more than $510^{-6} M_{\odot}$.

\subsection{Comments on individual objects}

\subsubsection{NGC 404}

This SO galaxy is characterized by a strong and skewed dust lane (Barbon et al. 1982). HI was detected by Baars et al. (1976), molecular gas was observed by Wiklind \& Henkel (1990). The velocity amplitude of the gas is low, $\pm 40 \mathrm{~km} \mathrm{~s}^{-1}$ and kinematical Major Axis (MA) of the gas is almost aligned with stellar MA. Figure 2 shows monochromatic images in $\mathrm{H}_{\alpha}$, [NII] $6584 \AA$ and [OIII]
$5007 \AA$ emission lines. $\mathrm{H}_{\alpha}$ and [NII] $6584 \AA$ extensions are comparable but the [OIII] $5007 \AA$ map shows a larger extension (about $30^{\prime \prime}$ ) with two distinct lobes.

The weak difference between stellar MA and gas MA explains that the region of possible $\phi$ viewing angles is very wide. The case with gas perpendicular to the short axis is not constrained and the other case with gas perpendicular to the long axis is marginal. It is very hard to conclude in what plane the gas disk is situated.

Figure $4 \mathrm{~b}$ shows $\mathrm{H}_{\alpha} /[\mathrm{NII}]$ map. It's clear that this ratio is not constant for the total galaxy. The map shows a strong gradient between East and West. The mean value is 2.13 which is consistent with the literature (Kim 1989).

\subsubsection{NGC 708}

This E2 galaxy belongs to the Abell 262 cluster and it is usually qualified as a Cooling Flow. Baars et al. 1976 have discovered $\mathrm{HI}$ in emission but no gas mass has been derived. Molecular gas was also detected by Braine et al. (1994) he gives a superior limit for the mass but this limit is very low for an object who has to accrete $300 M_{\odot} /$ year in the hypothesis of a cooling flow (Heckman et al. 1989). Observations show a double gaseous component. A monochromatic image and a velocity field have been derived for each of them. Both components are not counter rotating with respect to one another but Position Angles (PA) of the MA of both components are strongly different. The first one has a PA of the MA equal to $137^{\circ}$ and the second component has a PA of $42^{\circ}$. So the second component is almost aligned with the stellar component and the first is decoupled with it. The $\phi$ viewing angle is quite well defined for the first component but for the second one there is no constraint at all.

\subsubsection{NGC 1052}

This galaxy is a well known E2 elliptical with gaseous disk (Davies et al. 1986). A detailed description of that object has been done by Plana \& Boulesteix (1996). It has also been discovered a double gaseous component in that object.

\subsubsection{NGC 2974}

This isolated galaxy is one of the brightest objects of the sample. HI emission was discovered and an atomic gas disk has been put in evidence by Kim (1988). Ionized gas has been shown by Demoulin et al. (1984), Kim (1989) and Goudfrooij et al. (1994). Ionized gas kinematics has been studied by Bettoni (1992). This object also shows an X-ray emission (Forman et al. 1985). Contrary to most of elliptical galaxies, ionized gas and HI gas components MA of NGC 2974 are aligned with respect to stars. The $\phi$ viewing angle is not constrained at all because of the small difference between MA of gas and stars. This object is a good candidate for an internal origin of ionized gas. 
Table 4. Geometrical properties of galaxies

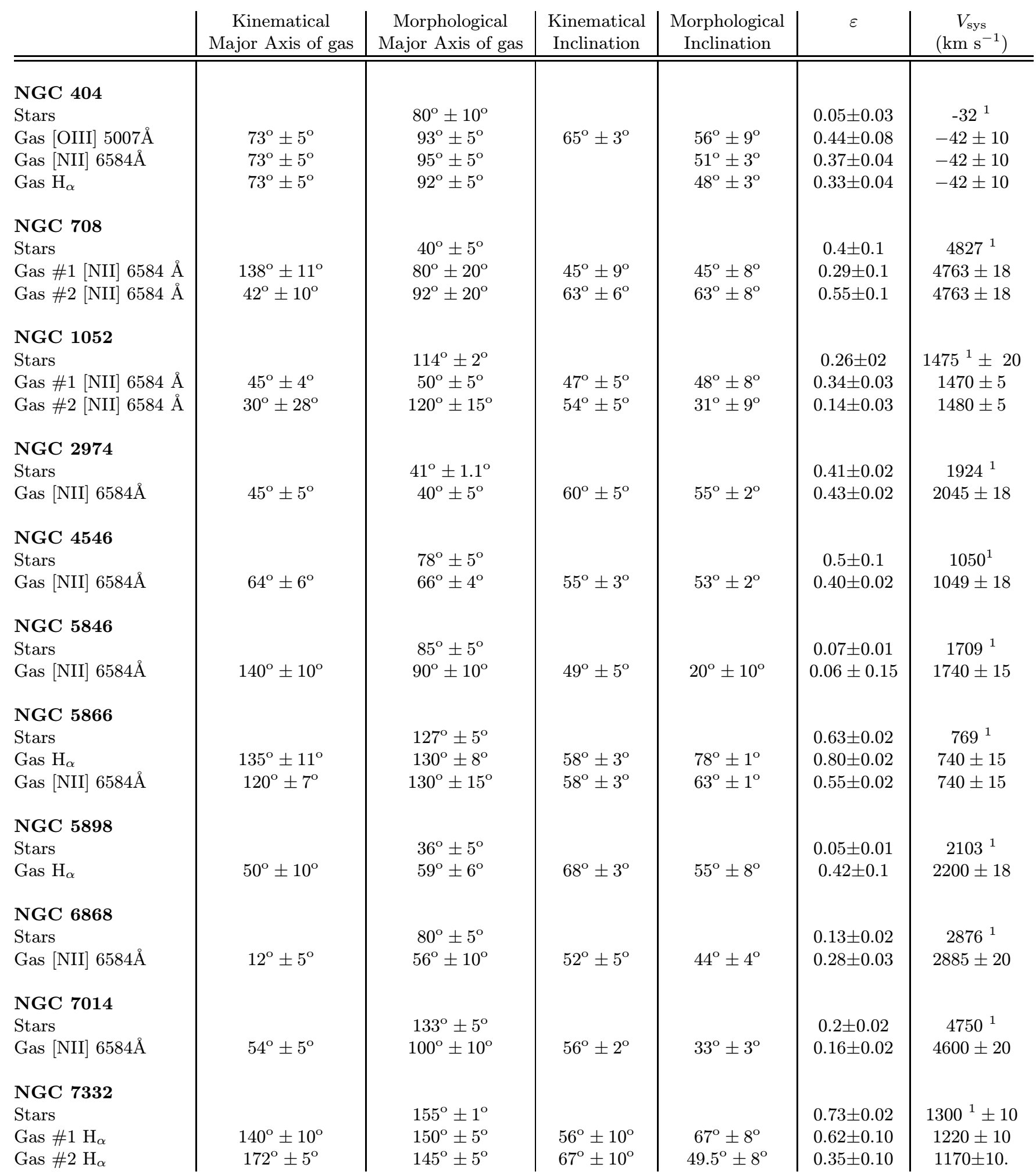

Col 1: Name.

Col 2: Gas Major Axis (MA) from velocity map.

Col 3: Gas MA from monochromatic map.

Col 4: Inclination with respect of the plane of sky calculated from velocity map.

Col 5: Inclination with respect of the plane of sky calculated from monochromatic map.

Col 6: Stellar and gas ellipticities. $\varepsilon=1-b / a$.

Col 7: Heliocentric velocity.

${ }^{1}$ Heliocentric Velocity from Faber et al. (1989). 
Table 5. Gas masses. For all objects dust mass, X hot gas mass and HI cold gas mass are from Roberts et al. (1991). X and radio luminosity are from Roberts et al. (1991) except for NGC 708 which radio luminosity is from Fanti et al. (1977)

\begin{tabular}{l|c|c|c|c|c|c|c|c}
$\begin{array}{c}D(\mathrm{Mpc}) \\
\left(H_{0}=75\right)\end{array}$ & $M_{\mathrm{B}}$ & $\begin{array}{c}\log L(\mathrm{FIR}) \\
\mathrm{erg} \mathrm{s}^{-1}\end{array}$ & $\begin{array}{c}\log L(\mathrm{X}) \\
\mathrm{erg} \mathrm{s}^{-1}\end{array}$ & $\begin{array}{c}\log L(\mathrm{radio}) \\
\mathrm{erg} \mathrm{s}^{-1} \mathrm{hz}^{-1}\end{array}$ & $\begin{array}{c}\text { Dust } \\
\text { Mass } \\
10^{4} M_{\odot}\end{array}$ & $\begin{array}{c}\text { Hot Gas } \\
\text { Mass } \\
10^{8} M_{\odot}\end{array}$ & $\begin{array}{c}\text { HI } \\
\text { Mass } \\
10^{8} M_{\odot}\end{array}$ \\
\hline \hline N 404 & 10.0 & -19.8 & 42.20 & - & - & 0.89 & - & 1.20 \\
N 708 & 63.1 & -21.2 & - & - & 37.15 & - & - & - \\
N 1052 & 18.6 & -20.9 & 42.27 & 40.36 & 37.53 & 18.62 & - & 11.48 \\
N 2974 & 25.7 & -20.1 & 42.44 & 40.35 & $<36.30$ & 148.00 & 9.54 & 15.85 \\
N 4546 & 12.6 & -19.2 & 41.55 & - & 35.39 & 10.90 & - & 2.57 \\
N 5846 & 22.3 & -20.9 & $<41.34$ & 41.33 & 35.90 & $<102.30$ & 66.06 & $<0.5$ \\
N 5866 & 12.2 & -19.6 & 42.83 & 39.60 & 35.45 & 145.47 & $<22.38$ & 0.37 \\
N 5898 & 30.9 & -19.9 & 41.78 & 39.30 & $<36.41$ & $<17.49$ & $<11.75$ & $<4.78$ \\
N 6868 & 38.3 & -21.2 & 42.77 & - & - & 229.08 & - & 14.12 \\
N 7014 & 67.0 & -20.7 & 42.84 & - & - & - & - & - \\
N 7332 & 19.5 & -19.8 & 41.72 & 39.18 & $<35.65$ & $<5.12$ & $<6.45$ & $<2.88$
\end{tabular}

\begin{tabular}{l|c|c|c|c|c} 
& $\begin{array}{c}F\left(\mathrm{H}_{\alpha}\right) \\
10^{-14} \\
\mathrm{erg} \mathrm{cm}^{2} \mathrm{~s}^{-1}\end{array}$ & $\begin{array}{c}\log L\left(\mathrm{H}_{\alpha}\right) \\
\mathrm{erg} \mathrm{s}^{-1}\end{array}$ & $\begin{array}{c}\text { HII Gas } \\
\text { mass } \\
10^{4} M_{\odot}\end{array}$ & $\begin{array}{c}\text { Total } \\
\text { mass } \\
10^{10} M_{\odot}\end{array}$ & $\begin{array}{c}\text { Ionized gas/ } \\
\text { total mass } \\
10^{-6}\end{array}$ \\
\hline \hline NGC 404 & 15.0 & 39.25 & 0.4 & 0.60 & 0.67 \\
NGC 708 & 2.90 & 40.14 & 3.2 & 14.69 & 0.22 \\
NGC 1052 & 57.0 & 40.37 & 5.5 & 1.61 & 3.40 \\
NGC 2974 & 17.0 & 40.13 & 3.1 & 1.79 & 1.73 \\
NGC 4546 & 9.50 & 39.26 & 0.4 & 0.21 & 1.90 \\
NGC 5846 & 21.0 & 40.10 & 2.9 & 1.07 & 2.71 \\
NGC 5866 & 110.00 & 40.29 & 4.6 & 2.57 & 1.79 \\
NGC 5898 & 32.70 & 40.57 & 8.7 & 1.57 & 5.54 \\
NGC 6868 & 14.00 & 40.39 & 5.8 & 4.23 & 1.37 \\
NGC 7014 & 7.00 & 40.58 & 8.8 & 7.80 & 1.13 \\
NGC 7332 & 54.00 & 40.39 & 5.7 & 3.75 & 1.52
\end{tabular}

\subsubsection{NGC 4546}

This galaxy is classified as an SB0 object. Galletta (1987) and Bettoni et al. (1991) put in evidence ionized gas in this object counter rotating with respect to the stars. We confirm this counter rotation and put constraints on the shape of this object. Viewing angles show that gaseous disks can lie in the $Z Y$ plane.

\subsubsection{NGC 5846}

This is an E0 galaxy in the G50 group with a companion NGC 5846A $1^{\prime}$ to the south. The presence of atomic gas in that galaxy is not obvious, Roberts et al. (1991) only give a superior limit of $\mathrm{HI}$ gas mass $\left(M_{\mathrm{HI}}=510^{5} M_{\odot}\right)$. A strong X-ray emission was detected with Einstein satellite (Forman et al. 1985). Demoulin et al. (1984) have studied the ionized gas component using numerical subtraction technique and results obtained with FP observation are in agreement with this study. The line of sight velocity diagram shows a non axisymetric rotation, the North West $\left(200 \mathrm{~km} \mathrm{~s}^{-1}\right)$ part has a velocity amplitude greater than the South East part $\left(80 \mathrm{~km} \mathrm{~s}^{-1}\right)$. Viewing angles diagram shows that both Prolate and Oblate possibilities are possible.

\subsubsection{NGC 5866}

The main characteristic of this SO galaxy is his strong dust lane along the major axis. There are two companions, NGC 5907 and NGC 5879. This object presents a multiphase ISM: HI was detected by Haynes et al. (1990) but only a superior limit was given for HI mass. X-ray emission has been observed (Roberts et al. 1991; Pellegrini 1994) with an extension of $7.7^{\prime}$ along the major axis. Ionized gas has been found in that galaxy and the monochromatic map shows an extension of about $40^{\prime \prime}$ with a major axis almost aligned with respect to the stars. The line of sight velocity diagram shows a non axisymetric motion. The NW part has a velocity of $-200 \mathrm{~km} \mathrm{~s}^{-1}$ lower than the systemic velocity. $\phi$ viewing angle is not constrained. For this object too we have an $\mathrm{H}_{\alpha} /[\mathrm{NII}]$ map. The mean value 
on the ratio is 0.6 . In that case too the ratio is not constant but the gradient is lower than in NGC 404.

\subsubsection{NGC 5898}

This galaxy is an E0 object with a companion (NGC 5903) $5^{\prime}$ to the East. Only superior limits are given for $\mathrm{HI}$ and $\mathrm{X}$-ray gas masses. Stars are in counter rotation with respect to the ionized gas as shown by Bertola \& Bettoni (1988). In that case too, $\phi$ possible values region is wide $\left(60^{\circ}\right)$, and it is difficult to determine the global shape of the galaxy.

\subsubsection{NGC 6868}

This galaxy is classified as an E3 galaxy in the RC3 catalog and as an E2 in the RSA catalog. It belongs to the GR28 group with four other galaxies (Maia et al. 1989). Cold ISM is present with HI gas, but Roberts et al. (1991) give only a superior limit for the HI gas mass. NGC 6868 is the host galaxy of a radio source PKS 2005-489 (Savage et al. 1977). It was also detected with IRAS satellite in $60 \mu \mathrm{m}$ and $100 \mu \mathrm{m}$. Ionized gas has been put in evidence in spectroscopy by Phillips et al. (1986) and with CCD imaging by Hansen et al. (1991), Buson et al. (1993) and Macchetto et al. (1996). The line of sight velocity diagram shows a flat curve with a velocity amplitude of $\pm 150 \mathrm{~km} \mathrm{~s}^{-1}$. Possible viewing angles diagram show us that the more probable location plane of the gaseous disk is the $X Y$ plane (perpendicular to the short axis). The $\phi$ angle is more constrained with this possibility $\left(5^{\circ}<\phi<15^{\circ}\right)$. Possible axis ratios are also shown.

\subsubsection{NGC 7014}

It is classified as an SO object in the RC3 catalog. A companion is present $7^{\prime}$ to the west. No informations are available in radio or X-rays domain, only dust has been put in evidence (Sparks et al. 1985), ionized gas has been observed by spectroscopically by Phillips et al. (1986). The monochromatic map shows a complex structure with a strong gradient. The line of sight velocity diagram shows a non axisymetric motion, the NE branch has a velocity amplitude higher than the SW branch. We also notice that in the SW direction beyond $10^{\prime \prime}$, velocities are much spread out. On the geometry point of view, it seems that the Oblate possibility is the more probable with $20^{\circ}<\phi<60^{\circ}$ and $54^{\circ}<\theta<58^{\circ}$.

\subsubsection{NGC 7332}

This object is classified as an SO galaxy in the RC3 catalog. We have observed a complex system of two gaseous components. A detailed study is presented by Plana \& Boulesteix (1996).

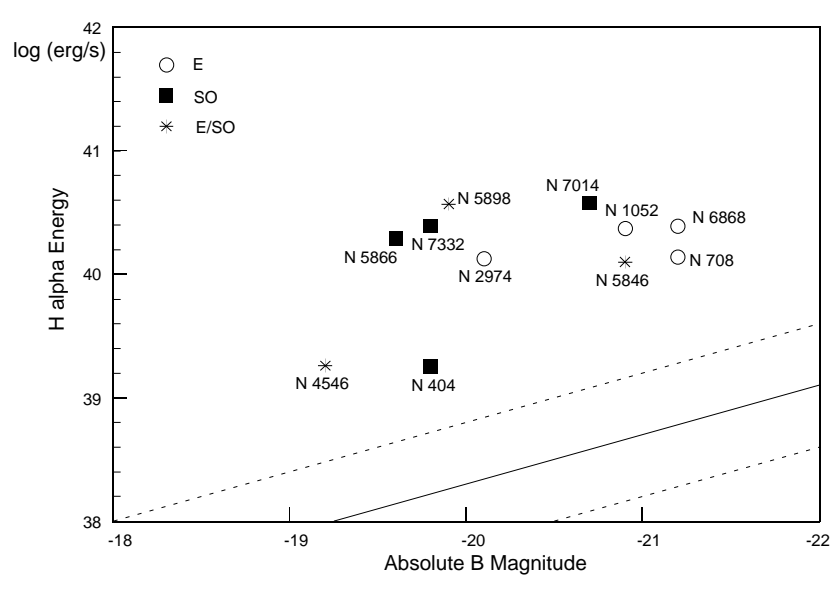

Fig. 45. $\mathrm{H}_{\alpha}$ luminosity versus $B$ absolute magnitude. The dashed lines represent Phillips et al. (1986) sample

\section{Global analysis}

\subsection{Photometry}

In this section we have tried to get an overall idea of the sample. Informations given previously allow us to investigate the spatial gas distribution and ionized gas mass/total mass ratio. We first look at $\mathrm{H}_{\alpha}$ luminosity repartition with respect to the $\mathrm{B}$ absolute magnitude. This is shown in Fig. $45 \mathrm{H}_{\alpha}$ has been derived from monochromatic maps and using $\mathrm{H}_{\alpha} /[\mathrm{NII}]$ ratio found on literature (Phillips et al. 1986; Goudfrooij et al. 1994). It appears that $\mathrm{H}_{\alpha}$ luminosity is 10 to 100 times more important than Phillips et al (1986) sample of emission line ellipticals detected by spectroscopy, which is consistent with Buson et al. (1993) study.

Monochromatic maps give us the gas distribution along the major axis. This extension varies considerably for the different galaxies. Figure 46 shows the radial surface brightness of the 11 galaxies for the gas and stars. The extension is different and the slope is different too, between the gas and the stars and between galaxies. In order to compare the gas surface brightness slope, an intensity gradient index has been introduced. It is derived from the intensity profile with as $I=f\left(r^{-n}\right)$ with $\mathrm{n}$ in the range $1-2$. Figure 47 shows the normalized gas extension ([NII] $6584 \AA$ ) versus the intensity gradient index. We can see that for a given gradient index, SO galaxies seem to have a larger gas extension.

\subsection{Geometry}

The geometrical aspect is the other major point of our study. Table 6 summarizes possible viewing angles $(\theta$ and $\phi)$ and possible axis ratios $(I / L$ and $S / L)$ derived from velocity fields and using de Zeeuw \& Franx model (1989). Our sample can be divided in 3 parts for the geometrical point of view. 


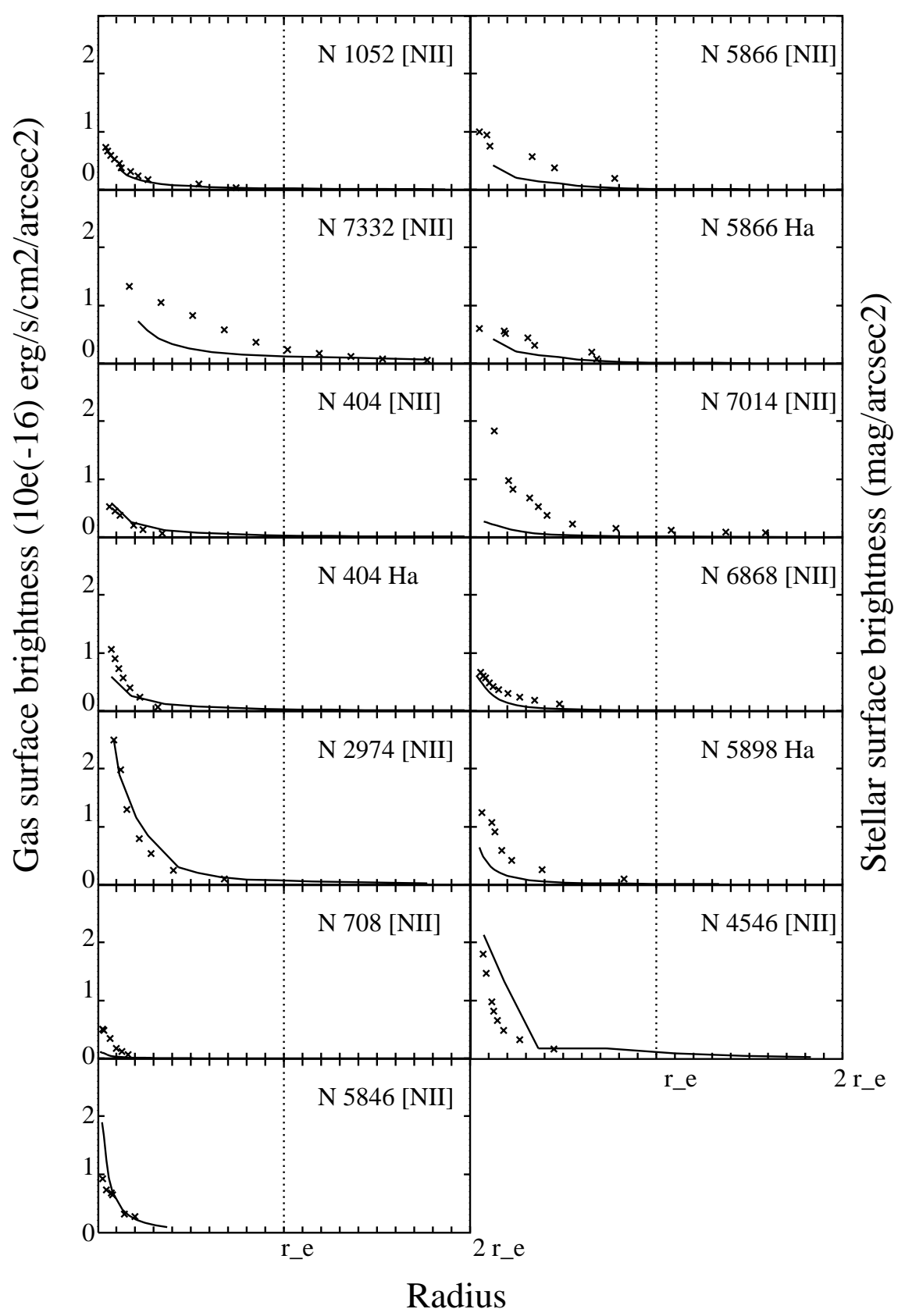

Fig. 46. Surface brightness of the ionized gas and the stars. The vertical dashed line represent the effective radius. Cross represent the gas brightness and continue line is the stellar brightness

- Galaxies with 2 gas components: NGC 708, NGC 1052 and NGC 7332.

NGC 708 shows a component almost aligned with the stars and another strongly decoupled. For the first component $\phi$ angle is not strongly constrained but it is enough to think that the gas disk is lying on the $Z Y$ plane (perpendicular to the long axis). For the second component the $\phi$ angle is not constrained at all and only the prolate shape seems to be possible. In that condition it is difficult to give gas disks location.

NGC 1052 and NGC 7332 cases have been discussed in a previous paper (Plana \& Boulesteix 1996). To summarize, component 1 of NGC 1052 have high probability to be located on $X Y$ plane but it is doubtly to have the second component on $Z Y$ plane, because of the none common values of axis ratios for that hypothesis. Situation for NGC 7332 is quite the same with simple 
way to find both components in the two perpendicular planes.

- Galaxies with no constrained $\phi$ angle: NGC 404, NGC 2974, NGC 5866 and NGC 5898.

For that objects, the gas velocity field MA is almost aligned with the stellar MA. The consequence is a wide region allowed for the $\phi$ angle.

- Galaxies with good constraint on $\phi$ angle: NGC 4546, NGC 5846, NGC 6868 and NGC 7014.

In that case several possibilities are encountered:

Good $\phi$ determination and one possibility for the gas location: NGC $4546 \phi=57 \pm 5$ in a Prolate case.

Good $\phi$ determination but two possibilities for the gas location: NGC $5846 \phi=-22 \pm 15$ in a Prolate case and $\phi=67^{\circ} \pm 12^{\circ}$ in a Oblate case.

Good $\phi$ determination, two possibilities for gas location but one more probable than the other:

NGC $6868 \phi=12^{\circ} \pm 5^{\circ}$ in a Prolate case.

NGC $7014 \phi=5^{\circ} \pm 3^{\circ}$ in a Prolate case.

As we can see, the $\phi$ viewing angle determination is strongly dependant of the misalignment between gas and stars MA.

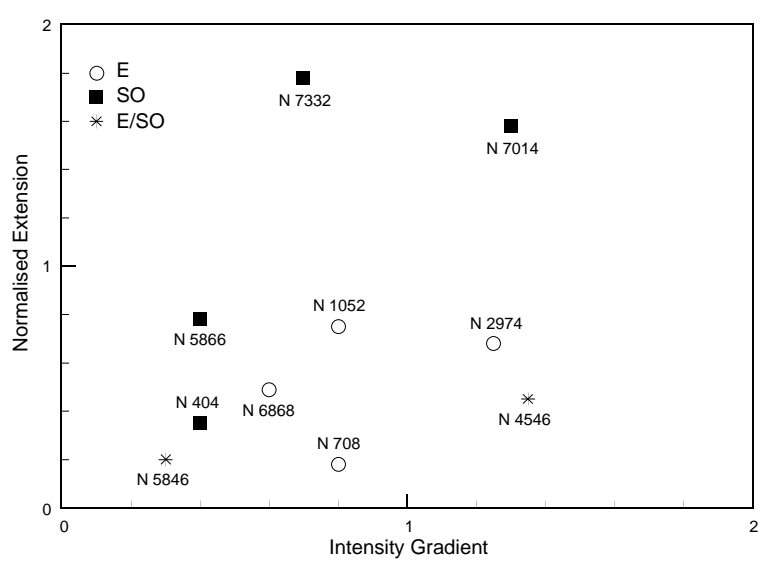

Fig. 47. Normalized gas extension (in unit of $r_{\mathrm{e}}$ ) versus the intensity gradient index

\section{Conclusion}

This paper presents scanning Perot-Fabry observations of ionized gas in 11 elliptical and lenticular galaxies. We show monochromatic and velocity maps for each object. Using the model developed by de Zeeuw and Franx in 1989, we could give informations about the geometrical aspect of the triaxial ellipsoid such as viewing angles and axis ratios. But this calculation gives interesting results (narrow possible viewing angles regions) only if gas and stars major axis are not aligned, if not regions are too wide to give any valuable information. Three galaxies of our sample show a
Table 6. Viewing angles: $\theta, \phi$ and axis ratios $I / L, S / L$. For each galaxy we give the both possibilities Oblate or Prolate when it is possible. Note: the $\theta$ angle is the same as the inclination angle deduced from velocity map in Table 4

\begin{tabular}{|c|c|c|c|c|}
\hline Name & Viewin & g Angles & Axis $F$ & Ratios \\
\hline & & $\phi$ & $I / L$ & $S / L$ \\
\hline NGC 404 & & & & \\
\hline Prolate: & $65^{\circ} \pm 3^{\circ}$ & $35^{\circ} \pm 25^{\circ}$ & $0.75 \pm 0.25$ & $0.65 \pm 0.15$ \\
\hline Oblate: & $65^{\circ} \pm 3^{\circ}$ & $85^{\circ} \pm 5^{\circ}$ & - & - \\
\hline NGC 708 & & & & \\
\hline Compo \#1 & & & & \\
\hline Prolate: & - & - & - & - \\
\hline Oblate: & $45^{\circ} \pm 5^{\circ}$ & $-35^{\circ} \pm 25^{\circ}$ & $0.75 \pm 0.25$ & $0.45 \pm 0.5$ \\
\hline Compo \#2 & & & & \\
\hline $\begin{array}{l}\text { Prolate: } \\
\text { Oblate: }\end{array}$ & $\begin{array}{c}55^{\circ} \pm 5^{\circ} \\
-\end{array}$ & $\begin{array}{c}-55^{\circ} \pm 35^{\circ} \\
-\end{array}$ & $\begin{aligned} & 0.77 \pm 0.17 \\
&-\end{aligned}$ & $\begin{array}{c}0.45 \pm 0.5 \\
-\end{array}$ \\
\hline NGC 1052 & & & & \\
\hline Compo \#1 & & & & \\
\hline Prolate: & & $13^{\circ} \pm 3^{\circ}$ & $0.55 \pm 0.05$ & $0.50 \pm 0.15$ \\
\hline Oblate: & $47^{\circ} \pm 5^{\circ}$ & $48^{\circ} \pm 20^{\circ}$ & $0.80 \pm 0.20$ & $0.52 \pm 0.17$ \\
\hline Compo \#2 & & & & \\
\hline Prolate: & $54^{\circ} \pm 5^{\circ}$ & $3^{\circ} \pm 1^{\circ}$ & $0.62 \pm 0.15$ & $0.45 \pm 0.15$ \\
\hline Oblate: & $54^{\circ} \pm 5^{\circ}$ & $45^{\circ} \pm 35^{\circ}$ & $0.90 \pm 0.10$ & $0.70 \pm 0.05$ \\
\hline NGC 2974 & & & & \\
\hline $\begin{array}{l}\text { Prolate: } \\
\text { Oblate: }\end{array}$ & $60^{\circ} \pm 5^{\circ}$ & $-45^{\circ} \pm 25^{\circ}$ & $0.75 \pm 0.15$ & $0.45 \pm 0.15$ \\
\hline Oblate: & & & - & - \\
\hline $\begin{array}{l}\text { NGC } 4546 \\
\text { Prolate: }\end{array}$ & & & & \\
\hline $\begin{array}{l}\text { Prolate: } \\
\text { Oblate: }\end{array}$ & $\begin{array}{c}55^{\circ} \pm 3^{\circ} \\
-\end{array}$ & $\begin{array}{c}57^{\circ} \pm 5^{\circ} \\
-\end{array}$ & $\begin{array}{c}0.57 \pm 0.07 \\
-\end{array}$ & $\begin{array}{c}0.38 \pm 0.10 \\
-\end{array}$ \\
\hline NGC 5846 & & & & \\
\hline Prolate: & $49^{\circ} \pm 5^{\circ}$ & $-22^{\circ} \pm 15^{\circ}$ & $0.74 \pm 0.12$ & $0.60 \pm 0.3$ \\
\hline Oblate: & $49^{\circ} \pm 5^{\circ}$ & $-67^{\circ} \pm 12^{\circ}$ & $0.85 \pm 0.15$ & $0.60 \pm 0.3$ \\
\hline $\begin{array}{l}\text { NGC } 5866 \\
\text { Prolate: }\end{array}$ & & & & \\
\hline $\begin{array}{l}\text { Prolate: } \\
\text { Oblate: }\end{array}$ & $58^{\circ} \pm 3^{\circ}$ & $45^{\circ} \pm 45^{\circ}$ & $0.79 \pm 0.17$ & $0.45 \pm 0.15$ \\
\hline Dlate: & & & - & - \\
\hline $\begin{array}{l}\text { NGC } 5898 \\
\text { Prolate. }\end{array}$ & & & & \\
\hline $\begin{array}{l}\text { Prolate: } \\
\text { Oblate: }\end{array}$ & $\begin{array}{c}58^{\circ} \pm 3^{\circ} \\
-\end{array}$ & $\begin{array}{c}-30^{\circ} \pm 30^{\circ} \\
-\end{array}$ & $\begin{array}{c}0.70 \pm 0.20 \\
-\end{array}$ & $\begin{array}{c}0.60 \pm 0.3 \\
-\end{array}$ \\
\hline NGC 6868 & & & & \\
\hline Prolate: & $52^{\circ} \pm 5^{\circ}$ & $12^{\circ} \pm 5^{\circ}$ & $0.65 \pm 0.19$ & $0.55 \pm 0.22$ \\
\hline Oblate: & $52^{\circ} \pm 5^{\circ}$ & $55^{\circ} \pm 22^{\circ}$ & $0.84 \pm 0.17$ & $0.57 \pm 0.27$ \\
\hline NGC 7014 & & & & \\
\hline Prolate: & $56^{\circ} \pm 2^{\circ}$ & $5^{\circ} \pm 2^{\circ}$ & $0.47 \pm 0.03$ & $0.40 \pm 0.05$ \\
\hline Oblate: & $56^{\circ} \pm 2^{\circ}$ & $37^{\circ} \pm 22^{\circ}$ & $0.82 \pm 0.17$ & $0.47 \pm 0.17$ \\
\hline NGC 7332 & & & & \\
\hline $\begin{array}{l}\text { Compo \#1 } \\
\text { Prolate: }\end{array}$ & & & & \\
\hline $\begin{array}{l}\text { Prolate: } \\
\text { Oblate: }\end{array}$ & $56^{\circ} \pm 10^{\circ}$ & $60^{\circ} \pm 10^{\circ}$ & $0.35 \pm 0.1$ & $0.15 \pm 0.1$ \\
\hline Compo \#2 & & & & \\
\hline $\begin{array}{l}\text { Prolate: } \\
\text { Oblate: }\end{array}$ & $\begin{array}{c}67^{\circ} \pm 10^{\circ} \\
-\end{array}$ & $\begin{array}{c}42.5^{\circ} \pm 17^{\circ} \\
-\end{array}$ & $\begin{array}{c}0.30 \pm 0.1 \\
-\end{array}$ & $\begin{array}{c}0.12 \pm 0.07 \\
-\end{array}$ \\
\hline
\end{tabular}


complex system of double gaseous components: NGC 708, NGC 1052 and NGC 7332. The presence of such multiple components addresses the question of gas origin and fate. This implies an external origin of the gas, at least for a part of the gas content. One scenario is to imagine that the galaxy had several encounters during his life. The case of NGC 708 is certainly the most interesting, because one component is aligned with the stars and the other is decoupled. In that case the cooling flow hypothesis can not explain the presence of such gaseous components. On another hand two objects, NGC 404 and NGC 2974 show a perfect agreement between gas and stars which can be candidates for internal origin.

Monochromatic maps give us the possibility to have ionized gas masses. The range of the masses is between $410^{3} M_{\odot}$ and $910^{4} M_{\odot}$, which is few in comparison with the total mass of the galaxy estimated from the maximal gas velocity (cf. Table 5). As Buson et al. (1993) analysis, our sample shows that $\mathrm{H}_{\alpha}$ luminosity is 10 to 100 times larger than the spectroscopic survey from Phillips et al. (1986). It can mean that galaxies gas content is simply larger or that a higher fraction of gas has been ionized. The gas distribution is also quite different between galaxies. In a forcoming paper we will discuss more deeply the interpretation we can give for galaxy shape and orientation of elliptical galaxies.

Acknowledgements. We wish to thank the Local Allocation Time Committee of the Special Astrophysical Observatory (Russia) as well as Drs. V. Afanasiev, A. Burenkov, S. Dodonov, V. Vlasiuk and Mr S. Drabek who helped us efficiently and built partially a local CIGALE instrument indispensable for the observations. We also want to thank M. Marcelin for his help in the writing of this article.

\section{References}

Afanasiev V.L., Balega Y.Y., Grudzinsky M.A., et al., 1987, in "Tekhnika sredstv svyazi", Ser. Teknika televideniya 5, 13 (in russian)

Amram P., Boulesteix J., Georgelin Y.P., et al., 1991, "The Messenger" (ESO) 64, 44

Baars J., Wendkel H., 1976, A\&A 48, 405

Barbon R., Capacioli M., Rampazzo R., 1982, A\&A 115, 388

Bertola F., Bettoni D., 1988, ApJ 329, 102

Bertola F., Bettoni D., Danziger J., et al., 1991, ApJ 373, 369

Bettoni D., Galletta G., Osterloo T., 1991, MNRAS 248, 544

Bettoni D., 1992, A\&AS 96, 333

Boulesteix J., Georgelin Y., Marcelin M., Monnet G., 1983, SPIE Conf. Instr. Astron. V 445, 37
Boulesteix J., 1993, "ADHOC reference manual", Publications de l'Observatoire de Marseille

Braine J., Dupraz C., 1994, A\&A 283, 407

Buson L.M., Sadler E.M., Zeilinger W.W., et al., 1993, A\&A 280, 409

Davies R.L., Illingworth G.D., 1986, ApJ 302, 234

Demoulin-Ulrich M.H., Butcher H.R., Boksenberg A., 1984, ApJ 285, 527

Fabbiano G., Kim D.W., Trinchieri G., 1992, ApJS 80, 531

Faber S.M., Wegner G., Burstein D., et al., 1989, ApJS 69, 763

Fanti C., Fanti R., Ciota I., Parma C., 1977, A\&AS 29, 279

Forman W., Jones C., Tucker W., 1985, ApJ 293, 102

Galletta G., 1987, ApJ 318, 531

van Gorkom J.H., Knapp G.R., Raimond E., Faber S.M., Gallager J.S., 1986, AJ 91, 791

Goudfrooij P., Hansen L., Jorgensen H.E., Norgaard-Nielsen H.U., 1994, A\&AS 105, 341

Hansen L., Jorgensen H.E., Norgaard-Nielsen H.U., 1991, AJ 243, 49

Haynes M., Herter T., Barton A., Benensohn J., 1990, AJ 99, 1740

Heckman T., Baum S., van Breugel W., McCarthy, 1989, ApJ 338,48

Heiligman, Scharzschild M., 1979, ApJ 233, 872

Kim D.W., Guhathakurta P., van Gorkom J., Jura M., Knapp G., 1988, ApJ 330, 684

Kim D.W., 1989, ApJ 346, 653

Knapp G.R., Turner E.L., Cunniffe P.E., 1985, AJ 90, 454

Laval A., Boulesteix J., Georgelin Y.P., Georgelin Y.M., Marcelin M., 1987, A\&A 175, 199

Landsdale Cataloged galaxies and QSO observed in the IRAS survey 1989

Lees J.F., Knapp G.R., Rupen M.P., Phillips T.G., 1991, ApJ 379,177

Lequeux J., 1983, A\&A 125, 394

Macchetto F., Pastoriza M., Caon N., et al., 1996, A\&AS 120, 463

Maia M., Da Costa L., Latham D., 1989, ApJS 69, 809

Osterbrock D.E., 1974, Astrophysics of Gaseous Nebulae. W.A. Freeman and Company, San Francisco, p. 71

Pellegrini S., 1994, A\&A 292, 395

Phillips M., Jenkins C., Dopita M., Sadler E., Binette L., 1986, AJ 91, 1062

Plana H., Boulesteix J., 1996, A\&A 307, 391

Roberts M.S., Hogg D.E., Bregman J.N., Forman W.R., Jones C., 1991, ApJS 75, 751

Savage A., Bolton J., Wright A., 1977, MNRAS 179, 135

Sparks W., Wall J., Thorne D., et al., 1985, MNRAS 217, 87

Tohline J.E., Simonson G.F., Caldwell N., 1982, ApJ 252, 92

Wiklind T., Henkel C., 1990, A\&A 239, 125

de Zeeuw T., Franx M., 1989, ApJ 343, 617 